\title{
Butterflies of the Garo Hills of Meghalaya, northeastern India: their diversity and conservation
}

\author{
Krushnamegh Kunte ${ }^{1}$, Sanjay Sondhi ${ }^{2}$, Bensen M. Sangma ${ }^{3}$, Rohan Lovalekar ${ }^{4}$, \\ Kedar Tokekar ${ }^{5}$ \& Gaurav Agavekar ${ }^{6}$ \\ 1,2,4,5,6 Indian Foundation for Butterflies. No. 9, Snehanagar, Amruthahalli Road, Byatarayanapura, Bengaluru, Karnataka 560092, \\ India \\ 1,6 National Center for Biological Sciences (NCBS), GKVK, Bellary Road, Bengaluru, Karnataka 560065, India \\ 2 Titli Trust. 49 Rajpur Road Enclave, Dhoran Khas, near IT Park, P.O. Gujrada, Dehradun, Uttarakhand 248001, India \\ ${ }^{3}$ Samrakshan Trust, Bolsalgre, Baghmara, Meghalaya 794102, India \\ Email: ${ }^{1}$ krushnamegh@ifoundbutterflies.org, ${ }^{2}$ sanjay.sondhi1@gmail.com, ${ }^{3}$ bensen@ifoundbutterflies.org, ${ }^{4}$ rohan@ifoundbutterflies. \\ org, ${ }^{5}$ kedar@ifoundbutterflies.org, ${ }^{6}$ gaurav@ifoundbutterflies.org
}

Date of publication (online): 12 September 2012 Date of publication (print): 12 September 2012 ISSN 0974-7907 (online) | 0974-7893 (print)

Editor: Tim New

Manuscript details:

Ms \# 02945

Received 14 November 2011

Final received 11 April 2012

Finally accepted 24 June 2012

Citation: Kunte, K., S. Sondhi, B.M. Sangma, R. Lovalekar, K. Tokekar \& G. Agavekar (2012). Butterflies of the Garo Hills of Meghalaya, northeastern India: their diversity and conservation. Journal of Threatened Taxa 4(10): 2933-2992.

Copyright: (c) Krushnamegh Kunte, Sanjay Sondhi, Bensen M. Sangma, Rohan Lovalekar, Kedar Tokekar \& Gaurav Agavekar 2012 Creative Commons Attribution 3.0 Unported License. JoTT allows unrestricted use of this article in any medium for non-profit purposes reproduction and distribution by providing adequate credit to the authors and the source of publication.

Author Contributions, Author Details and Acknowledgments: see end of this article.
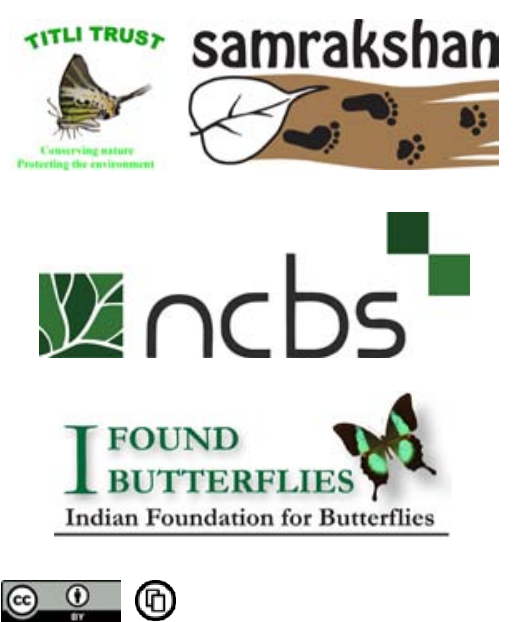

OPEN ACGESS | FREE DOWNLOAD
Abstract: The Garo Hills of Meghalaya, northeastern India, form the westernmost boundary of the globally recognized Indo-Myanmar Biodiversity Hotspot. The butterfly fauna of the Garo Hills is expected to be diverse, but it has not been properly sampled before. We surveyed butterflies in Balpakram National Park, Baghmara Reserve Forest and Siju Wildlife Sanctuary in southern Garo Hills, and Nokrek National Park in Western Garo Hills during four visits amounting to 49 days spread over two seasons, pre-monsoon (April-May) and post-monsoon (November-December), between 2008 and 2010. Here we report 298 butterfly species for the Garo Hills, eight of which are legally protected under Schedule I and 33 under Schedule II of the Indian Wildlife (Protection) Act, 1972. Our species accumulation curve suggests that many species remain to be discovered in the Garo Hills, and we expect the total species richness to be closer to $600-650$ species. Little quantitative information exists on populations and seasonal occurrence of butterflies in India. Therefore, we recorded the number of individuals of each species in one or three hour counts during our surveys, and here we report season-wise relative abundances of 298 species from 3,736 individuals. We also report significant range extensions of two Schedule I species: Elymnias peali and Prothoe franck regalis, from the Garo Hills. These findings underscore the significance of the Garo Hills for butterfly conservation in India, and our work forms a baseline for future quantitative work on the diversity and conservation of butterflies in this biodiversity hotspot.

Keywords: Butterfly species diversity, coal mining, conservation planning; IndoBurmese Biodiversity Hotspot, Lepidoptera, participatory management.

Abstract in Garo language: Garo Hills ian Northeastern India-ni salirambatsranggipa bako ga.aka, jekon a.gilsak gimiko Indo-Myanmar Biodiversity hotspot ba a.gilsak gimikchin rokomari jo.ong guk, do.omat aro sambolrangni gnanganina mingsinga. la A.chik A.song ba Garo Hills-o bang.bea rokomni Mepliprang donga ine sakantian chanchichipa indiba iani bidingo darangba namedake sandirikite separakaniko da.alona kingkingba dakkujachim. Chinga South Garo Hills-ni Balpakram National Park, Baghmara Reserve Forest aro Siju Wildlife Sanctuary unbaksana West Garo Hills-oniko Nokrek National Park ia biaprangoniko Mepliprangni rokomko sandirikite niaha. Je sandirikite nianiko chinga chingni changbri songrebaani somoirango dakangaha, jeon chinga sal 49 ni gisepo dakaha. Chinga 2008 aro 2010 bilsirangni gisepo changbri dake re.baaha mongsongbate chinga Wachi karina skang (April-May) aro Wachi karini jaman (November-December) ia jarango sandie nianiko dakaha. la sandie nianio chinga, 298 rokomni Mepliprangko sandie man.aha, jeoni rokom chet-de Wildlife Protection Act1972 ni gita Schedule I aro rokom 33 Schedule II species-rang ong.a. Chingni sandie man.ana agreba Garo Hills-o nikkugijagipa dingtangdingtang rokomni Mepliprang dongkuenggen ineba chanchichipenga aro Garo Hills-o rokom 600 oni rokom 650 Mepliprang dongchongmotna nanga ine chinga ka.donga. India-o baita mancha bang.a uamangni jelanikoba adita see rakkianirangba donga, unigimin chinga rokomantini jelanikoba kontasa ba konta gittamni somoirango chane nianiko daka, jeon rokom 298 oniko chinga 3,736 mepliprangko chane man.aha. Una agreba chinga Garo Hills-o rokom gni Schedule I species-o ga.akgipa Mepliprang: Elymnias peali and Prothoe franck regalis ia rokomrangni mongsonggipa dongchakram bi.apkoba name manchaan ui.e ra.na gita man.aha. la chingni sandirikite man.aniara India gimiko Mepliprangko ripinganina ia Garo Hills-ba mongsonggipa bi.ap ong.a ine namen sakkiko on.a aro ia chingni kamrangara mikkangchi ia Biodiversity Hotspot-o mepliprang aro adok chigako nambate ripingangna namen dakchakbeani ong.gen ine chinga bebera.a. 


\section{INTRODUCTION}

The northeastern region of India, south of the Brahmaputra River, is part of the globally recognized Indo-Myanmar biodiversity hotspot and is host to a remarkable biodiversity that includes a high proportion of endemic, rare and endangered species (http://www.biodiversityhotspots.org/xp/hotspots/ indo_burma/Pages/default.aspx). The Garo Hills of the northeastern state of Meghalaya (previously part of Assam) form the north-westernmost limit of the Indo-Myanmar Biodiversity Hotspot. From there the hotspot extends southeastwards to cover the Khasi, Jaintia, Naga, Manipur and Mizo Hills (together encompassing the Patkai Hills) in northeastern India, and all of the Indo-Chinese subregion. The Indian part of this hotspot is one of the most species-rich regions in the Indian Subcontinent, with considerable endemism at subspecies level (Kunte in preparation). The high species richness and endemism make this an especially important region for butterfly diversity and conservation in India.

Early European lepidopterists extensively explored this biodiversity hotspot between 1840 and 1950 and described hundreds of butterfly species and subspecies (summarized in Moore 1890-1892, 1893-1896, 18961899, 1899-1900, 1901-1903, 1903-1905; Swinhoe 1905-1910, 1910-1911, 1911-1912, 1912-1913; Evans 1932; Parsons \& Cantlie 1948; Cantlie 1952, 1956). The major taxonomic and natural history work in this region was done in the Khasi and Jaintia Hills in eastern Meghalaya (Swinhoe 1893, 1896; Parsons \& Cantlie 1948; Cantlie 1952, 1956), in Cachar Hills in southern Assam and Bangladesh (Butler 1879), and in the Naga-Manipur Hills (Tytler 1911, 1912, 1914, 1915a,b, 1926a,b). The pace of species discovery and accumulation of bionomic information on butterflies of this region has subsequently slowed down as the region has received less attention from lepidopterists with a few notable exceptions (Radhakrishnan et al. 1989; Larsen 2004; Kunte 2009, 2010). Nevertheless, some areas have historically remained practically unexplored.

As far as we know, one area that has never been properly surveyed is the Garo Hills in western Meghalaya. The Garo Hills are loosely connected in the east with the Khasi and Jaintia Hills in eastern Meghalaya surrounding the Shillong Plateau and
Cherrapunji (Image 1). As mentioned above, the Khasi and Jaintia Hills were intensively surveyed by early European lepidopterists, but those hills have been largely denuded due to coal and limestone mining and agriculture in the past 150 years. Therefore, little forest and associated biodiversity are now to be found in those hills. On the other hand, the Garo Hills still harbor substantial swaths of evergreen and semi-evergreen forests (Forest Survey of India 2009) and support a considerable proportion of endangered species such as the Asian Elephant, Bengal Tiger, Hoolock Gibbon and Serow. A total of at least 85 mammal, 23 amphibian, 33 reptile and 270 bird species have so far been reported from the Garo Hills (Kamal Medhi, pers. comm., 2011). However, butterflies in this landscape are poorly known due to lack of surveys. We assume that some information remains unpublished but available on specimen labels in various research collections and museums worldwide. This should be especially true of the Natural History Museum, London, and the National Zoological Collection of Zoological Survey of India, Kolkata, where most of the collections by early British lepidopterists are currently situated. We hope that this information will slowly become available as we continue our work in these museums. Nonetheless, little is known about current populations, distributions, seasonality and occurrence of butterflies of the Garo Hills, and of the entire northeastern India in general. This information is important for naturalists and ecologists as well as for policy-makers and conservationists. With this in mind, in the past few years we have been surveying butterfly populations in several northeastern Indian states to fill gaps in our current knowledge of Indian butterflies. As part of this long-term initiative, we have especially focused our attention on surveying the little-known butterfly diversity in the Garo Hills. The work presented below is the first outcome of our surveys in the Garo Hills. This and our subsequent work will generate baseline information on butterflies by intensively surveying populations across seasonal, altitudinal and habitat gradients. We hope that this will be useful in documenting the rich biodiversity of the Garo Hills. 


\section{MATERIAL AND METHODS}

\section{Study area}

The Garo Hills of western Meghalaya: The Garo Hills cover approximately a third of Meghalaya's total area of $22,429 \mathrm{~km}^{2}$. They are distributed among three administrative districts: the East Garo Hills, the South Garo Hills, and the West Garo Hills (Image 1). So far, we have surveyed butterflies only in the West and South Garo Hills Districts, where several important protected forest areas are situated. The West Garo Hills District is spread over $3,715 \mathrm{~km}^{2}$, out of which $2,717 \mathrm{~km}^{2}$, or approximately $73 \%$, is forested (Forest Survey of India 2009). More than half of this forest is highly disturbed and the rest is moderately disturbed (Forest Survey of India 2009). However, evergreen forests are still largely intact in the Nokrek National Park-Tura Peak areas (Image 1).

The South Garo Hills District covers an area of $1,849 \mathrm{~km}^{2}$, of which $1,689 \mathrm{~km}^{2}$, or approximately $91 \%$, is forested (Forest Survey of India 2009). However, one-third of this is highly degraded forest, and the rest is moderately disturbed by jhum or shifting cultivation (Forest Survey of India 2009). Good forest patches are still found in the Balpakram-Baghmara landscape (Image 1, and below), where we have done most of our work so far. A total of approximately $600 \mathrm{~km}^{2}$ (presently $64 \%$ of its total landmass) of evergreen and secondary semi-evergreen forest of the BalpakramBaghmara landscape is situated in four protected areas managed by the Meghalaya Forest Department: the Balpakram National Park $\left(220 \mathrm{~km}^{2}\right)$, Siju Wildlife Sanctuary $\left(6 \mathrm{~km}^{2}\right)$, Baghmara Reserve Forest $\left(44.29 \mathrm{~km}^{2}\right)$ and Rewak Reserve Forest $\left(\sim 4 \mathrm{~km}^{2}\right)$, the rest falls under 36 tribal community lands called Akings $\left(\sim 330 \mathrm{~km}^{2}\right)$ (Image 1). Thus, nearly one-third of the district's area is covered by forests of the BalpakramBaghmara landscape. Most of this forest is at low elevations (altitudinal range 150-875 m), and may be classified as the Cachar tropical evergreen forest, originally dominated by Palaquim spp., Diospyros topiosa, Dipterocarpus turbinus, Messua ferrea and other large evergreen trees (Champion \& Seth 1968). Almost all the forest patches now have few very large evergreen trees, and they have a variable proportion of deciduous trees and bamboo depending on the extent of human disturbance, such as the intensity and extent of jhum cultivation. These forest patches are still rich in tropical floral and faunal elements, and the region has one of the highest recorded densities of the Asian elephant in the world.

Surveyed localities and dates: KK, SS, BMS, RL and GA surveyed butterflies at several localities in the Baghmara Reserve Forest (henceforth 'Baghmara

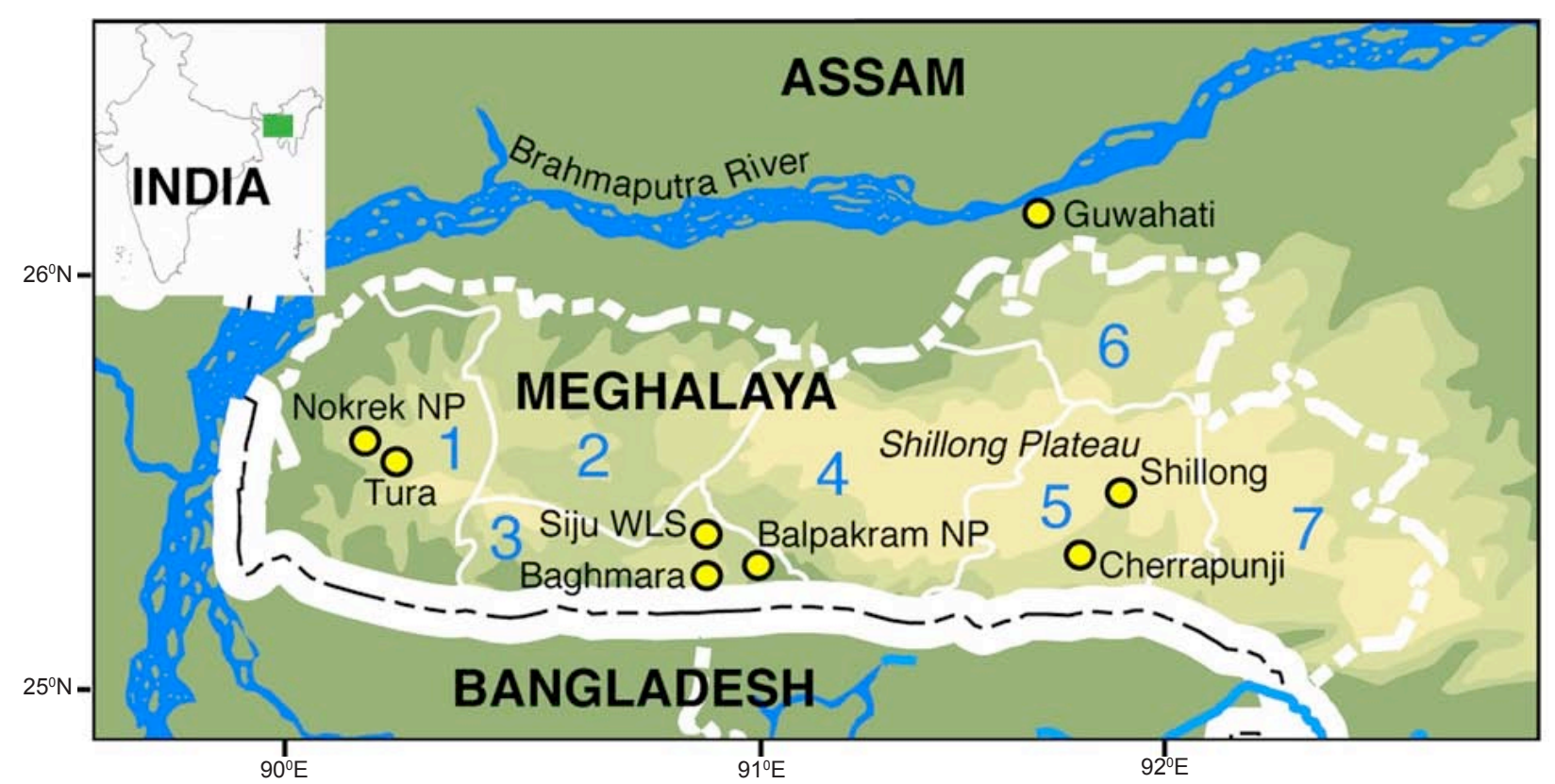

Image 1. Map of Meghalaya showing the locations of Baghmara, Balpakram NP, Siju WLS, and Nokrek NP in the Garo Hills. Also shown are major towns in the region, and district boundaries (thin white lines). The districts of Meghalaya are as follows: 1. West Garo Hills, 2. East Garo Hills, 3. South Garo Hills, 4. West Khasi Hills, 5. East Khasi Hills, 6. Ri Bhoi, 7. Jaintia Hills. 
RF'), the Siju Wildlife Sanctuary (henceforth 'Siju WS'), and outskirts of the Balpakram National Park (henceforth 'Balpakram NP') in the vicinity of Gongrot, Taidang, and Halwa Atong akings (akings are Garo tribal villages with the associated community lands). KT surveyed butterflies at the Nokrek National Park (henceforth 'Nokrek NP') and on the Balpakram Plateau within the Balpakram NP. We made four field trips to these areas to survey butterflies, on which we spent 53 days in the field and collected quantitative data on 49 days at the following specific localities (all dates in this paper follow the format, yyyy $/ \mathrm{mm} / \mathrm{dd}$ ):

Baghmara RF: (1) Karwani, Bhawanipur and Panda (streams in Baghmara RF near Baghmara Town): 2008/05/02,04; 2009/11/16, 21; 2009/12/0708, 13, 15, 17, 19-20; 2010/04/30; 2010/05/01-03, 07, 11, 13-14. (2) Simsang trail (footpath parallel to the Simsang River in Baghmara RF near Baghmara Town): 2008/05/02; 2009/12/11-12, 21.

Balpakram NP, and Gongrot-Halwa Atong area: (1) Taidang, Jidung and Rongrok (streams near Gongrot Village): 2008/05/03; 2009/11/16-19; 2009/12/05-06; 2010/05/04-06. (2) Rani Miksuram (stream near Halwa Atong Village): 2008/05/02; 2009/11/16; 2009/11/20. (3) Me Cheng (stream near Halwa Atong Village): 2009/11/20. (4) Balpakram Plateau: 2009/11/13-16; 2010/05/15-16.

Siju WS: 2009/11/22;2009/12/03-04;2010/05/08 09.

Nokrek NP: 2009/11/06-09. The latitudes and longitudes of the major localities were: (1) Baghmara town/RF: $25^{0} 12.543^{\prime} \mathrm{N} \& 90^{\circ} 38.011^{\prime} \mathrm{E}$; (2) Gongrot: $25^{\circ} 15.838^{\prime} \mathrm{N} \& 9^{\circ} 43.850^{\prime} \mathrm{E}$, (3) Balpakram Plateau: $25^{\circ} 15.876^{\prime} \mathrm{N} \& 9^{\circ} 51.837^{\prime} \mathrm{E},(4)$ Siju WS: $25^{\circ} 21.137^{\prime} \mathrm{N}$ \& 90041.187'E; and (5) Nokrek NP: $25^{\circ} 29.571^{\prime} \mathrm{N}$ \& $90^{\circ} 19.460^{\prime} \mathrm{E}$. In addition, KK also briefly surveyed rice fields and the Matcha Nokpante Community Reserve near the Samrakshan Trust's field office in Baghmara Town.

We sampled butterflies in several habitats at the above localities: evergreen forest streams, forested hill slopes, and neighborhoods of Gongrot and Halwa Atong villages. Neighborhoods of Gongrot and Halwa Atong included cashew and other orchards, abandoned jhums (areas of shifting cultivation) and extremely disturbed secondary forest, through which we had to walk for $1-2 \mathrm{~km}$ to reach excellent patches of evergreen forest on hill slopes and along streams.

\section{SAMPLING AND OTHER METHODS}

Our field work usually took place between 0800 and $1700 \mathrm{hr}$ every day. However, the sampling effort was uneven over various visits and by different observers. KK recorded each and every individual butterfly seen during his two visits, all of his observations having been broken down into one-hour butterfly counts from the beginning to the end of each day. Thus, his data were completely quantitative. SS counted butterflies over several three-hour counts but many of his sightings were outside these counts, so his observations were a mixture of quantitative sampling and all-out searches. BS, RL and GA did not quantify butterfly abundance; all of their records were based on photographs that were later identified by KK. KT usually recorded total numbers for every species seen. Quantitative data presented in Table 5 are summed over all the quantitative observations and all-out (opportunistic) searches by all the observers. For both types of data collection, we followed a fairly uniform method: we walked along forest paths and forest streams, where we have found butterfly abundance to be the highest over years of field work. We followed commonly used paths leading into the forests to survey butterflies in the vicinity of tribal villages. Once we entered forests, we walked either along these paths, waded through forest streams, or followed numerous elephant and other animal trails that criss-cross these forests. We recorded each and every butterfly species (and every individual in case of KK, SS and KT) that we saw perched overhead, on surrounding vegetation, or in flight at any distance from us. Each record was noted in field notebooks on the spot, and most species, including the commonest ones, were photographed in the field for reference. However, not all butterflies could be identified from only the upperside or underside as is usually seen in the field. Many species groups have distinctive characteristics on both wing surfaces that need to be checked closely, and it is not always possible to see these without capturing butterflies for a closer look. These characteristics included specific bands and spots, hair pencils, brands, etc. (the last two only in males), in genera such as Baoris, Pelopidas, Jamides, Arhopala, Rapala, Mycalesis and Euploea. Therefore, we obtained permission from local forest officials to catch butterflies to photograph both their wing surfaces for identification. We caught butterflies 
with a net, photographed them for relevant distinctive characteristics with digital cameras, and then released them immediately on the spot. If butterflies could not be identified to a species level without dissecting their male genitalia, we recorded only the genus names or the species group to which they belonged. Thus, several of our records were, e.g., for "Halpe spp.", "Potanthus spp.", "Mycalesis sp.", or "Neptis nata/soma group". We hope to obtain permission to collect specimens and dissect their genitalia to determine species in these genera and species groups in the future. Images 3-18 give photographic proof of our butterfly sightings in the Garo Hills so far. We had taken majority of these pictures at the sampling localities mentioned above. Only a small proportion of species photographs, marked in images $3-18$ by asterisks, were taken outside of the Garo Hills. We have included them to illustrate those species for anyone interested in butterflies. These should prove to be a particularly valuable reference for people conducting field work in the Garo Hills or elsewhere in northeastern India in the future.

We paid particular attention to mud-puddling spots where several species could be spotted (Image 2), and we spent considerable amount of time at these spots because there was a fairly high turnover of butterflies. Some butterfly species were more easily seen on specific food sources such as rotting crabs, which may not always be readily available. Therefore, we used rotting crabs as bait, which at times attracted over a hundred individual butterflies and dozens of species (Image 2).

The annotated checklist we present below is

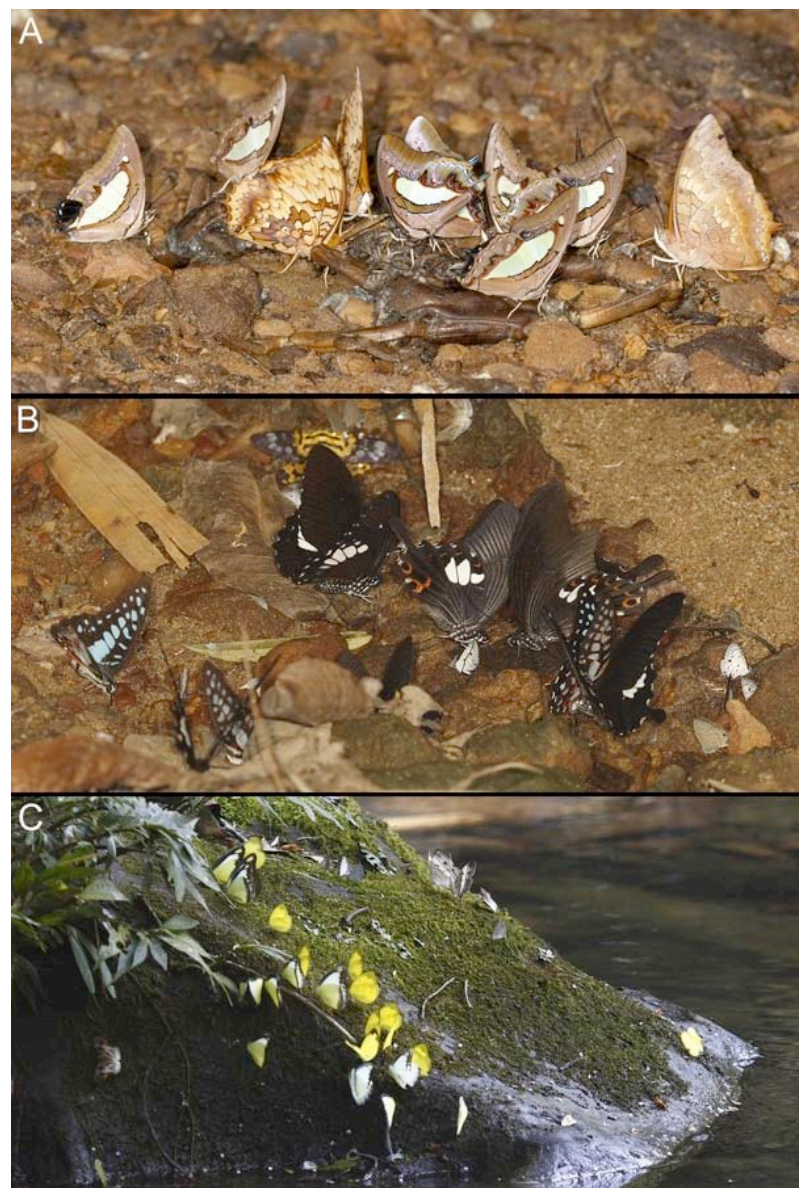

Image 2. Butterfly congregations at mud-puddling spots and on baits of rotting crabs. A - Charaxes congregation on rotting crabs used as bait along Taidang stream near Gongrot in Nov. 2009. B - A largely papilionid (Graphium and Papilio) and lycaenid (Megisba and Prosotas) mudpuddling congregation at Karwani stream in April 2008. C - A mixed congregation of pierids (Eurema and Appias), nymphalids (Cyrestis), lycaenids (Acytolepis) and hesperiids (Odontoptilum) at Taidang stream near Gongrot in April 2008. arranged alphabetically at every systematic level,

Table 1. Overview of taxonomic diversity of butterflies of the Garo Hills. Also shown is the taxonomic breakdown of butterflies protected under various schedules of the Indian Wildlife (Protection) Act, 1972 (WPA). Also see Table 4.

\begin{tabular}{|l|c|c|c|c|c|c|}
\hline \multirow{2}{*}{ Family } & \multirow{2}{*}{$\begin{array}{c}\text { Number of } \\
\text { subfamilies }\end{array}$} & \multirow{2}{*}{$\begin{array}{c}\text { Number of } \\
\text { genera }\end{array}$} & \multirow{2}{*}{$\begin{array}{c}\text { Number of } \\
\text { species }\end{array}$} & \multicolumn{3}{|c|}{ WPA-protected species } \\
\cline { 6 - 7 } & & & & Schedule I & Schedule II & Schedule IV \\
\hline Hesperiidae & $3(14 \%)$ & $34(22 \%)$ & $48(16 \%)$ & & 1 & 4 \\
\hline Lycaenidae & $5(23 \%)$ & $47(30 \%)$ & $72(24 \%)$ & 2 & 11 & 1 \\
\hline Nymphalidae & $10(45 \%)$ & $52(33 \%)$ & $121(41 \%)$ & 6 & 18 & 2 \\
\hline Papilionidae & $1(5 \%)$ & $8(5 \%)$ & $28(9 \%)$ & & 2 & \\
\hline Pieridae & $2(9 \%)$ & $12(8 \%)$ & $24(8 \%)$ & & 1 & \\
\hline Riodinidae & $1(5 \%)$ & $3(2 \%)$ & $5(2 \%)$ & & & \\
\hline Total: 6 & $\mathbf{2 2 ( 1 0 0 \% )}$ & $\mathbf{1 5 6}(\mathbf{1 0 0} \%)$ & $\mathbf{2 9 8}(\mathbf{1 0 0 \%})$ & $\mathbf{8}$ & 33 & $\mathbf{7}$ \\
\hline
\end{tabular}


Table 2. Subfamily-wise diversity of butterflies of the Garo Hills.

\begin{tabular}{|c|c|c|c|}
\hline Family & Subfamily & $\begin{array}{c}\text { Number of } \\
\text { genera }\end{array}$ & $\begin{array}{c}\text { Number of } \\
\text { species }\end{array}$ \\
\hline \multirow[t]{3}{*}{ Hesperiidae } & Coeliadinae & 5 & 6 \\
\hline & Hesperiinae & 23 & 32 \\
\hline & Pyrginae & 6 & 10 \\
\hline \multirow[t]{5}{*}{ Lycaenidae } & Curetinae & 1 & 2 \\
\hline & Lycaeninae & 1 & 2 \\
\hline & Miletinae & 3 & 3 \\
\hline & Polyommatinae & 23 & 36 \\
\hline & Theclinae & 19 & 29 \\
\hline \multirow[t]{10}{*}{ Nymphalidae } & Apaturinae & 4 & 4 \\
\hline & Biblidinae & 1 & 2 \\
\hline & Charaxinae & 2 & 9 \\
\hline & Cyrestinae & 2 & 3 \\
\hline & Danainae & 4 & 15 \\
\hline & Heliconiinae & 6 & 9 \\
\hline & Limenitidinae & 13 & 30 \\
\hline & Nymphalinae & 7 & 16 \\
\hline & Pseudergolinae & 2 & 2 \\
\hline & Satyrinae & 11 & 31 \\
\hline Papilionidae & Papilioninae & 8 & 28 \\
\hline \multirow[t]{2}{*}{ Pieridae } & Coliadinae & 4 & 7 \\
\hline & Pierinae & 8 & 17 \\
\hline Riodinidae & Nemeobiinae & 3 & 5 \\
\hline Total: 6 & 22 & 156 & 298 \\
\hline
\end{tabular}

from family and subfamily names to genera and species. Taxonomic information, including updated family, subfamily, genus, species and subspecies names, as well as English names of subspecies, was taken from the Butterflies of India website (http:// ifoundbutterflies.org/) (Kunte et al. 2011), which is itself based on an upcoming subspecies-level catalogue of Indian butterflies (Kunte in prep.). Note that parentheses have not been used for authors and years where genus assignments have changed, but the names that we have used should be easily traceable in standard taxonomic works. Further taxonomic details will be found elsewhere (Kunte in prep.).

\section{RESULTS}

We recorded 3,804 individuals belonging to 298 species and to an additional eight taxa that could not

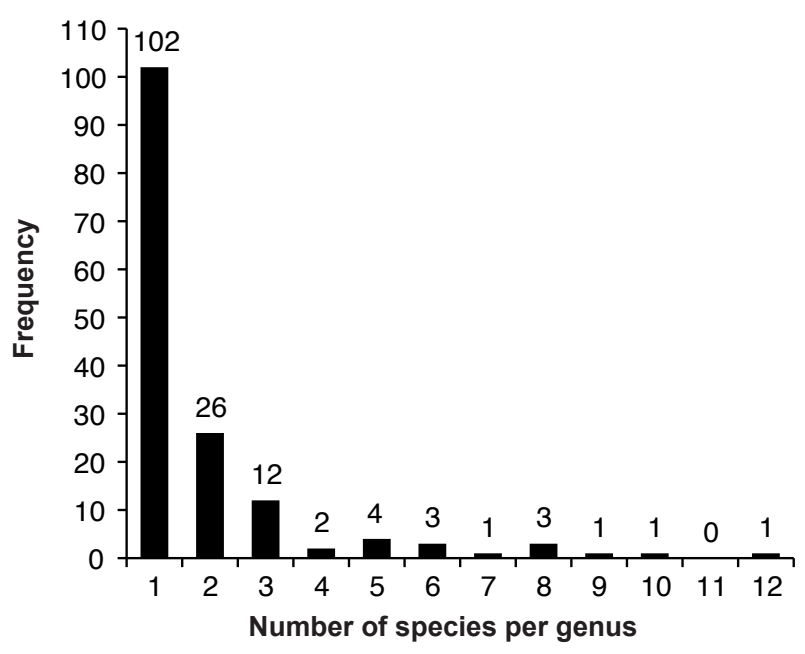

Figure 1. Frequency of the number of species recorded per genus during the present surveys. The number of observations in each category is shown on top of the bars. Most genera were represented by single species.

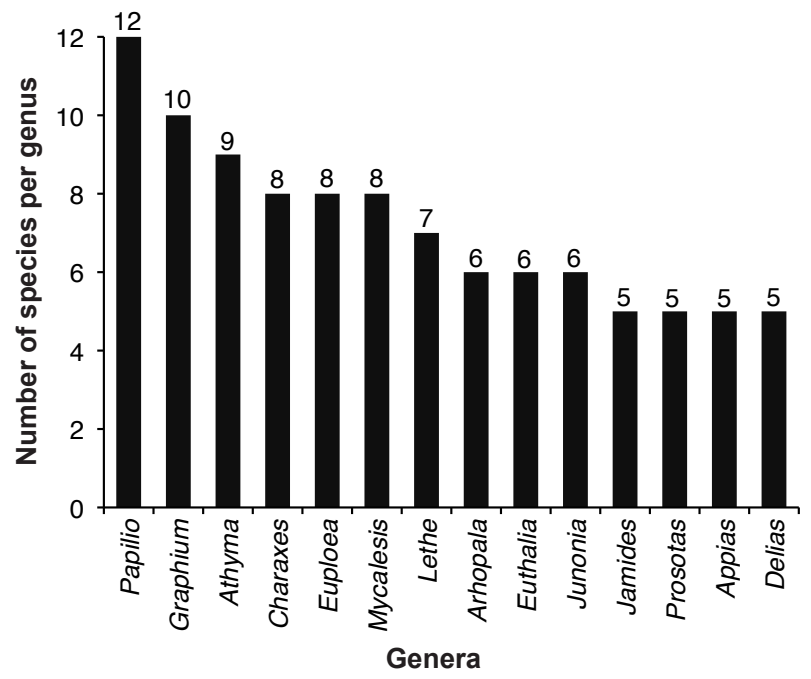

Figure 2. Most species-rich genera of the Garo Hills. All genera with five or more species recorded in the Garo Hills are shown.

be identified to species level (the latter appear in the annotated checklist below and in Table 5 as Mycalesis sp., Byasa sp., Neptis nata/soma group, etc.). These represented 156 genera in 22 subfamilies and six families. A detailed taxonomic breakdown of the Garo Hills butterflies is presented in Tables 1 and 2 .

The distribution of butterfly species across genera was highly skewed. A large proportion of genera (102 out of 156) recorded in our surveys were represented by single species, 26 genera by two species, 12 genera by three species, and two genera by four species, the rest (14 out of 156) by four species or more (Fig. 1). 


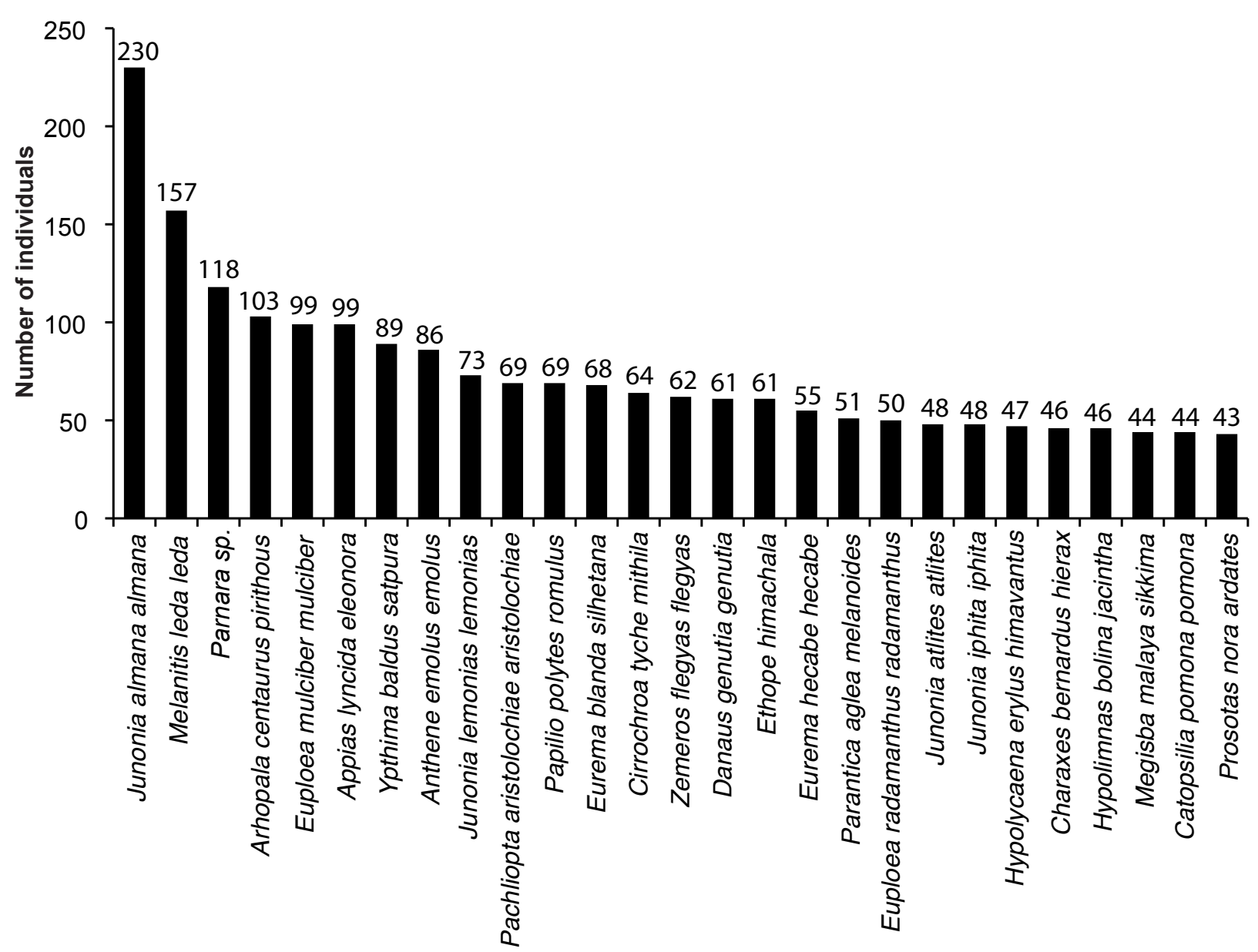

Figure 3. Most common butterfly species of the Garo Hills. These were a mixture of disturbed forest or open habitat species such as Junonia almana almana, Parnara sp., and Catopsilia pomona pomona, as well as evergreen forest species such as Cirrochroa tyche and Ethope himachala.

Table 3. Locality- and season-wise species richness of the Garo Hills butterflies. The numbers of species seen only in one season are given in parentheses for each season and locality.

\begin{tabular}{|l|c|c|c|c|c|}
\hline & $\begin{array}{c}\text { Number of species } \\
\text { seen }\end{array}$ & $\begin{array}{c}\text { Balpakram National } \\
\text { Park }\end{array}$ & $\begin{array}{c}\text { Baghmara Reserve } \\
\text { Forest }\end{array}$ & $\begin{array}{c}\text { Siju Wildlife } \\
\text { Sanctuary }\end{array}$ & $\begin{array}{c}\text { Nokrek National } \\
\text { Park }\end{array}$ \\
\hline Summer & $210(70)$ & $151(53)$ & $143(96)$ & $71(60)$ & - \\
\hline Post-monsoon & $236(96)$ & $193(95)$ & $93(46)$ & $34(23)$ & 57 \\
\hline Both seasons & 140 & 98 & 47 & 11 & - \\
\hline Total & $\begin{array}{c}306(298 \text { identified to } \\
\text { sub/species level) }\end{array}$ & 246 & 189 & 94 & 57 \\
\hline
\end{tabular}

The most prevalent genera - those with five species or more-are listed in Fig. 2. Similarly, the abundance of individual species was highly skewed. The commonest 27 species, i.e., those represented in our sample by 40 individuals or more, or on average at least one individual per count, are shown in Fig. 3. The seasonand locality-wise species richness is given in Table 3, although the differences here may merely reflect our uneven sampling effort.
Although we report a substantial number of butterfly species, the actual butterfly species richness in the Garo Hills appears to be much higher. New species accumulated fairly steeply even after reaching 298 species in 49 days of field work (Fig. 4). The steepness of species accumulation based on sampling from only two seasons, and the known butterfly fauna of the nearby Khasi Hills, suggest that another 300350 species may still be remaining to be discovered 
Table 4. Butterflies legally protected by the Indian Wildlife (Protection) Act, 1972 (WPA), reported here from the Garo Hills in this study. Also see Table 1.

\begin{tabular}{|c|c|c|}
\hline WPA Schedule I & WPA Schedule II & WPA Schedule IV \\
\hline $\begin{array}{l}\text { Lycaenidae: } \\
\text { Allotinus drumila drumila } \\
\text { Chliaria othona othona } \\
\text { Nymphalidae: } \\
\text { Charaxes moori sandakanus } \\
\text { Elymnias peali } \\
\text { Euthalia telchinia } \\
\text { Hypolimnas misippus } \\
\text { Prothoe franck regalis } \\
\text { Symbrenthia silana }\end{array}$ & $\begin{array}{l}\text { Hesperiidae: } \\
\text { Bibasis sena sena } \\
\text { Lycaenidae: } \\
\text { Ancema blanka minturna } \\
\text { Anthene lycaenina lycambes } \\
\text { Arhopala fulla ignara } \\
\text { Bindahara phocides phocides } \\
\text { Euchrysops cnejus cnejus } \\
\text { Flos apidanus ahamus } \\
\text { Horaga onyx onyx } \\
\text { Prosotas aluta coelestis }\end{array}$ & $\begin{array}{l}\text { Hesperiidae: } \\
\text { Baoris farri } \\
\text { Hyarotis adrastus praba } \\
\text { Pelopidas assamensis } \\
\text { Polytremis lubricans lubricans } \\
\text { Lycaenidae: } \\
\text { Tarucus ananda } \\
\text { Nymphalidae: } \\
\text { Euploea mulciber mulciber } \\
\text { Euthalia lubentina lubentina }\end{array}$ \\
\hline $\begin{array}{l}\text { Total no. of protected species under } \\
\text { WPA Schedule I: } 8\end{array}$ & $\begin{array}{l}\text { Rapala varuna orseis } \\
\text { Spindasis lohita himalayanus } \\
\text { Yasoda tripunctata tripunctata } \\
\text { Nymphalidae: } \\
\text { Amathuxidia amythaon amythaon } \\
\text { Athyma asura asura } \\
\text { Athyma pravara acutipennis } \\
\text { Athyma ranga ranga } \\
\text { Bassarona teuta teuta } \\
\text { Bhagadatta austenia austenia } \\
\text { Charaxes marmax marmax } \\
\text { Charaxes solon sulphureus } \\
\text { Cyrestis cocles cocles } \\
\text { Euploea midamus rogenhoferi } \\
\text { Euthalia aconthea garuda } \\
\text { Lexias cyanipardus cyanipardus } \\
\text { Lexias dirtea khasiana } \\
\text { Melanitis zitenius zitenius } \\
\text { Mycalesis anaxias aemate } \\
\text { Mycalesis malsarida } \\
\text { Parthenos sylvia gambrisius } \\
\text { Tanaecia lepidea lepidea } \\
\text { Papilionidae: } \\
\text { Graphium aristeus anticrates } \\
\text { Papilio paradoxa telearchus } \\
\text { Pieridae: } \\
\text { Appias albina darada } \\
\text { Total no. of protected species under WPA } \\
\text { Schedule II: } 33\end{array}$ & $\begin{array}{l}\text { Total no. of protected species under WPA } \\
\text { Schedule IV: } 7\end{array}$ \\
\hline
\end{tabular}

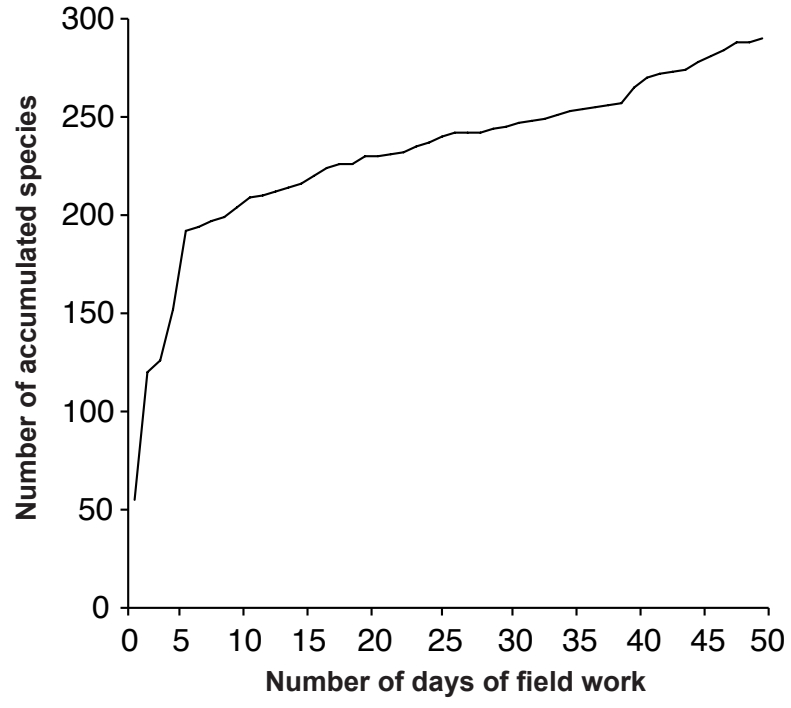

Figure 4. Species accumulation curve for butterflies of the Garo Hills. in the Garo Hills. Nonetheless, the butterfly fauna of the Garo Hills reported here has already proved to be an extremely valuable conservation asset: 48 of the already recorded 298 butterfly species, or approximately $16 \%$, are legally protected in India under the Wildlife (Protection) Act, 1972 (Anonymous 1997) (Table 4). Of these, eight are protected under Schedule I and 33 are protected under Schedule II of the Wildlife (Protection) Act, 1972. These species are very important as they receive the highest level of legal protection at the national level. The taxonomic and Schedule-wise breakdown of legally protected butterfly species found in the Garo Hills is given in Tables 1 and 4. Thus, the Garo Hills not only possess high butterfly species richness, but they also support a large number of rare and legally protected species. The Garo Hills should therefore be considered a toppriority, high-value conservation area for long-term 
Table 5. Locality- and season-wise occurrence and abundance of butterflies of the Garo Hills.

BNP - Balpakram National Park; BRF - Baghmara Reserve Forest; SIJU - Siju Wildlife Sanctuary; NNP - Nokrek National Park;

SUM - summer; WIN - winter.

\begin{tabular}{|c|c|c|c|c|c|c|c|c|c|c|}
\hline No & Scientific name & English name & $\begin{array}{l}\text { BNP } \\
\text { SUM }\end{array}$ & $\begin{array}{l}\text { BNP } \\
\text { WIN }\end{array}$ & $\begin{array}{l}\text { BRF } \\
\text { SUM }\end{array}$ & $\begin{array}{l}\text { BRF } \\
\text { WIN }\end{array}$ & $\begin{array}{l}\text { SIJU } \\
\text { SUM }\end{array}$ & $\begin{array}{l}\text { SIJU } \\
\text { WIN }\end{array}$ & $\begin{array}{l}\text { NNP } \\
\text { WIN }\end{array}$ & Total \\
\hline \multicolumn{11}{|c|}{ Family Hesperiidae, Subfamily Coeliadinae: } \\
\hline 1 & Badamia exclamationis & Brown Awl & 4 & 1 & 1 & 0 & 0 & 0 & 0 & 6 \\
\hline 2 & Bibasis sena sena & Indian Orangetail Awl & 0 & 0 & 1 & 0 & 0 & 0 & 0 & 1 \\
\hline 3 & Burara oedipodea belesis & Himalayan Branded Orange Awlet & 1 & 0 & 0 & 0 & 1 & 0 & 0 & 2 \\
\hline 4 & Choaspes benjaminii japonica & Narrow Indian Awlking & 0 & 1 & 1 & 0 & 0 & 0 & 0 & 2 \\
\hline 5 & Hasora badra badra & Oriental Common Awl & 0 & 0 & 0 & 0 & 1 & 0 & 0 & 1 \\
\hline 6 & Hasora taminatus bhavara & Himalayan White-banded Awl & 0 & 1 & 0 & 0 & 0 & 0 & 0 & 1 \\
\hline \multicolumn{11}{|c|}{ Family Hesperiidae, Subfamily Hesperiinae: } \\
\hline 7 & Aeromachus jhora creta & Khasi Jhora Scrub Hopper & 0 & 0 & 2 & 0 & 0 & 0 & 0 & 2 \\
\hline 8 & Ancistroides nigrita diocles & Bengal Chocolate Demon & 0 & 4 & 1 & 0 & 0 & 1 & 0 & 6 \\
\hline 9 & Baoris chapmani & Small Paint-brush Swift & 0 & 0 & 0 & 1 & 0 & 0 & 0 & 1 \\
\hline 10 & Baoris farri & Complete Paint-brush Swift & 0 & 8 & 0 & 2 & 0 & 0 & 0 & 10 \\
\hline 11 & Baoris unicolor & Black Paint-brush Swift & 0 & 0 & 1 & 0 & 0 & 0 & 0 & 1 \\
\hline 12 & Caltoris sp. & Swift sp. & 0 & 1 & 0 & 0 & 0 & 0 & 0 & 1 \\
\hline 13 & Cupitha purreea & Wax Dart & 0 & 1 & 1 & 0 & 0 & 0 & 0 & 2 \\
\hline 14 & Halpe spp. & Ace spp. & 0 & 2 & 0 & 1 & 1 & 0 & 0 & 4 \\
\hline 15 & Halpe zema zema & Sikkim Zema Banded Ace & 0 & 3 & 0 & 2 & 2 & 0 & 0 & 7 \\
\hline 16 & Hyarotis adrastus praba & Bengal Tree Flitter & 0 & 0 & 0 & 1 & 0 & 0 & 0 & 1 \\
\hline 17 & lambrix salsala salsala & Eastern Chestnut Bob & 2 & 11 & 1 & 0 & 0 & 5 & 0 & 19 \\
\hline 18 & Iton semamora semamora & Bengal Common Wight & 0 & 1 & 0 & 0 & 0 & 0 & 0 & 1 \\
\hline 19 & Koruthaialos butleri & Dark Velvet Bob & 0 & 1 & 0 & 0 & 0 & 0 & 0 & 1 \\
\hline 20 & Matapa aria & Common Branded Redeye & 0 & 4 & 0 & 1 & 0 & 0 & 0 & 5 \\
\hline 21 & Matapa cresta & Fringed Branded Redeye & 0 & 3 & 0 & 0 & 0 & 1 & 0 & 4 \\
\hline 22 & Matapa sasivarna & Black-veined Branded Redeye & 0 & 1 & 0 & 0 & 0 & 0 & 0 & 1 \\
\hline 23 & Notocrypta curvifascia curvifascia & Chinese Restricted Demon & 0 & 3 & 0 & 0 & 0 & 1 & 0 & 4 \\
\hline 24 & Notocrypta feisthamelii alysos & Himalayan Spotted Demon & 0 & 1 & 1 & 0 & 0 & 0 & 0 & 2 \\
\hline 25 & Notocrypta paralysos asawa & $\begin{array}{l}\text { Indo-Chinese Common Banded } \\
\text { Demon }\end{array}$ & 0 & 0 & 0 & 2 & 0 & 0 & 0 & 2 \\
\hline 26 & Oriens gola pseudolus & Oriental Common Dartlet & 0 & 1 & 0 & 0 & 0 & 0 & 0 & 1 \\
\hline 27 & Oriens goloides & Smaller Dartlet & 0 & 3 & 0 & 0 & 0 & 0 & 1 & 4 \\
\hline 28 & Parnara sp. & Swift sp. & 15 & 2 & 101 & 0 & 0 & 0 & 0 & 118 \\
\hline 29 & Pelopidas agna agna & Bengal Obscure Branded Swift & 0 & 1 & 0 & 0 & 0 & 0 & 0 & 1 \\
\hline 30 & Pelopidas assamensis & Great Swift & 2 & 2 & 0 & 0 & 0 & 0 & 0 & 4 \\
\hline 31 & Pirdana major & Himalayan Green-striped Palmer & 0 & 1 & 0 & 0 & 0 & 0 & 0 & 1 \\
\hline
\end{tabular}




\begin{tabular}{|c|c|c|c|c|c|c|c|c|c|c|}
\hline No & Scientific name & English name & $\begin{array}{l}\text { BNP } \\
\text { SUM }\end{array}$ & $\begin{array}{l}\text { BNP } \\
\text { WIN }\end{array}$ & $\begin{array}{l}\text { BRF } \\
\text { SUM }\end{array}$ & $\begin{array}{l}\text { BRF } \\
\text { WIN }\end{array}$ & $\begin{array}{l}\text { SIJU } \\
\text { SUM }\end{array}$ & $\begin{array}{l}\text { SIJU } \\
\text { WIN }\end{array}$ & $\begin{array}{l}\text { NNP } \\
\text { WIN }\end{array}$ & Total \\
\hline 32 & Pithauria stramineipennis stramineipennis & Assam Light Straw Ace & 0 & 0 & 1 & 0 & 2 & 0 & 0 & 3 \\
\hline 33 & Polytremis lubricans lubricans & Oriental Contiguous Swift & 0 & 0 & 1 & 0 & 0 & 0 & 0 & 1 \\
\hline 34 & Potanthus spp. & Dart spp & 0 & 1 & 0 & 0 & 0 & 1 & 0 & 2 \\
\hline 35 & Psolos fuligo subfasciatus & Indian Dusky Partwing & 0 & 1 & 0 & 0 & 0 & 0 & 1 & 2 \\
\hline 36 & Scobura isota & Khasi Forest Bob & 0 & 2 & 0 & 1 & 0 & 0 & 0 & 3 \\
\hline 37 & Sebastonyma dolopia & Tufted Ace & 0 & 0 & 0 & 0 & 3 & 0 & 0 & 3 \\
\hline 38 & Spialia galba & Indian Grizzled Skipper & 1 & 1 & 0 & 0 & 0 & 0 & 0 & 2 \\
\hline 39 & Suada swerga swerga & Indian Grass Bob & 0 & 5 & 0 & 0 & 0 & 0 & 0 & 5 \\
\hline 40 & Telicota bambusae bambusae & Oriental Dark Palm-Dart & 0 & 1 & 0 & 3 & 0 & 0 & 1 & 5 \\
\hline 41 & Zographetus ogygia ogygia & Sumatran Purple-spotted Flitter & 0 & 0 & 0 & 1 & 0 & 0 & 0 & 1 \\
\hline 42 & Zographetus satwa & Purple and Gold Flitter & 0 & 0 & 0 & 1 & 0 & 0 & 0 & 1 \\
\hline
\end{tabular}

Family Hesperiidae, Subfamily Pyrginae:

\begin{tabular}{|c|c|c|c|c|c|c|c|c|c|c|}
\hline 43 & Celaenorrhinus asmara consertus & Khasi White-banded Flat & 0 & 0 & 1 & 0 & 0 & 0 & 0 & 1 \\
\hline 44 & Gerosis bhagava bhagava & Bengal Yellow-breasted Flat & 0 & 0 & 0 & 2 & 0 & 0 & 0 & 2 \\
\hline 45 & Gerosis phisara phisara & Khasi Dusky Yellow-breasted Flat & 1 & 0 & 2 & 1 & 0 & 0 & 0 & 4 \\
\hline 46 & Gerosis sinica narada & Sikkim White Yellow-breasted Flat & 0 & 1 & 0 & 0 & 0 & 0 & 0 & 1 \\
\hline 47 & Odontoptilum angulata angulata & Oriental Chestnut Angle & 10 & 0 & 11 & 0 & 2 & 0 & 0 & 23 \\
\hline 48 & Pseudocoladenia dan fabia & Himalayan Fulvous Pied Flat & 1 & 5 & 1 & 0 & 0 & 0 & 0 & 7 \\
\hline 49 & Sarangesa dasahara dasahara & Bengal Common Small Flat & 4 & 3 & 0 & 1 & 0 & 2 & 1 & 11 \\
\hline 50 & Tagiades gana athos & Bengal Suffused Snow Flat & 0 & 6 & 1 & 2 & 0 & 0 & 0 & 9 \\
\hline 51 & Tagiades japetus ravi & Himalayan Common Snow Flat & 1 & 0 & 2 & 3 & 2 & 0 & 0 & 8 \\
\hline 52 & Tagiades litigiosa litigiosa & Sylhet Water Snow Flat & 0 & 1 & 0 & 0 & 0 & 0 & 0 & 1 \\
\hline
\end{tabular}

Family Lycaenidae, Subfamily Curetinae:

\begin{tabular}{|l|l|l|c|c|c|c|c|c|c|}
\hline 53 & Curetis bulis bulis & Himalayan Bright Sunbeam & 1 & 0 & 0 & 0 & 0 & 0 & 0 \\
\hline 54 & Curetis dentata dentata & Toothed Sunbeam & 6 & 0 & 0 & 0 & 0 & 0 & 0 \\
\hline
\end{tabular}

Family Lycaenidae, Subfamily Lycaeninae:

\begin{tabular}{|c|c|c|c|c|c|c|c|c|c|c|}
\hline 55 & Heliophorus epicles latilimbata & Sikkim Purple Sapphire & 2 & 2 & 1 & 0 & 1 & 0 & 7 & 13 \\
\hline 56 & Heliophorus indicus & Indian Sapphire & 0 & 0 & 0 & 0 & 0 & 0 & 1 & 1 \\
\hline
\end{tabular}

Family Lycaenidae, Subfamily Miletinae:

\begin{tabular}{|c|c|c|c|c|c|c|c|c|c|c|}
\hline 57 & Allotinus drumila drumila & Himalayan Crenulate Mottle & 1 & 0 & 0 & 0 & 0 & 0 & 0 & 1 \\
\hline 58 & Miletus chinensis assamensis & Assam Common Mottle & 0 & 0 & 2 & 0 & 0 & 0 & 0 & 2 \\
\hline 59 & Spalgis epeus epeus & Oriental Apefly & 0 & 0 & 2 & 0 & 0 & 0 & 0 & 2 \\
\hline
\end{tabular}

Family Lycaenidae, Subfamily Polyommatinae:

\begin{tabular}{|c|c|c|c|c|c|c|c|c|c|c|}
\hline 60 & Acytolepis puspa gisca & Himalayan Common Hedge Blue & 1 & 0 & 1 & 0 & 1 & 0 & 0 & 3 \\
\hline 61 & Anthene emolus emolus & Bengal Common Ciliate Blue & 5 & 32 & 36 & 10 & 3 & 0 & 0 & 86 \\
\hline
\end{tabular}




\begin{tabular}{|c|c|c|c|c|c|c|c|c|c|c|}
\hline No & Scientific name & English name & $\begin{array}{l}\text { BNP } \\
\text { SUM }\end{array}$ & $\begin{array}{l}\text { BNP } \\
\text { WIN }\end{array}$ & $\begin{array}{l}\text { BRF } \\
\text { SUM }\end{array}$ & $\begin{array}{l}\text { BRF } \\
\text { WIN }\end{array}$ & $\begin{array}{l}\text { SIJU } \\
\text { SUM }\end{array}$ & $\begin{array}{l}\text { SIJU } \\
\text { WIN }\end{array}$ & $\begin{array}{l}\text { NNP } \\
\text { WIN }\end{array}$ & Total \\
\hline 62 & Anthene lycaenina lycambes & Shan Pointed Ciliate Blue & 0 & 3 & 0 & 1 & 0 & 0 & 0 & 4 \\
\hline 63 & Caleta elna noliteia & Indo-Chinese Elbowed Pierrot & 4 & 4 & 1 & 2 & 2 & 0 & 0 & 13 \\
\hline 64 & Castalius rosimon rosimon & Continental Common Pierrot & 3 & 3 & 2 & 0 & 1 & 0 & 0 & 9 \\
\hline 65 & Catochrysops panormus exiguus & Malay Silver Forget-me-not & 1 & 1 & 0 & 0 & 0 & 0 & 0 & 2 \\
\hline 66 & Catochrysops strabo strabo & Oriental Forget-me-not & 0 & 1 & 0 & 0 & 1 & 0 & 0 & 2 \\
\hline 67 & Celastrina lavendularis limbata & Eastern Plain Hedge Blue & 3 & 5 & 0 & 1 & 1 & 1 & 0 & 11 \\
\hline 68 & Chilades lajus lajus & Indian Lime Blue & 1 & 0 & 2 & 0 & 0 & 0 & 0 & 3 \\
\hline 69 & Discolampa ethion ethion & Oriental Banded Blue Pierrot & 0 & 1 & 0 & 0 & 0 & 0 & 0 & 1 \\
\hline 70 & Euchrysops cnejus cnejus & Oriental Gram Blue & 0 & 1 & 0 & 0 & 0 & 0 & 1 & 2 \\
\hline 71 & Jamides alecto eurysaces & Himalayan Metallic Cerulean & 6 & 0 & 0 & 0 & 1 & 0 & 0 & 7 \\
\hline 72 & Jamides bochus bochus & Indian Dark Cerulean & 1 & 0 & 0 & 4 & 0 & 0 & 0 & 5 \\
\hline 73 & Jamides celeno celeno & Oriental Common Cerulean & 2 & 7 & 12 & 0 & 2 & 1 & 0 & 24 \\
\hline 74 & Jamides elpis pseudelpis & False Glistening Cerulean & 0 & 0 & 0 & 0 & 1 & 0 & 0 & 1 \\
\hline 75 & Jamides pura pura & Continental White Cerulean & 0 & 4 & 0 & 0 & 0 & 0 & 0 & 4 \\
\hline 76 & Lampides boeticus & Pea Blue & 0 & 0 & 0 & 0 & 0 & 0 & 1 & 1 \\
\hline 77 & Lestranicus transpectus & White-banded Hedge Blue & 0 & 0 & 1 & 0 & 0 & 0 & 0 & 1 \\
\hline 78 & Megisba malaya sikkima & Variable Malayan & 21 & 1 & 16 & 1 & 5 & 0 & 0 & 44 \\
\hline 79 & Nacaduba beroe gythion & Assam Opaque Six-Lineblue & 1 & 3 & 0 & 5 & 0 & 0 & 0 & 9 \\
\hline 80 & Nacaduba hermus nabo & Assam Pale Four-Lineblue & 0 & 2 & 0 & 1 & 0 & 0 & 0 & 3 \\
\hline 81 & Nacaduba kurava euplea & Sikkim Transparent Six-Lineblue & 0 & 0 & 0 & 3 & 0 & 0 & 0 & 3 \\
\hline 82 & Neopithecops zalmora zalmora & Myanmar Common Quaker & 4 & 0 & 2 & 2 & 0 & 0 & 0 & 8 \\
\hline 83 & Prosotas aluta coelestis & Assam Banded Lineblue & 1 & 1 & 3 & 0 & 0 & 0 & 0 & 5 \\
\hline 84 & Prosotas bhutea & Bhutya Lineblue & 2 & 0 & 0 & 0 & 0 & 0 & 0 & 2 \\
\hline 85 & Prosotas dubiosa indica & Indian Tailless Lineblue & 10 & 1 & 6 & 10 & 1 & 0 & 0 & 28 \\
\hline 86 & Prosotas lutea sivoka & Teesta Brown Lineblue & 1 & 10 & 0 & 0 & 0 & 0 & 0 & 11 \\
\hline 87 & Prosotas nora ardates & Indian Common Lineblue & 1 & 26 & 3 & 12 & 0 & 0 & 1 & 43 \\
\hline 88 & Pseudozizeeria maha maha & Himalayan Pale Grass Blue & 0 & 0 & 1 & 0 & 4 & 0 & 0 & 5 \\
\hline 89 & Talicada nyseus khasiana & Khasi Red Pierrot & 0 & 2 & 0 & 0 & 0 & 0 & 0 & 2 \\
\hline 90 & Tarucus ananda & Dark Pierrot & 1 & 0 & 1 & 0 & 0 & 0 & 0 & 2 \\
\hline 91 & Tarucus venosus & Veined Pierrot & 0 & 1 & 0 & 0 & 0 & 0 & 0 & 1 \\
\hline 92 & Udara dilecta dilecta & Himalayan Pale Hedge Blue & 0 & 0 & 0 & 1 & 0 & 0 & 0 & 1 \\
\hline 93 & Zizeeria karsandra & Dark Grass Blue & 0 & 0 & 1 & 0 & 0 & 0 & 0 & 1 \\
\hline 94 & Zizina otis otis & Oriental Lesser Grass Blue & 0 & 2 & 0 & 0 & 0 & 0 & 0 & 2 \\
\hline 95 & Zizula hylax hylax & Indian Tiny Grass Blue & 0 & 0 & 1 & 0 & 0 & 0 & 0 & 1 \\
\hline
\end{tabular}




\begin{tabular}{|c|c|c|c|c|c|c|c|c|c|c|}
\hline No & Scientific name & English name & $\begin{array}{l}\text { BNP } \\
\text { SUM }\end{array}$ & $\begin{array}{l}\text { BNP } \\
\text { WIN }\end{array}$ & $\begin{array}{l}\text { BRF } \\
\text { SUM }\end{array}$ & $\begin{array}{l}\text { BRF } \\
\text { WIN }\end{array}$ & $\begin{array}{l}\text { SIJU } \\
\text { SUM }\end{array}$ & $\begin{array}{l}\text { SIJU } \\
\text { WIN }\end{array}$ & $\begin{array}{l}\text { NNP } \\
\text { WIN }\end{array}$ & Total \\
\hline \multicolumn{11}{|c|}{ Family Lycaenidae, Subfamily Theclinae: } \\
\hline 96 & Amblypodia anita dina & Indian Purple Leaf Blue & 1 & 0 & 0 & 0 & 0 & 0 & 0 & 1 \\
\hline 97 & Ancema blanka minturna & Blue-streaked Silver Royal & 0 & 3 & 0 & 0 & 0 & 0 & 0 & 3 \\
\hline 98 & Ancema ctesia ctesia & Himalayan Bi-spot Royal & 0 & 0 & 0 & 1 & 0 & 0 & 0 & 1 \\
\hline 99 & Arhopala abseus indicus & Indian Aberrant Oakblue & 0 & 0 & 1 & 0 & 0 & 0 & 0 & 1 \\
\hline 100 & Arhopala ammonides elira & Assam Little Cerulean Oakblue & 0 & 0 & 1 & 0 & 0 & 0 & 0 & 1 \\
\hline 101 & Arhopala atrax & Indian Oakblue & 0 & 0 & 1 & 0 & 0 & 0 & 0 & 1 \\
\hline 102 & Arhopala centaurus pirithous & Bengal Centaur Oakblue & 1 & 0 & 100 & 2 & 0 & 0 & 0 & 103 \\
\hline 103 & Arhopala fulla ignara & Thai Spotless Oakblue & 1 & 0 & 14 & 0 & 0 & 0 & 0 & 15 \\
\hline 104 & Arhopala perimuta perimuta & Sylhet Yellowdisc Tailless Oakblue & 3 & 0 & 3 & 0 & 0 & 0 & 0 & 6 \\
\hline 105 & Bindahara phocides phocides & Thai Plane & 0 & 0 & 2 & 0 & 0 & 0 & 0 & 2 \\
\hline 106 & Cheritra freja evansi & Khasi Common Imperial & 1 & 1 & 0 & 1 & 0 & 0 & 0 & 3 \\
\hline 107 & Chliaria othona othona & Oriental Orchid Tit & 1 & 0 & 1 & 1 & 1 & 0 & 0 & 4 \\
\hline 108 & Creon cleobis cleobis & Bengal Broad-tail Royal & 0 & 0 & 1 & 0 & 0 & 0 & 0 & 1 \\
\hline 109 & Dacalana penicilligera & Double-tufted Royal & 0 & 0 & 1 & 0 & 0 & 0 & 0 & 1 \\
\hline 110 & Flos apidanus ahamus & Assam Plain Plushblue & 0 & 0 & 0 & 0 & 0 & 1 & 0 & 1 \\
\hline 111 & Horaga onyx onyx & Variable Common Onyx & 0 & 1 & 1 & 0 & 0 & 0 & 0 & 2 \\
\hline 112 & Hypolycaena erylus himavantus & Sikkim Common Tit & 1 & 25 & 4 & 16 & 0 & 0 & 1 & 47 \\
\hline 113 & Loxura atymnus continentalis & Continental Yamfly & 4 & 3 & 3 & 0 & 0 & 0 & 0 & 10 \\
\hline 114 & Lycaenid sp. & & 0 & 0 & 0 & 1 & 0 & 0 & 0 & 1 \\
\hline 115 & Rapala dieneces dieneces & Malay Scarlet Flash & 0 & 2 & 0 & 0 & 0 & 0 & 0 & 2 \\
\hline 116 & Rapala manea schistacea & Bengal Slate Flash & 1 & 0 & 2 & 0 & 0 & 0 & 0 & 3 \\
\hline 117 & Rapala pheretima petosiris & Indian Copper Flash & 1 & 0 & 3 & 1 & 0 & 0 & 0 & 5 \\
\hline 118 & Rapala varuna orseis & Sumatran Indigo Flash & 0 & 0 & 0 & 1 & 0 & 1 & 0 & 2 \\
\hline 119 & Remelana jangala ravata & Northern Chocolate Royal & 1 & 1 & 0 & 1 & 0 & 0 & 0 & 3 \\
\hline 120 & Spindasis lohita himalayanus & Himalayan Long-banded Silverline & 0 & 3 & 0 & 0 & 0 & 1 & 0 & 4 \\
\hline 121 & Spindasis syama peguanus & Pegu Club Silverline & 2 & 4 & 0 & 0 & 0 & 0 & 0 & 6 \\
\hline 122 & Surendra quercetorum quercetorum & Himalayan Common Acacia Blue & 0 & 2 & 6 & 0 & 0 & 0 & 0 & 8 \\
\hline 123 & Ticherra acte acte & Himalayan Blue Imperial & 1 & 0 & 0 & 0 & 0 & 0 & 0 & 1 \\
\hline 124 & Yasoda tripunctata tripunctata & Sylhet Branded Yamfly & 1 & 0 & 1 & 0 & 0 & 0 & 0 & 2 \\
\hline 125 & Zeltus amasa amasa & Indian Fluffy Tit & 17 & 8 & 4 & 0 & 0 & 0 & 0 & 29 \\
\hline \multicolumn{11}{|c|}{ Family Nymphalidae, Subfamily Apaturinae: } \\
\hline 126 & Apatura ambica ambica & East Himalayan Purple Emperor & 1 & 1 & 0 & 0 & 0 & 0 & 0 & 2 \\
\hline 127 & Euripus nyctelius nyctelius & Sylhet Courtesan & 2 & 5 & 0 & 0 & 0 & 0 & 0 & 7 \\
\hline 128 & Herona marathus marathus & Assam Pasha & 0 & 0 & 0 & 0 & 0 & 0 & 1 & 1 \\
\hline
\end{tabular}




\begin{tabular}{|c|c|c|c|c|c|c|c|c|c|c|}
\hline No & Scientific name & English name & $\begin{array}{l}\text { BNP } \\
\text { SUM }\end{array}$ & $\begin{array}{l}\text { BNP } \\
\text { WIN }\end{array}$ & $\begin{array}{l}\text { BRF } \\
\text { SUM }\end{array}$ & $\begin{array}{l}\text { BRF } \\
\text { WIN }\end{array}$ & $\begin{array}{l}\text { SIJU } \\
\text { SUM }\end{array}$ & $\begin{array}{l}\text { SIJU } \\
\text { WIN }\end{array}$ & $\begin{array}{l}\text { NNP } \\
\text { WIN }\end{array}$ & Total \\
\hline 129 & Rohana parisatis parisatis & Assam Black Prince & 0 & 2 & 0 & 0 & 1 & 0 & 0 & 3 \\
\hline \multicolumn{11}{|c|}{ Family Nymphalidae, Subfamily Biblidinae: } \\
\hline 130 & Ariadne ariadne pallidior & Large Angled Castor & 6 & 2 & 4 & 0 & 3 & 0 & 0 & 15 \\
\hline 131 & Ariadne merione tapestrina & Intricate Common Castor & 0 & 4 & 1 & 0 & 0 & 0 & 0 & 5 \\
\hline \multicolumn{11}{|c|}{ Family Nymphalidae, Subfamily Charaxinae: } \\
\hline 132 & Charaxes arja arja & Bengal Pallid Nawab & 2 & 7 & 0 & 0 & 0 & 0 & 0 & 9 \\
\hline 133 & Charaxes athamas athamas & Oriental Common Nawab & 2 & 32 & 0 & 1 & 0 & 0 & 0 & 35 \\
\hline 134 & Charaxes bernardus hierax & Variable Tawny Rajah & 8 & 5 & 9 & 1 & 23 & 0 & 0 & 46 \\
\hline 135 & Charaxes eudamippus eudamippus & Himalayan Great Nawab & 1 & 0 & 0 & 0 & 0 & 0 & 0 & 1 \\
\hline 136 & Charaxes kahruba & Variegated Rajah & 1 & 2 & 1 & 0 & 0 & 0 & 0 & 4 \\
\hline 137 & Charaxes marmax marmax & Sylhet Yellow Rajah & 0 & 3 & 0 & 0 & 0 & 0 & 0 & 3 \\
\hline 138 & Charaxes moori sandakanus & Margined Malayan Nawab & 1 & 0 & 0 & 0 & 0 & 0 & 0 & 2 \\
\hline 139 & Charaxes solon sulphureus & Sulphur Black Rajah & 0 & 0 & 0 & 0 & 2 & 0 & 0 & 2 \\
\hline 140 & Prothoe franck regalis & Regal Blue Begum & 0 & 2 & 0 & 0 & 0 & 0 & 0 & 2 \\
\hline
\end{tabular}

\section{Family Nymphalidae, Subfamily Cyrestinae:}

\begin{tabular}{|c|c|c|c|c|c|c|c|c|c|c|}
\hline 141 & Chersonesia risa risa & Oriental Common Maplet & 5 & 2 & 0 & 1 & 1 & 1 & 0 & 10 \\
\hline 142 & Cyrestis cocles cocles & Thai Marbled Map Butterfly & 12 & 7 & 3 & 0 & 4 & 0 & 0 & 26 \\
\hline 143 & Cyrestis thyodamas thyodamas & Oriental Map Butterfly & 6 & 2 & 0 & 0 & 0 & 0 & 0 & 8 \\
\hline
\end{tabular}

Family Nymphalidae, Subfamily Danainae:

\begin{tabular}{|c|c|c|c|c|c|c|c|c|c|c|}
\hline 144 & Danaus chrysippus chrysippus & Oriental Plain Tiger & 1 & 0 & 14 & 0 & 2 & 0 & 0 & 17 \\
\hline 145 & Danaus genutia genutia & Oriental Striped Tiger & 30 & 5 & 14 & 0 & 4 & 0 & 8 & 61 \\
\hline 146 & Euploea algea deione & Bengal Long-branded Blue Crow & 4 & 0 & 3 & 0 & 2 & 0 & 0 & 9 \\
\hline 147 & Euploea core core & Indian Common Crow & 15 & 1 & 17 & 0 & 2 & 0 & 1 & 36 \\
\hline 148 & Euploea doubledayi doubledayi & Sylhet Striped Black Crow & 17 & 1 & 7 & 0 & 2 & 0 & 5 & 32 \\
\hline 149 & Euploea klugii klugii & Blue King Crow & 0 & 1 & 0 & 0 & 0 & 0 & 0 & 1 \\
\hline 150 & Euploea midamus rogenhoferi & Assam Blue-spotted Crow & 1 & 0 & 0 & 0 & 0 & 0 & 0 & 1 \\
\hline 151 & Euploea mulciber mulciber & Bengal Striped Blue Crow & 12 & 5 & 22 & 1 & 4 & 5 & 50 & 99 \\
\hline 152 & Euploea radamanthus radamanthus & Oriental Magpie Crow & 23 & 4 & 19 & 0 & 2 & 0 & 2 & 50 \\
\hline 153 & Euploea sylvester hopei & Cachar Double-branded Blue Crow & 4 & 0 & 2 & 0 & 0 & 0 & 0 & 6 \\
\hline 154 & Parantica aglea melanoides & Himalayan Glassy Tiger & 2 & 2 & 6 & 1 & 0 & 7 & 33 & 51 \\
\hline 155 & Parantica melaneus plataniston & Himalayan Chocolate Tiger & 1 & 2 & 0 & 0 & 0 & 0 & 6 & 9 \\
\hline 156 & Parantica sita sita & Kashmir Chestnut Tiger & 1 & 0 & 0 & 0 & 0 & 0 & 6 & 7 \\
\hline 157 & Tirumala limniace exoticus & Oriental Blue Tiger & 4 & 0 & 3 & 1 & 1 & 0 & 0 & 9 \\
\hline 158 & Tirumala septentrionis septentrionis & Oriental Dark Blue Tiger & 11 & 2 & 0 & 0 & 0 & 2 & 0 & 15 \\
\hline
\end{tabular}




\begin{tabular}{|c|c|c|c|c|c|c|c|c|c|c|}
\hline No & Scientific name & English name & $\begin{array}{l}\text { BNP } \\
\text { SUM }\end{array}$ & $\begin{array}{l}\text { BNP } \\
\text { WIN }\end{array}$ & $\begin{array}{l}\text { BRF } \\
\text { SUM }\end{array}$ & $\begin{array}{l}\text { BRF } \\
\text { WIN }\end{array}$ & $\begin{array}{l}\text { SIJU } \\
\text { SUM }\end{array}$ & $\begin{array}{l}\text { SIJU } \\
\text { WIN }\end{array}$ & $\begin{array}{l}\text { NNP } \\
\text { WIN }\end{array}$ & Total \\
\hline \multicolumn{11}{|c|}{ Family Nymphalidae, Subfamily Heliconiinae: } \\
\hline 159 & Argynnis hyperbius hyperbius & Chinese Tropical Fritillary & 0 & 1 & 0 & 0 & 0 & 0 & 1 & 2 \\
\hline 160 & Cethosia biblis tisamena & Himalayan Red Lacewing & 0 & 1 & 0 & 0 & 0 & 0 & 30 & 31 \\
\hline 161 & Cethosia cyane cyane & Bengal Leopard Lacewing & 4 & 1 & 4 & 0 & 0 & 0 & 1 & 10 \\
\hline 162 & Cirrochroa aoris aoris & Himalayan Large Yeoman & 3 & 2 & 1 & 0 & 0 & 0 & 0 & 6 \\
\hline 163 & Cirrochroa tyche mithila & Bengal Common Yeoman & 30 & 16 & 2 & 11 & 5 & 0 & 0 & 64 \\
\hline 164 & Phalanta alcippe alcippoides & Himalayan Small Leopard & 1 & 2 & 3 & 0 & 0 & 0 & 0 & 6 \\
\hline 165 & Phalanta phalantha phalantha & Oriental Common Leopard & 6 & 1 & 4 & 3 & 0 & 0 & 2 & 16 \\
\hline 166 & Vagrans egista sinha & Himalayan Vagrant & 0 & 0 & 0 & 1 & 0 & 0 & 0 & 1 \\
\hline 167 & Vindula erota erota & Thai Cruiser & 2 & 2 & 0 & 0 & 0 & 0 & 0 & 4 \\
\hline
\end{tabular}

Family Nymphalidae, Subfamily Limenitidinae:

\begin{tabular}{|c|c|c|c|c|c|c|c|c|c|c|}
\hline 168 & Athyma asura asura & Himalayan Studded Sergeant & 0 & 0 & 3 & 1 & 0 & 0 & 0 & 4 \\
\hline 169 & Athyma cama cama & Himalayan Orange Staff Sergeant & 0 & 1 & 0 & 0 & 0 & 0 & 1 & 2 \\
\hline 170 & Athyma inara inara & Himalayan Color Sergeant & 3 & 11 & 0 & 10 & 0 & 0 & 0 & 24 \\
\hline 171 & Athyma kanwa phorkys & Northern Dot-dash Sergeant & 0 & 0 & 0 & 1 & 0 & 0 & 0 & 1 \\
\hline 172 & Athyma perius perius & Oriental Common Sergeant & 1 & 1 & 0 & 0 & 0 & 0 & 3 & 5 \\
\hline 173 & Athyma pravara acutipennis & Assam Unbroken Sergeant & 0 & 3 & 0 & 0 & 0 & 0 & 0 & 3 \\
\hline 174 & Athyma ranga ranga & Himalayan Blackvein Sergeant & 1 & 2 & 0 & 0 & 0 & 0 & 0 & 3 \\
\hline 175 & Athyma selenophora bahula & Sylhet Staff Sergeant & 0 & 1 & 0 & 1 & 0 & 0 & 0 & 2 \\
\hline 176 & Athyma zeroca zeroca & Khasi Small Staff Sergeant & 0 & 1 & 0 & 0 & 0 & 0 & 0 & 1 \\
\hline 177 & Bassarona teuta teuta & Sylhet Banded Marquis & 0 & 1 & 0 & 0 & 0 & 0 & 0 & 1 \\
\hline 178 & Bhagadatta austenia austenia & Khasi Grey Commodore & 2 & 0 & 0 & 0 & 0 & 0 & 0 & 2 \\
\hline 179 & Euthalia aconthea garuda & Northern Baron & 0 & 1 & 0 & 1 & 0 & 0 & 0 & 2 \\
\hline 180 & Euthalia anosia anosia & Assam Grey Baron & 0 & 1 & 0 & 0 & 0 & 0 & 0 & 1 \\
\hline 181 & Euthalia Iubentina lubentina & Chinese Gaudy Baron & 1 & 0 & 1 & 1 & 0 & 0 & 0 & 3 \\
\hline 182 & Euthalia monina kesava & Assam Powdered Baron & 0 & 1 & 1 & 5 & 0 & 0 & 0 & 7 \\
\hline 183 & Euthalia phemius phemius & Sylhet White-edged Blue Baron & 0 & 2 & 0 & 0 & 0 & 0 & 0 & 2 \\
\hline 184 & Euthalia telchinia & Blue Baron & 0 & 1 & 0 & 0 & 0 & 0 & 0 & 1 \\
\hline 185 & Lebadea martha martha & Thai Knight & 2 & 5 & 9 & 5 & 0 & 3 & 0 & 24 \\
\hline 186 & Lexias cyanipardus cyanipardus & Sylhet Great Archduke & 1 & 4 & 0 & 0 & 0 & 0 & 0 & 5 \\
\hline 187 & Lexias dirtea khasiana & Khasi Dark Archduke & 0 & 1 & 5 & 0 & 1 & 0 & 0 & 7 \\
\hline 188 & Moduza procris procris & Oriental Commander & 0 & 5 & 0 & 1 & 0 & 0 & 0 & 6 \\
\hline 189 & Neptis hylas varmona & Indian Common Sailer & 4 & 16 & 3 & 1 & 0 & 4 & 1 & 29 \\
\hline 190 & Neptis nata/soma group & Sailer spp. & 3 & 2 & 1 & 1 & 2 & 0 & 0 & 9 \\
\hline 191 & Neptis sappho astola & Himalayan Common Glider & 0 & 1 & 0 & 0 & 0 & 0 & 0 & 1 \\
\hline
\end{tabular}




\begin{tabular}{|c|c|c|c|c|c|c|c|c|c|c|}
\hline No & Scientific name & English name & $\begin{array}{l}\text { BNP } \\
\text { SUM }\end{array}$ & $\begin{array}{l}\text { BNP } \\
\text { WIN }\end{array}$ & $\begin{array}{l}\text { BRF } \\
\text { SUM }\end{array}$ & $\begin{array}{l}\text { BRF } \\
\text { WIN }\end{array}$ & $\begin{array}{l}\text { SIJU } \\
\text { SUM }\end{array}$ & $\begin{array}{l}\text { SIJU } \\
\text { WIN }\end{array}$ & $\begin{array}{l}\text { NNP } \\
\text { WIN }\end{array}$ & Total \\
\hline 192 & Pantoporia hordonia hordonia & Oriental Common Lascar & 0 & 14 & 1 & 0 & 0 & 0 & 1 & 16 \\
\hline 193 & Pantoporia paraka paraka & Oriental Perak Lascar & 0 & 1 & 0 & 1 & 0 & 0 & 0 & 2 \\
\hline 194 & Parthenos sylvia gambrisius & Bengal Clipper & 7 & 3 & 9 & 0 & 1 & 0 & 0 & 20 \\
\hline 195 & Phaedyma columella ophiana & Sikkim Short-banded Sailer & 1 & 1 & 0 & 0 & 0 & 0 & 0 & 2 \\
\hline 196 & Sumalia daraxa daraxa & Sylhet Green Commodore & 0 & 0 & 0 & 0 & 0 & 0 & 1 & 1 \\
\hline 197 & Tanaecia jahnu jahnu & Darjeeling Plain Earl & 0 & 2 & 0 & 0 & 0 & 0 & 0 & 2 \\
\hline 198 & Tanaecia julii appiades & Changeable Common Earl & 0 & 0 & 1 & 0 & 0 & 0 & 0 & 1 \\
\hline 199 & Tanaecia lepidea lepidea & Himalayan Grey Count & 6 & 9 & 14 & 2 & 2 & 3 & 0 & 36 \\
\hline
\end{tabular}

Family Nymphalidae, Subfamily Nymphalinae:

\begin{tabular}{|c|c|c|c|c|c|c|c|c|c|c|}
\hline 200 & Doleschallia bisaltide indica & Himalayan Autumn Leaf & 0 & 0 & 1 & 0 & 0 & 0 & 0 & 1 \\
\hline 201 & Hypolimnas bolina jacintha & Oriental Great Eggfly & 26 & 2 & 15 & 0 & 3 & 0 & 0 & 46 \\
\hline 202 & Hypolimnas misippus & Danaid Eggfly & 4 & 0 & 1 & 0 & 0 & 0 & 0 & 5 \\
\hline 203 & Junonia almana almana & Oriental Peacock Pansy & 104 & 13 & 95 & 1 & 14 & 0 & 3 & 230 \\
\hline 204 & Junonia atlites atlites & Oriental Grey Pansy & 8 & 16 & 13 & 11 & 0 & 0 & 0 & 48 \\
\hline 205 & Junonia hierta hierta & Oriental Yellow Pansy & 8 & 1 & 5 & 11 & 0 & 0 & 0 & 25 \\
\hline 206 & Junonia iphita iphita & Oriental Chocolate Pansy & 16 & 4 & 24 & 2 & 2 & 0 & 0 & 48 \\
\hline 207 & Junonia lemonias lemonias & Chinese Lemon Pansy & 52 & 4 & 15 & 1 & 0 & 0 & 1 & 73 \\
\hline 208 & Junonia orithya ocyale & Dark Blue Pansy & 0 & 1 & 0 & 0 & 0 & 0 & 0 & 1 \\
\hline 209 & Kallima inachus inachus & Himalayan Orange Oakleaf & 7 & 2 & 1 & 0 & 0 & 0 & 0 & 10 \\
\hline 210 & Kaniska canace canace & Chinese Blue Admiral & 0 & 3 & 0 & 0 & 0 & 0 & 0 & 3 \\
\hline 211 & Symbrenthia hypselis cotanda & Himalayan Spotted Jester & 0 & 0 & 0 & 0 & 0 & 0 & 1 & 1 \\
\hline 212 & Symbrenthia lilaea khasiana & Khasi Common Jester & 1 & 8 & 0 & 0 & 1 & 0 & 0 & 10 \\
\hline 213 & Symbrenthia silana & Scarce Jester & 0 & 0 & 0 & 0 & 0 & 0 & 1 & 1 \\
\hline 214 & Vanessa cardui cardui & Painted Lady & 0 & 1 & 0 & 0 & 0 & 0 & 0 & 1 \\
\hline 215 & Vanessa indica indica & Himalayan Red Admiral & 3 & 0 & 0 & 0 & 0 & 0 & 0 & 3 \\
\hline
\end{tabular}

Family Nymphalidae, Subfamily Pseudergolinae:

\begin{tabular}{|c|c|c|c|c|c|c|c|c|c|c|}
\hline 216 & Dichorragia nesimachus nesimachus & Himalayan Constable & 0 & 0 & 1 & 0 & 0 & 0 & 0 & 1 \\
\hline 217 & Stibochiona nicea nicea & Himalayan Popinjay & 0 & 10 & 2 & 1 & 0 & 0 & 1 & 14 \\
\hline
\end{tabular}

Family Nymphalidae, Subfamily Satyrinae:

\begin{tabular}{|c|c|c|c|c|c|c|c|c|c|c|}
\hline 218 & Amathuxidia amythaon amythaon & Sylhet Koh-i-Noor & 0 & 0 & 3 & 0 & 0 & 1 & 0 & 4 \\
\hline 219 & Discophora sondaica zal & Indian Common Duffer & 2 & 2 & 0 & 1 & 0 & 0 & 0 & 5 \\
\hline 220 & Elymnias hypermnestra undularis & Wavy Common Palmfly & 0 & 2 & 13 & 3 & 1 & 1 & 0 & 20 \\
\hline 221 & Elymnias malelas malelas & Bengal Spotted Palmfly & 0 & 0 & 1 & 0 & 0 & 0 & 0 & 1 \\
\hline 222 & Elymnias nesaea timandra & Sylhet Tiger Palmfly & 0 & 1 & 1 & 0 & 0 & 1 & 0 & 3 \\
\hline 223 & Elymnias peali & Brahmaputra Palmfly & 0 & 1 & 2 & 0 & 0 & 0 & 0 & 3 \\
\hline
\end{tabular}




\begin{tabular}{|c|c|c|c|c|c|c|c|c|c|c|}
\hline No & Scientific name & English name & $\begin{array}{l}\text { BNP } \\
\text { SUM }\end{array}$ & $\begin{array}{l}\text { BNP } \\
\text { WIN }\end{array}$ & $\begin{array}{l}\text { BRF } \\
\text { SUM }\end{array}$ & $\begin{array}{l}\text { BRF } \\
\text { WIN }\end{array}$ & $\begin{array}{l}\text { SIJU } \\
\text { SUM }\end{array}$ & $\begin{array}{l}\text { SIJU } \\
\text { WIN }\end{array}$ & $\begin{array}{l}\text { NNP } \\
\text { WIN }\end{array}$ & Total \\
\hline 224 & Ethope himachala & Dusky Diadem & 7 & 22 & 9 & 20 & 3 & 0 & 0 & 61 \\
\hline 225 & Faunis canens arcesilas & Thai Common Faun & 2 & 2 & 0 & 0 & 0 & 1 & 0 & 5 \\
\hline 226 & Faunis eumeus assama & Assam Large Faun & 2 & 0 & 0 & 0 & 0 & 0 & 0 & 2 \\
\hline 227 & Lethe chandica flanona & Assam Angled Red Forester & 0 & 1 & 0 & 0 & 0 & 0 & 1 & 2 \\
\hline 228 & Lethe confusa gambara & Assam Banded Treebrown & 0 & 5 & 0 & 0 & 0 & 1 & 0 & 6 \\
\hline 229 & Lethe europa niladana & Himalayan Bamboo Treebrown & 0 & 1 & 2 & 0 & 1 & 0 & 0 & 4 \\
\hline 230 & Lethe mekara zuchara & Assam Common Red Forester & 2 & 2 & 4 & 2 & 0 & 0 & 0 & 10 \\
\hline 231 & Lethe rohria rohria & Himalayan Common Treebrown & 0 & 0 & 0 & 0 & 0 & 0 & 1 & 1 \\
\hline 232 & Lethe verma sintica & $\begin{array}{l}\text { East Himalayan Straight-banded } \\
\text { Treebrown }\end{array}$ & 2 & 0 & 0 & 0 & 0 & 0 & 1 & 3 \\
\hline 233 & Lethe vindhya vindhya & Assam Black Forester & 1 & 0 & 0 & 1 & 0 & 0 & 0 & 2 \\
\hline 234 & Melanitis leda leda & Oriental Common Evening Brown & 12 & 44 & 32 & 14 & 2 & 50 & 3 & 157 \\
\hline 235 & Melanitis phedima bela & Bengal Dark Evening Brown & 0 & 3 & 0 & 2 & 0 & 2 & 2 & 9 \\
\hline 236 & Melanitis zitenius zitenius & Himalayan Great Evening Brown & 0 & 1 & 0 & 0 & 1 & 1 & 0 & 3 \\
\hline 237 & Mycalesis anaxias aemate & Indo-Chinese White-bar Bushbrown & 2 & 2 & 0 & 1 & 1 & 1 & 0 & 7 \\
\hline 238 & Mycalesis francisca sanatana & Himalayan Lilacine Bushbrown & 0 & 0 & 0 & 0 & 0 & 0 & 1 & 1 \\
\hline 239 & Mycalesis intermedia & Intermediate Bushbrown & 0 & 2 & 0 & 0 & 0 & 1 & 0 & 3 \\
\hline 240 & Mycalesis malsarida & Plain Bushbrown & 0 & 4 & 0 & 3 & 0 & 0 & 0 & 7 \\
\hline 241 & Mycalesis mineus mineus & Chinese Dark-branded Bushbrown & 1 & 2 & 0 & 0 & 0 & 0 & 0 & 3 \\
\hline 242 & Mycalesis perseus blasius & Himalayan Common Bushbrown & 0 & 2 & 0 & 1 & 1 & 0 & 1 & 5 \\
\hline 243 & Mycalesis sp. & Bushbrown sp. & 10 & 0 & 1 & 0 & 5 & 0 & 0 & 16 \\
\hline 244 & Mycalesis visala visala & Indian Long-branded Bushbrown & 0 & 1 & 0 & 0 & 0 & 0 & 0 & 1 \\
\hline 245 & Orsotriaena medus medus & Oriental Medus Brown & 2 & 4 & 2 & 2 & 1 & 0 & 0 & 11 \\
\hline 246 & Thaumantis diores diores & Assam Jungleglory & 1 & 2 & 0 & 1 & 0 & 2 & 0 & 6 \\
\hline 247 & Ypthima baldus satpura & Satpuda Common Five-ring & 9 & 49 & 15 & 7 & 1 & 7 & 1 & 89 \\
\hline 248 & Ypthima huebneri & Common Four-ring & 1 & 0 & 10 & 0 & 0 & 0 & 0 & 11 \\
\hline
\end{tabular}

Family Papilionidae, Subfamily Papilioninae:

\begin{tabular}{|c|c|c|c|c|c|c|c|c|c|c|}
\hline 249 & Atrophaneura varuna astorion & Sylhet Common Batwing & 3 & 12 & 7 & 1 & 0 & 0 & 0 & 23 \\
\hline 250 & Byasa sp. & Windmill sp. & 0 & 1 & 0 & 0 & 0 & 0 & 0 & 1 \\
\hline 251 & Graphium agamemnon agamemnon & Oriental Tailed Jay & 5 & 2 & 2 & 0 & 2 & 0 & 0 & 11 \\
\hline 252 & Graphium agetes agetes & Assam Four-bar Swordtail & 0 & 0 & 5 & 0 & 0 & 0 & 0 & 5 \\
\hline 253 & Graphium antiphates pompilius & Indo-Chinese Five-bar Swordtail & 7 & 0 & 5 & 0 & 1 & 0 & 0 & 13 \\
\hline 254 & Graphium aristeus anticrates & Assam Chain Swordtail & 0 & 0 & 0 & 0 & 1 & 0 & 0 & 1 \\
\hline 255 & Graphium cloanthus cloanthus & Himalayan Glassy Bluebottle & 0 & 1 & 0 & 0 & 0 & 0 & 1 & 2 \\
\hline 256 & Graphium doson axion & Himalayan Common Jay & 17 & 0 & 13 & 0 & 5 & 0 & 0 & 35 \\
\hline 257 & Graphium eurypylus cheronus & Indo-Chinese Great Jay & 0 & 1 & 3 & 0 & 0 & 0 & 0 & 4 \\
\hline
\end{tabular}




\begin{tabular}{|c|c|c|c|c|c|c|c|c|c|c|}
\hline No & Scientific name & English name & $\begin{array}{l}\text { BNP } \\
\text { SUM }\end{array}$ & $\begin{array}{l}\text { BNP } \\
\text { WIN }\end{array}$ & $\begin{array}{l}\text { BRF } \\
\text { SUM }\end{array}$ & $\begin{array}{l}\text { BRF } \\
\text { WIN }\end{array}$ & $\begin{array}{l}\text { SIJU } \\
\text { SUM }\end{array}$ & $\begin{array}{l}\text { SIJU } \\
\text { WIN }\end{array}$ & $\begin{array}{l}\text { NNP } \\
\text { WIN }\end{array}$ & Total \\
\hline 258 & Graphium macareus lioneli & Khasi Lesser Zebra & 0 & 0 & 1 & 0 & 0 & 0 & 0 & 1 \\
\hline 259 & Graphium sarpedon sarpedon & Oriental Common Bluebottle & 10 & 2 & 4 & 0 & 1 & 0 & 0 & 17 \\
\hline 260 & Graphium xenocles xenocles & Sylhet Great Zebra & 4 & 0 & 1 & 0 & 1 & 0 & 0 & 6 \\
\hline 261 & Lamproptera curius curius & Thai White Dragontail & 3 & 7 & 1 & 0 & 0 & 0 & 0 & 11 \\
\hline 262 & Losaria coon cacharensis & Cachar Common Clubtail & 0 & 0 & 1 & 0 & 0 & 0 & 0 & 1 \\
\hline 263 & Meandrusa payeni evan & Sikkim Yellow Gorgon & 0 & 1 & 0 & 0 & 0 & 0 & 0 & 1 \\
\hline 264 & Pachliopta aristolochiae aristolochiae & Indian Common Rose & 17 & 6 & 43 & 1 & 2 & 0 & 0 & 69 \\
\hline 265 & Papilio alcmenor alcmenor & Khasi Redbreast & 0 & 1 & 0 & 0 & 0 & 0 & 0 & 1 \\
\hline 266 & Papilio bianor gladiator & Indo-Chinese Common Peacock & 0 & 0 & 1 & 0 & 0 & 0 & 0 & 1 \\
\hline 267 & Papilio castor castor & Khasi Common Raven & 1 & 0 & 9 & 0 & 7 & 0 & 0 & 17 \\
\hline 268 & Papilio clytia clytia & Oriental Common Mime & 1 & 3 & 3 & 0 & 1 & 0 & 0 & 8 \\
\hline 269 & Papilio demoleus demoleus & Northern Lime Butterfly & 6 & 0 & 1 & 1 & 1 & 0 & 0 & 9 \\
\hline 270 & Papilio helenus helenus & Oriental Red Helen & 11 & 8 & 10 & 0 & 1 & 5 & 3 & 38 \\
\hline 271 & Papilio memnon agenor & Continental Great Mormon & 8 & 9 & 7 & 4 & 0 & 0 & 1 & 29 \\
\hline 272 & Papilio nephelus chaon & Assam Yellow Helen & 13 & 1 & 18 & 0 & 1 & 0 & 1 & 34 \\
\hline 273 & Papilio paradoxa telearchus & Khasi Great Blue Mime & 0 & 0 & 1 & 0 & 0 & 0 & 0 & 1 \\
\hline 274 & Papilio paris paris & Chinese Paris Peacock & 1 & 0 & 1 & 0 & 0 & 0 & 10 & 12 \\
\hline 275 & Papilio polytes romulus & Indian Common Mormon & 31 & 13 & 17 & 0 & 5 & 0 & 3 & 69 \\
\hline 276 & Papilio protenor euprotenor & East Himalayan Spangle & 2 & 0 & 0 & 0 & 0 & 0 & 0 & 2 \\
\hline 277 & Troides helena cerberus & Khasi Common Birdwing & 4 & 5 & 4 & 0 & 0 & 0 & 0 & 13 \\
\hline
\end{tabular}

Family Pieridae, Subfamily Coliadinae:

\begin{tabular}{|c|c|c|c|c|c|c|c|c|c|c|}
\hline 278 & Catopsilia pomona pomona & Oriental Common Emigrant & 13 & 7 & 24 & 0 & 0 & 0 & 0 & 44 \\
\hline 279 & Catopsilia pyranthe pyranthe & Oriental Mottled Emigrant & 0 & 0 & 0 & 1 & 0 & 0 & 0 & 1 \\
\hline 280 & Dercas verhuelli doubledayi & Indo-Chinese Tailed Sulphur & 5 & 0 & 0 & 0 & 0 & 0 & 0 & 5 \\
\hline 281 & Eurema andersonii jordani & Sikkim One-spot Grass Yellow & 0 & 15 & 0 & 0 & 0 & 2 & 0 & 17 \\
\hline 282 & Eurema blanda silhetana & Sylhet Three-spot Grass Yellow & 10 & 52 & 3 & 1 & 0 & 2 & 0 & 68 \\
\hline 283 & Eurema hecabe hecabe & Oriental Common Grass Yellow & 28 & 11 & 12 & 2 & 1 & 0 & 1 & 55 \\
\hline 284 & Gandaca harina assamica & Assam Tree Yellow & 6 & 10 & 6 & 0 & 3 & 0 & 0 & 25 \\
\hline
\end{tabular}

Family Pieridae, Subfamily Pierinae:

\begin{tabular}{|c|c|c|c|c|c|c|c|c|c|c|}
\hline 285 & Appias albina darada & Indian Common Albatross & 2 & 2 & 0 & 0 & 0 & 0 & 0 & 4 \\
\hline 286 & Appias indra indra & Himalayan Plain Puffin & 21 & 0 & 1 & 0 & 1 & 0 & 0 & 23 \\
\hline 287 & Appias lalage lalage & Himalayan Spot Puffin & 0 & 0 & 0 & 0 & 0 & 0 & 1 & 1 \\
\hline 288 & Appias lyncida eleonora & Indo-Chinese Chocolate Albatross & 23 & 48 & 21 & 7 & 0 & 0 & 0 & 99 \\
\hline 289 & Appias olferna & Eastern Striped Albatross & 1 & 0 & 3 & 0 & 0 & 0 & 0 & 4 \\
\hline 290 & Cepora nadina nadina & Khasi Lesser Gull & 2 & 10 & 0 & 0 & 0 & 1 & 0 & 13 \\
\hline
\end{tabular}




\begin{tabular}{|c|c|c|c|c|c|c|c|c|c|c|}
\hline No & Scientific name & English name & $\begin{array}{l}\text { BNP } \\
\text { SUM }\end{array}$ & $\begin{array}{l}\text { BNP } \\
\text { WIN }\end{array}$ & $\begin{array}{l}\text { BRF } \\
\text { SUM }\end{array}$ & $\begin{array}{l}\text { BRF } \\
\text { WIN }\end{array}$ & $\begin{array}{l}\text { SIJU } \\
\text { SUM }\end{array}$ & $\begin{array}{l}\text { SIJU } \\
\text { WIN }\end{array}$ & $\begin{array}{l}\text { NNP } \\
\text { WIN }\end{array}$ & Total \\
\hline 291 & Cepora nerissa nerissa & Himalayan Common Gull & 2 & 0 & 0 & 0 & 0 & 0 & 0 & 2 \\
\hline 292 & Delias agostina agostina & Sikkim Yellow Jezebel & 0 & 0 & 0 & 1 & 0 & 0 & 0 & 1 \\
\hline 293 & Delias belladonna lugens & Lushai Hill Jezebel & 1 & 0 & 0 & 1 & 0 & 0 & 0 & 2 \\
\hline 294 & Delias descombesi descombesi & Vietnamese Red-spot Jezebel & 0 & 7 & 0 & 0 & 0 & 0 & 3 & 10 \\
\hline 295 & Delias hyparete indica & Himalayan Painted Jezebel & 0 & 1 & 0 & 2 & 0 & 0 & 0 & 3 \\
\hline 296 & Delias pasithoe pasithoe & Chinese Red-base Jezebel & 0 & 4 & 1 & 5 & 0 & 0 & 3 & 13 \\
\hline 297 & Hebomoia glaucippe glaucippe & Oriental Great Orange-tip & 0 & 1 & 7 & 0 & 0 & 0 & 1 & 9 \\
\hline 298 & Ixias pyrene familiaris & East Himalayan Yellow Orange-tip & 3 & 3 & 0 & 0 & 0 & 0 & 1 & 7 \\
\hline 299 & Leptosia nina nina & Oriental Psyche & 0 & 2 & 0 & 0 & 0 & 0 & 1 & 3 \\
\hline 300 & Pareronia hippia & Indian Wanderer & 0 & 0 & 0 & 1 & 0 & 0 & 0 & 1 \\
\hline 301 & Pieris canidia indica & Indian Cabbage White & 1 & 0 & 0 & 0 & 0 & 0 & 0 & 1 \\
\hline
\end{tabular}

Family Riodinidae, Subfamily Nemeobiinae:

\begin{tabular}{|l|l|l|c|c|c|c|c|c|c|c|}
\hline 302 & Abisara bifasciata suffusa & Indian Double-banded Judy & 0 & 1 & 0 & 0 & 0 & 0 & 0 & 1 \\
\hline 303 & Abisara fylla & Dark Judy & 0 & 1 & 0 & 0 & 0 & 0 & 5 & 6 \\
\hline 304 & Abisara neophron neophron & Khasi Tailed Judy & 0 & 1 & 0 & 0 & 0 & 0 & 1 & 2 \\
\hline 305 & Dodona longicaudata & Long-tailed Punch & 0 & 1 & 0 & 0 & 0 & 0 & 0 & 1 \\
\hline 306 & Zemeros flegyas flegyas & Himalayan Punchinello & 2 & 25 & 2 & 3 & 0 & 0 & 30 & 62 \\
\hline & & Total & $\mathbf{9 5 8}$ & $\mathbf{9 6 1}$ & $\mathbf{1 , 0 6 7}$ & $\mathbf{2 7 1}$ & $\mathbf{1 7 4}$ & $\mathbf{1 2 0}$ & $\mathbf{2 5 4}$ & $\mathbf{3 , 8 0 4}$ \\
\hline
\end{tabular}

preservation of butterfly diversity in India.

\section{AN ANNOTATED CHECKLIST OF BUTTERFLIES OF THE GARO HILLS}

In addition to the summary and preliminary analyses of butterfly diversity presented above and in associated tables and figures, we now offer notes relevant to our sightings of butterflies in the Garo Hills. The numbers of individuals recorded for each species, season and general locality are given in Table 5 , which would be useful baseline quantitative data for any future work on these butterflies. Also mentioned for comparison is status of these and related species from the neighboring Khasi Hills.

\section{Family Hesperiidae (Skippers)} Subfamily Coeliadinae

1. Badamia exclamationis Fabricius, 1775 - Brown
Awl: Our six records were of individuals resting on the undersides of leaves along forest paths and forest streams and on one occasion, close to human habitation. This is a widely distributed species that has been reported as being common in the Khasi Hills (Cantlie 1956).

2. Bibasis sena sena Moore, 1865 - Indian Orangetail Awl: Our record of this species is based on a single specimen spotted late in the evening along the Simsang trail in Baghmara RF in April 2010. It has been reported from the Khasi Hills before (Cantlie 1956). It is legally protected in India under Schedule II of the Wildlife (Protection) Act (Anonymous 1997).

3. Burara oedipodea belesis Mabille, 1876 - Himalayan Branded Orange Awlet: A single specimen was photographed in May 2010 at Siju WS. This species has previously been reported from the Khasi Hills (Cantlie 1956).

4. Choaspes benjaminii japonica Murray, 1875 - Narrow Indian Awlking: A widespread species that 
Image 3. Butterflies of the Garo Hills

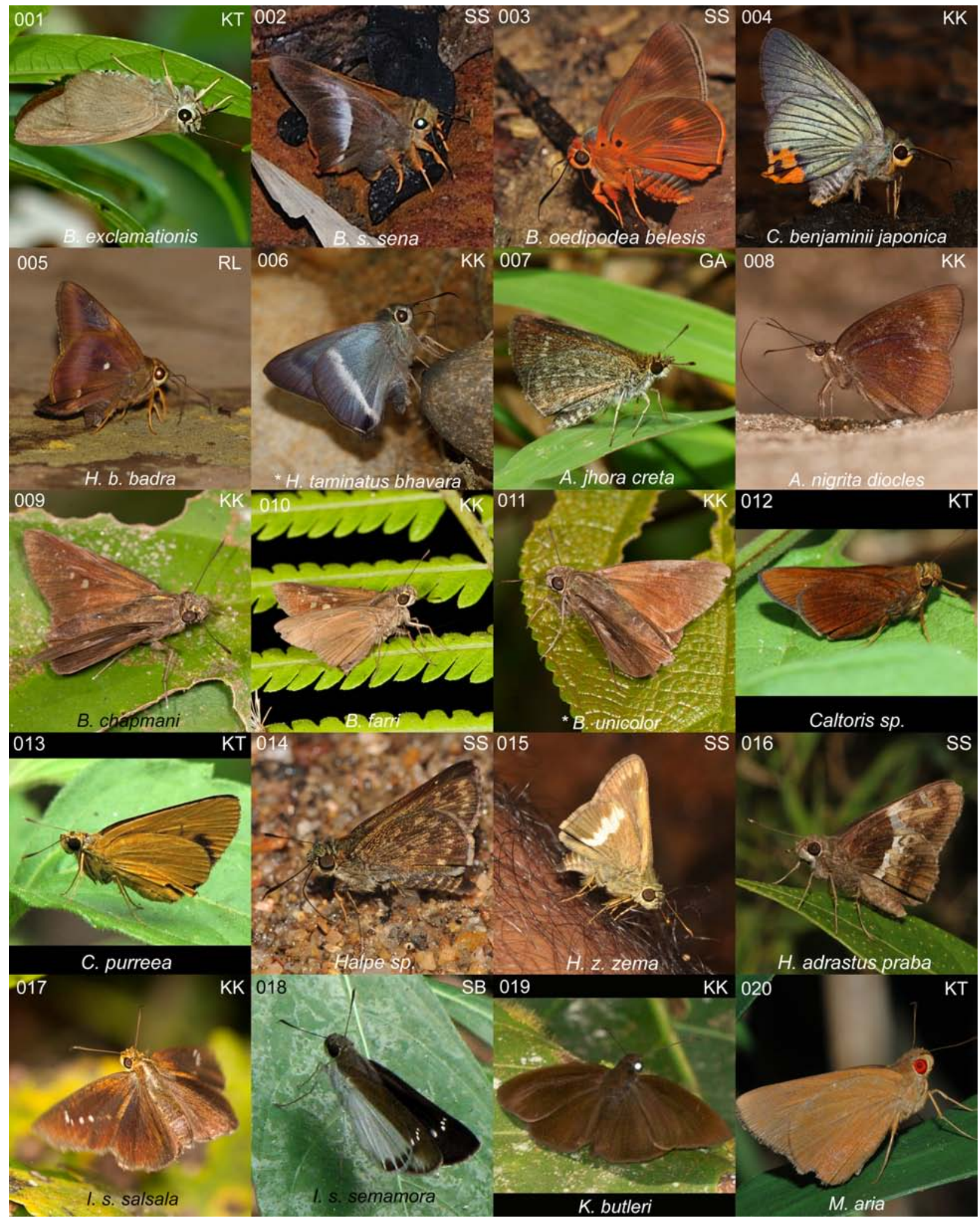




\section{Image 4. Butterflies of the Garo Hills}

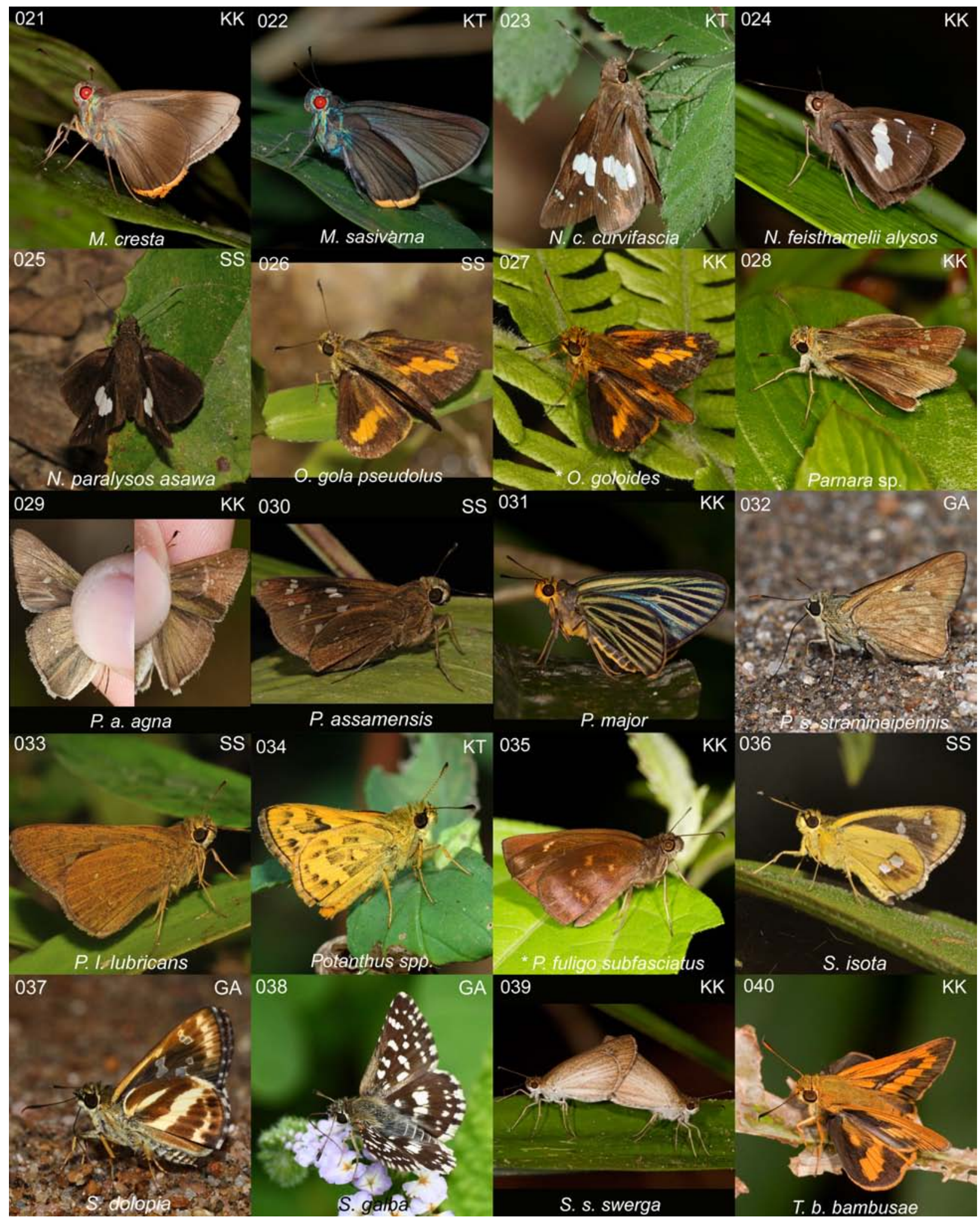




\section{Image 5. Butterflies of the Garo Hills}

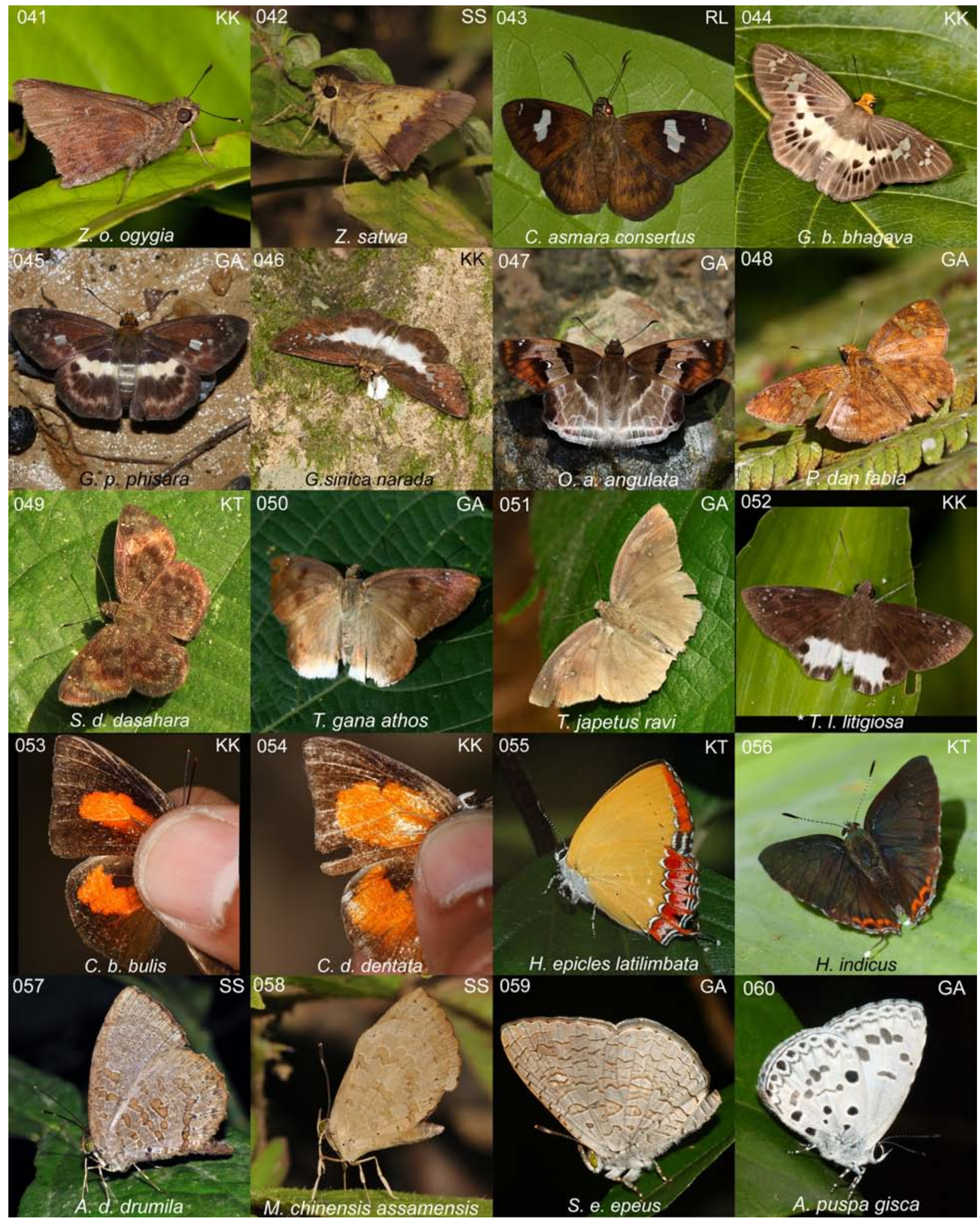


Image 6. Butterflies of the Garo Hills

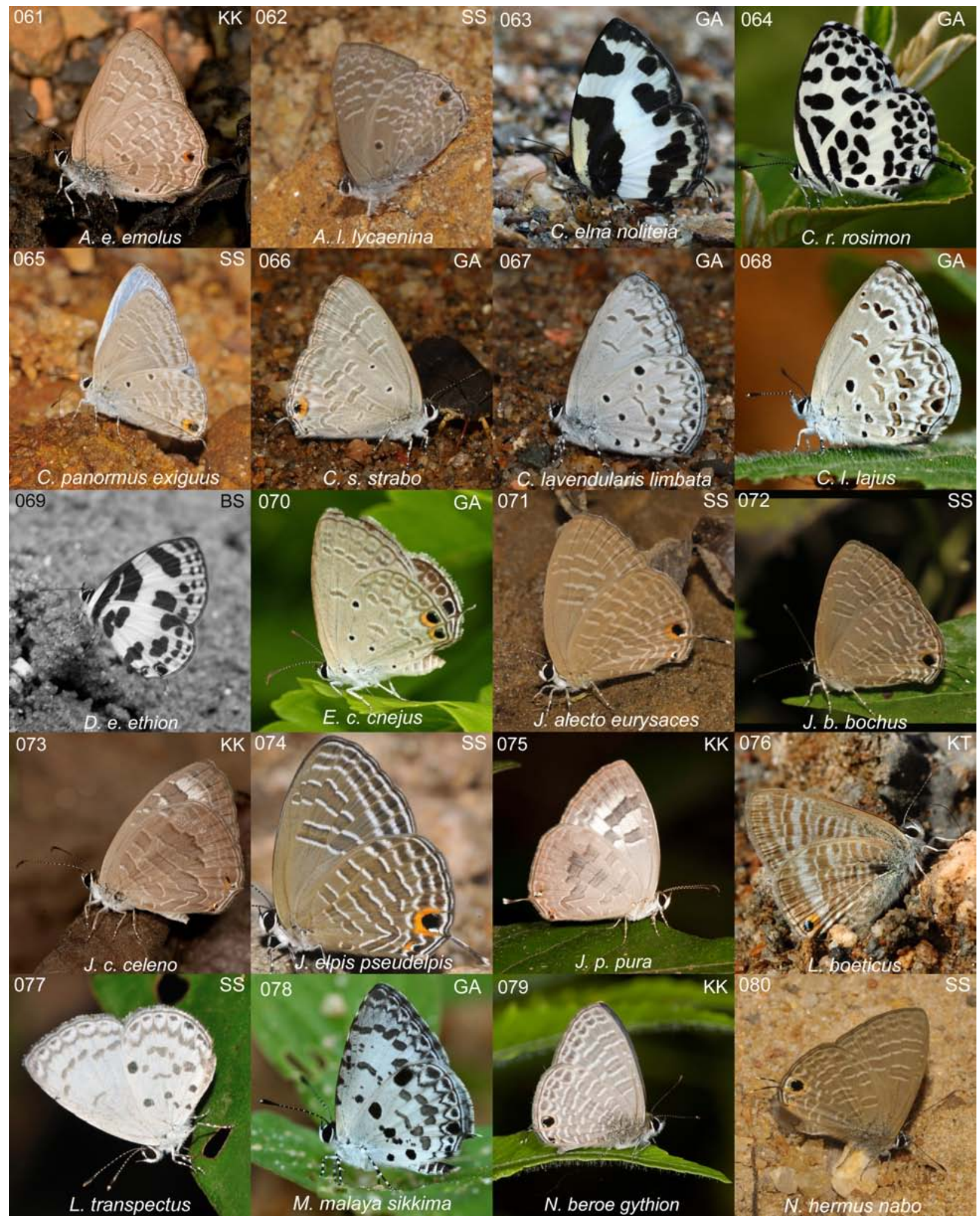




\section{Image 7. Butterflies of the Garo Hills}

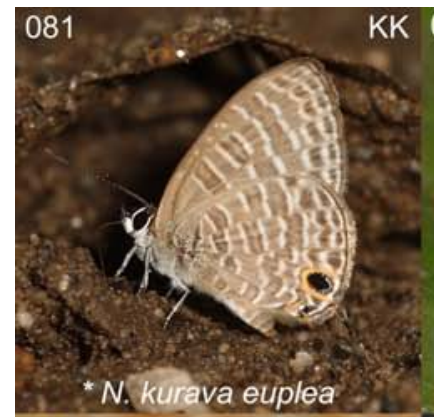

KK 082

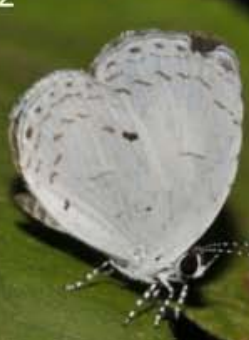

SS 083

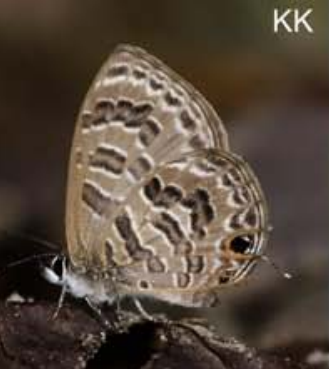

KK 084

KK

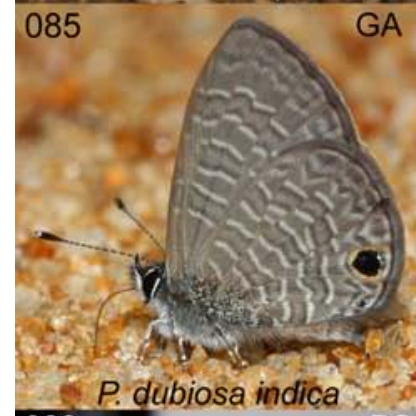

N. z. zalmora
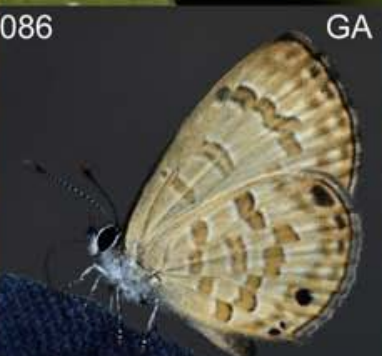

* $P$. aluta coelestis
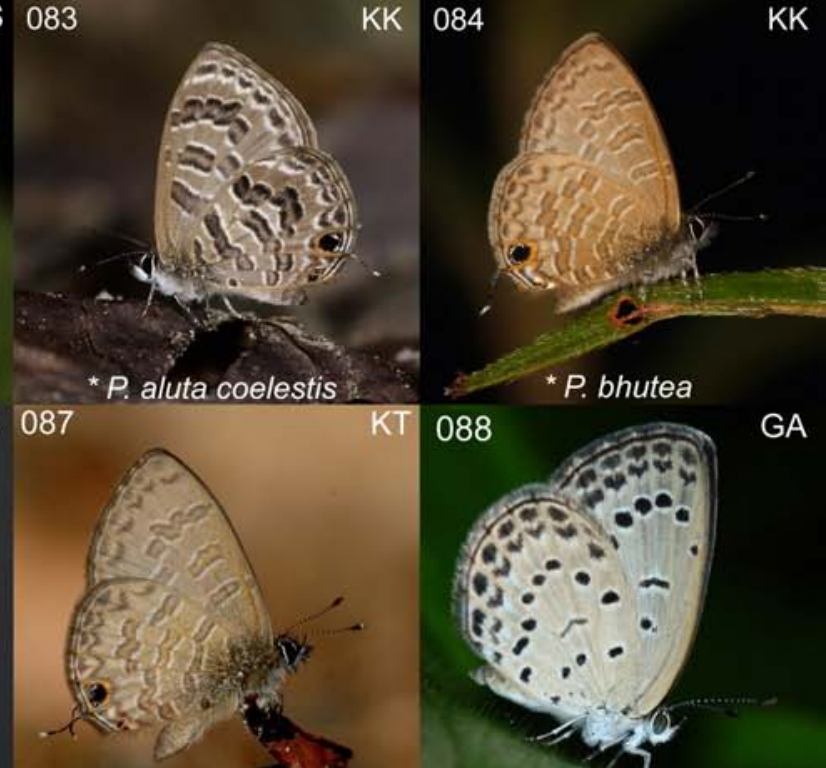

KT 088

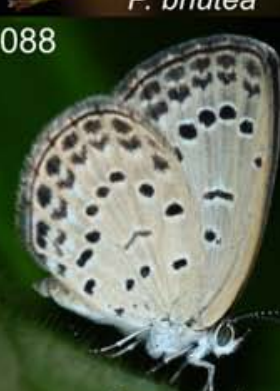

P. Iutea sivoka

\section{$P$ nora ardates}

P. m. maha
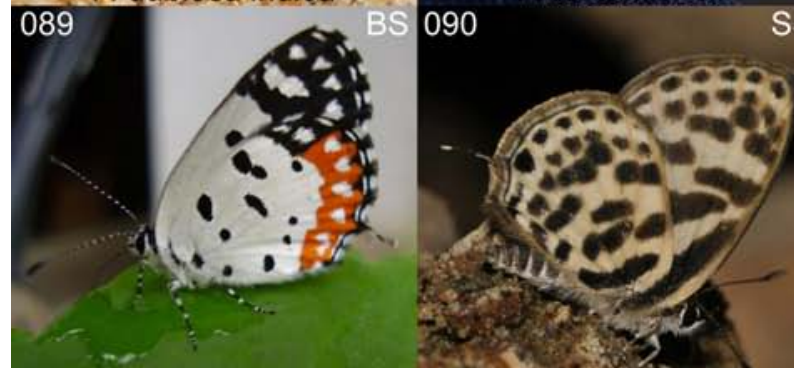

SS 091

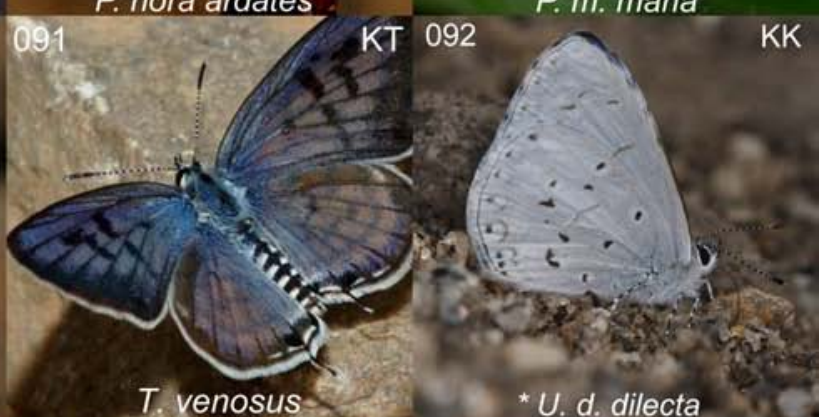

T. nyseus khasiana

QX..T. T. ananda

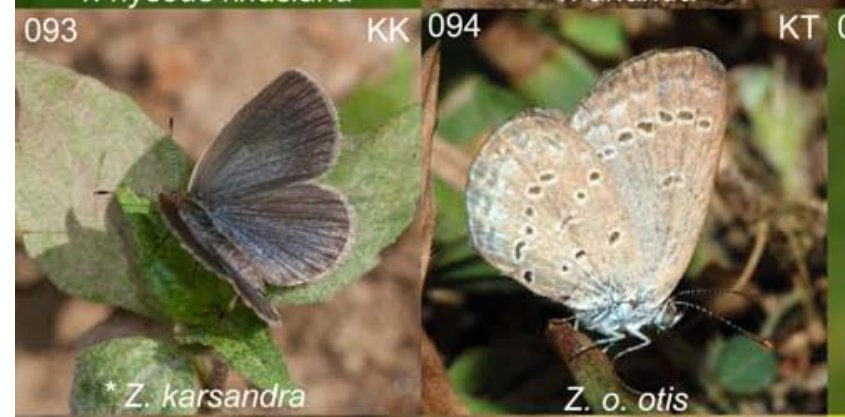

KT 095

T. venosus
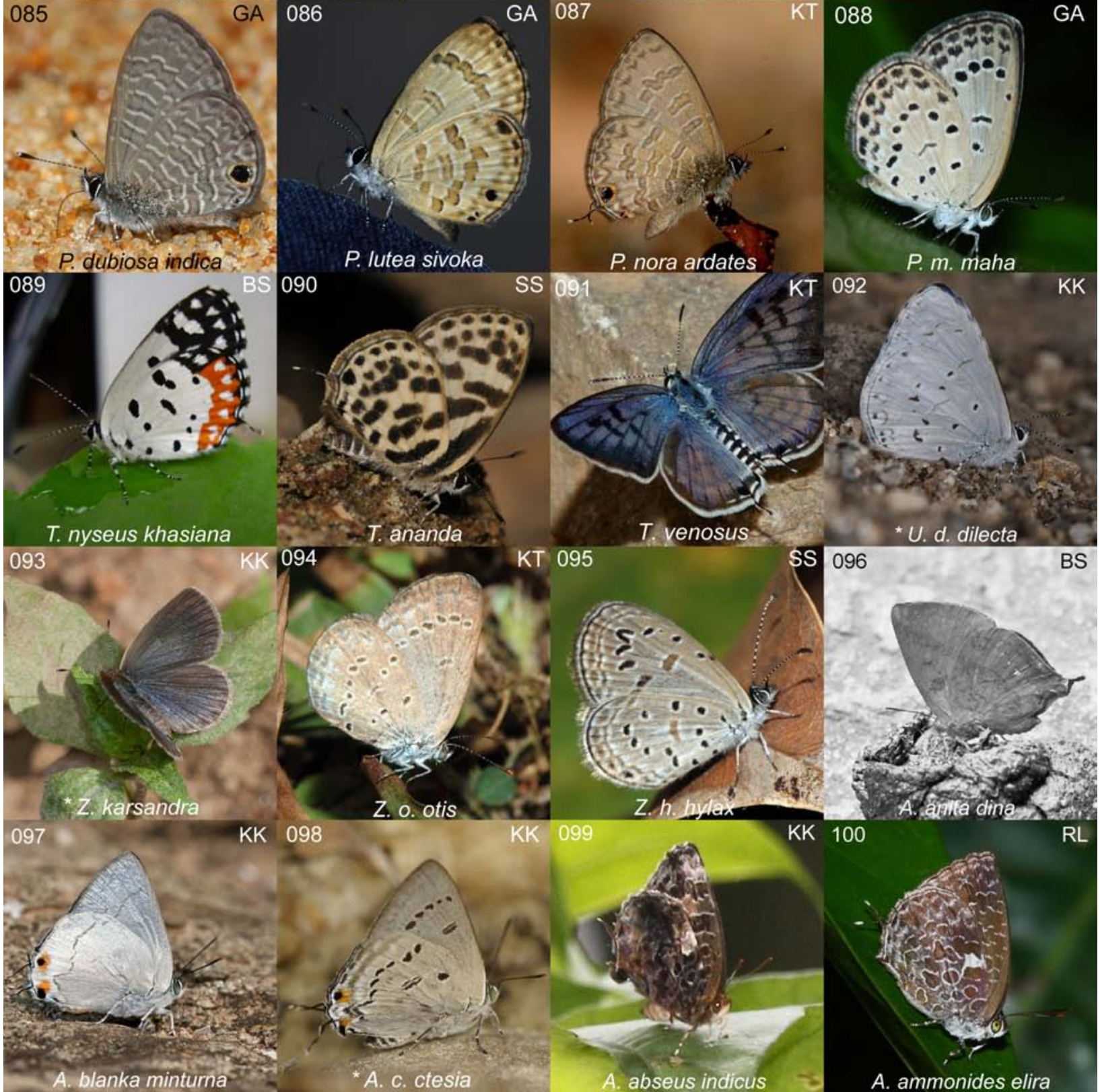

A. ammonides elira 
Image 8. Butterflies of the Garo Hills

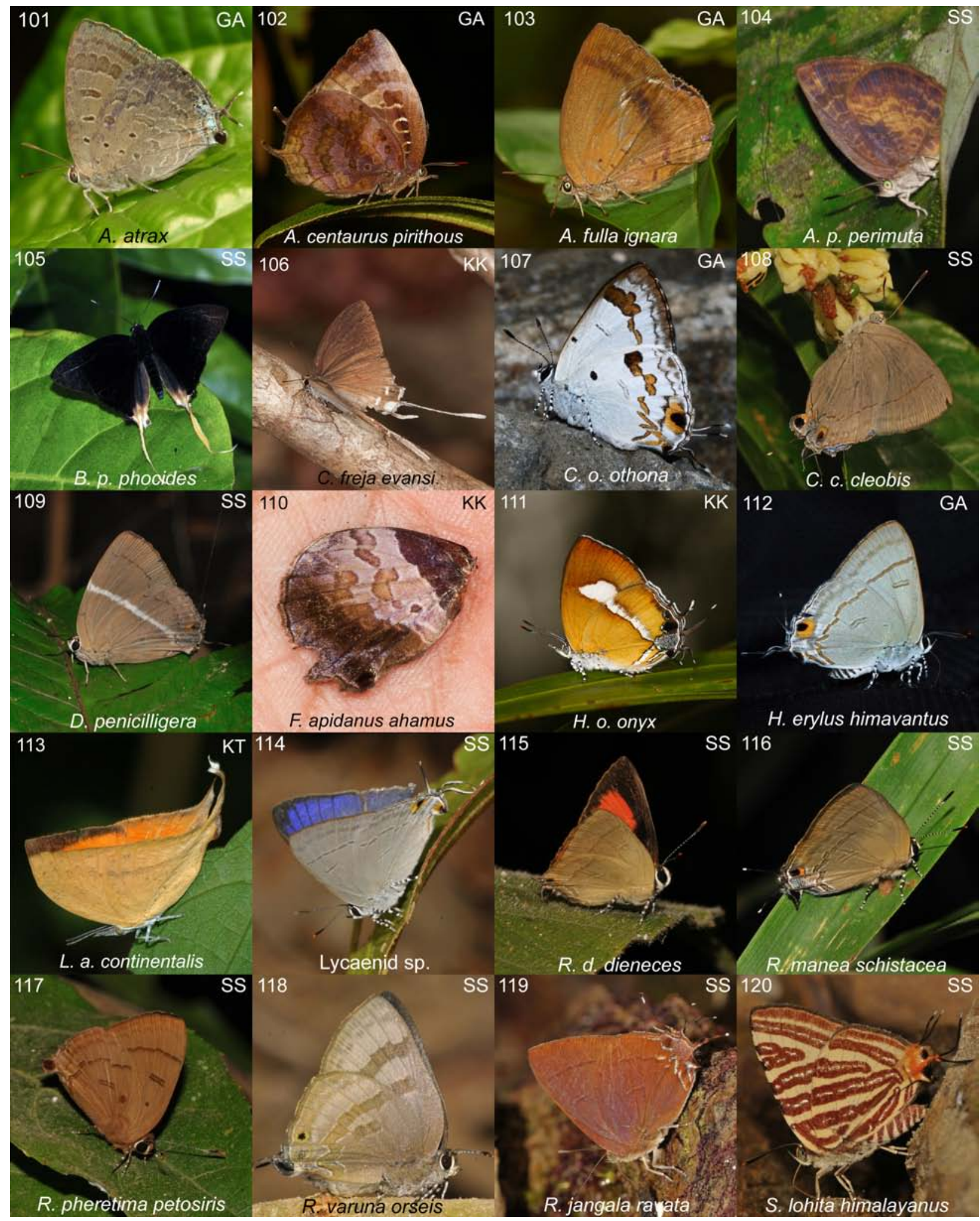


Image 9. Butterflies of the Garo Hills

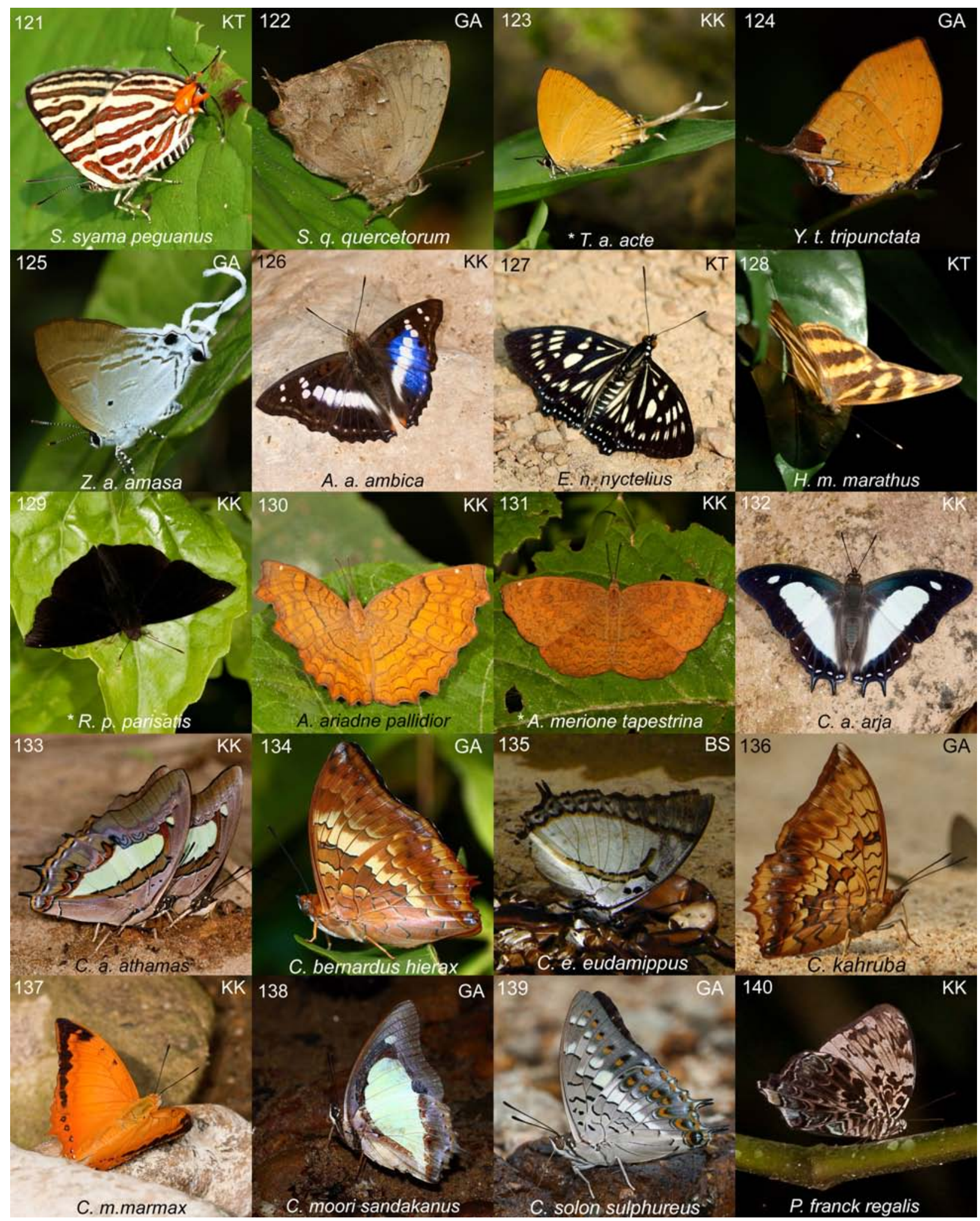


Image 10. Butterflies of the Garo Hills

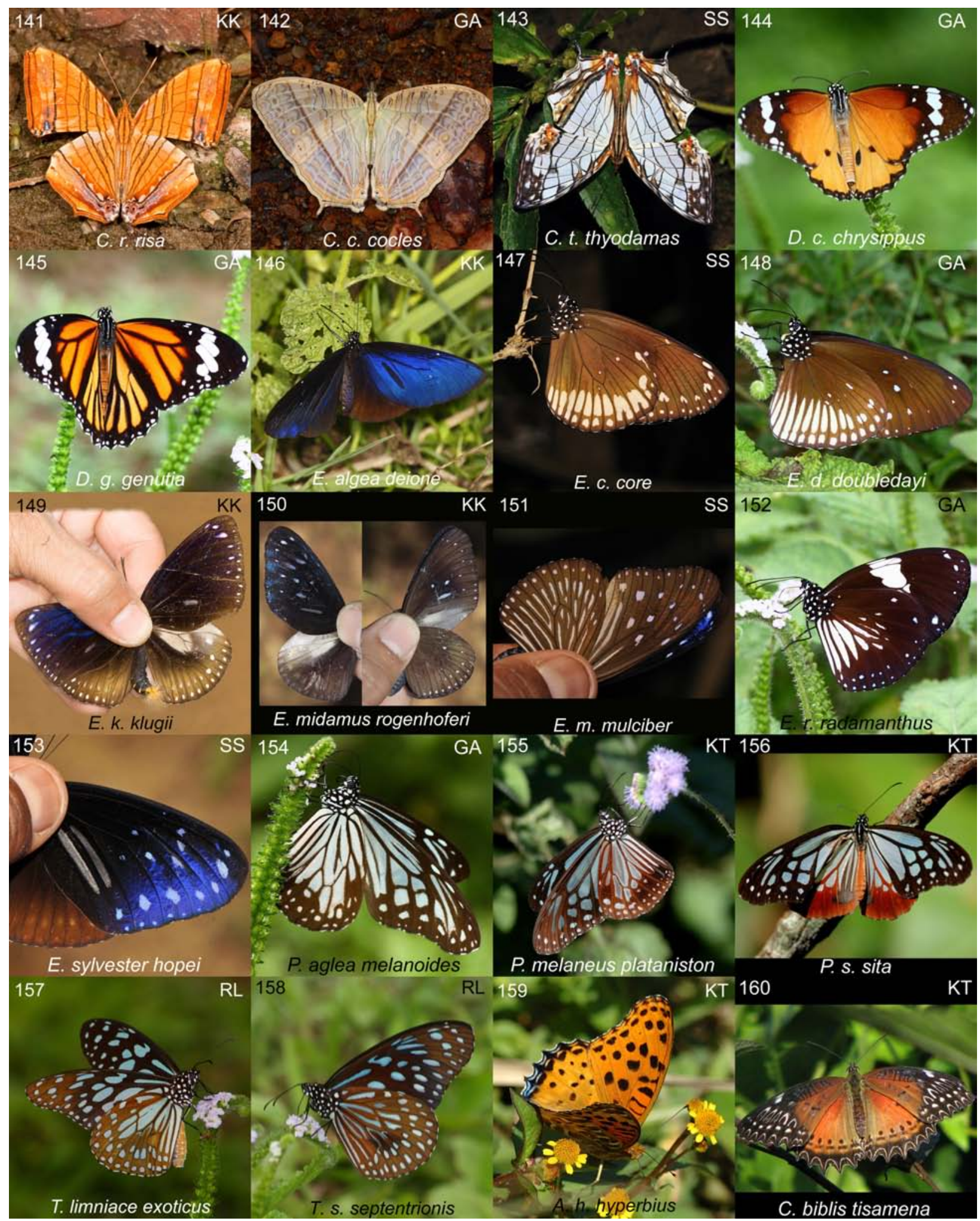


Image 11. Butterflies of the Garo Hills

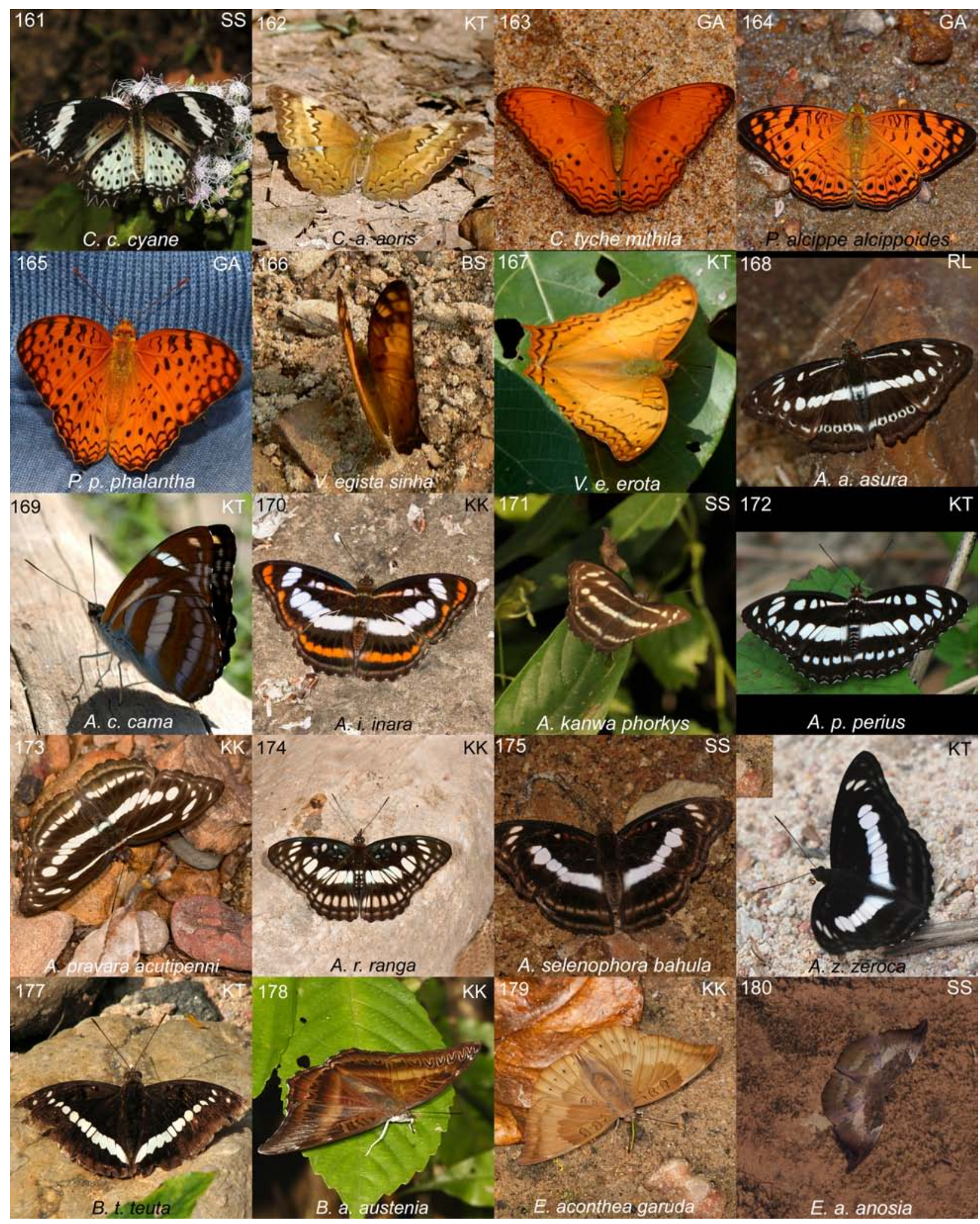


Image 12. Butterflies of the Garo Hills
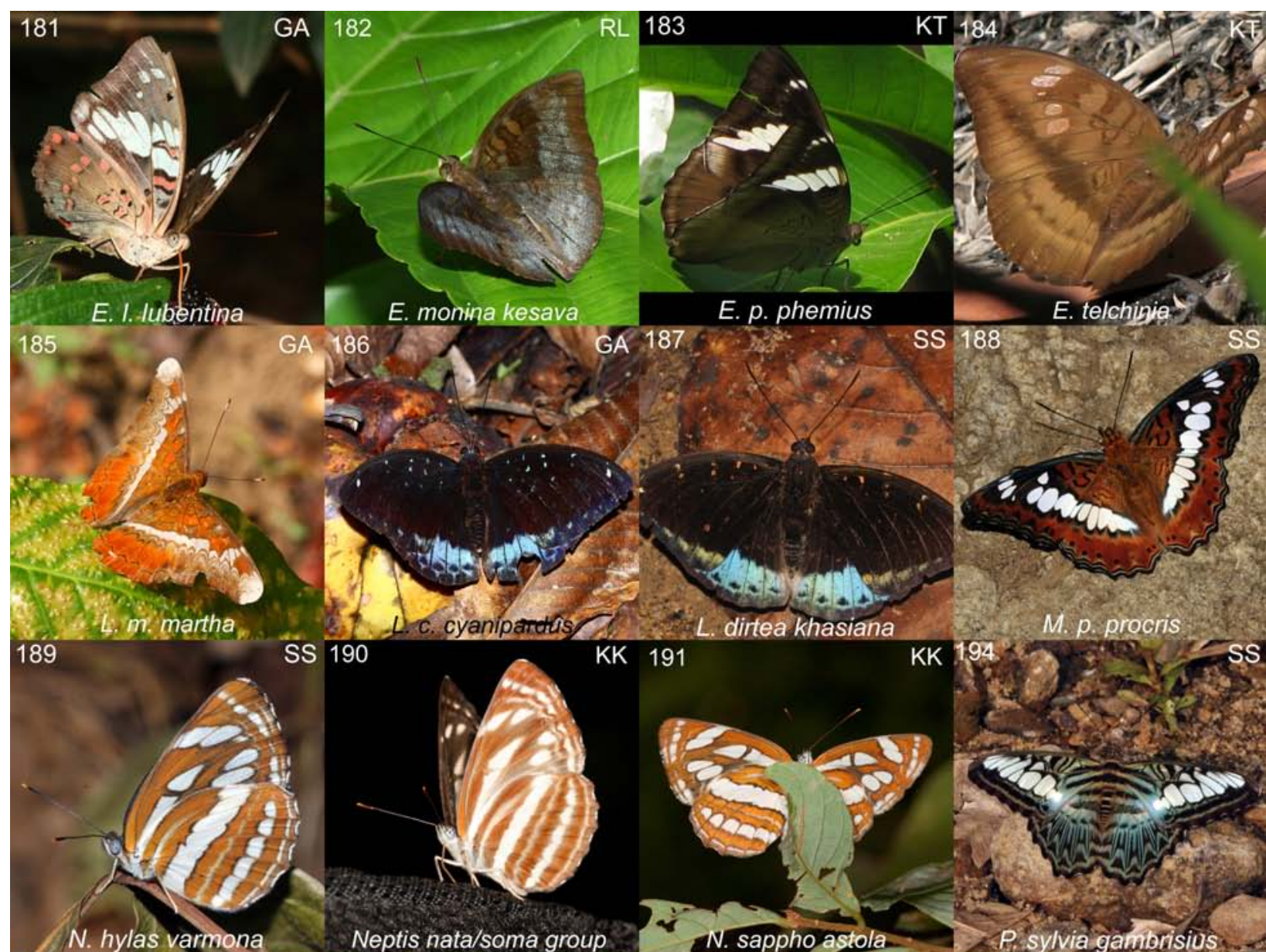

E. p. phemius
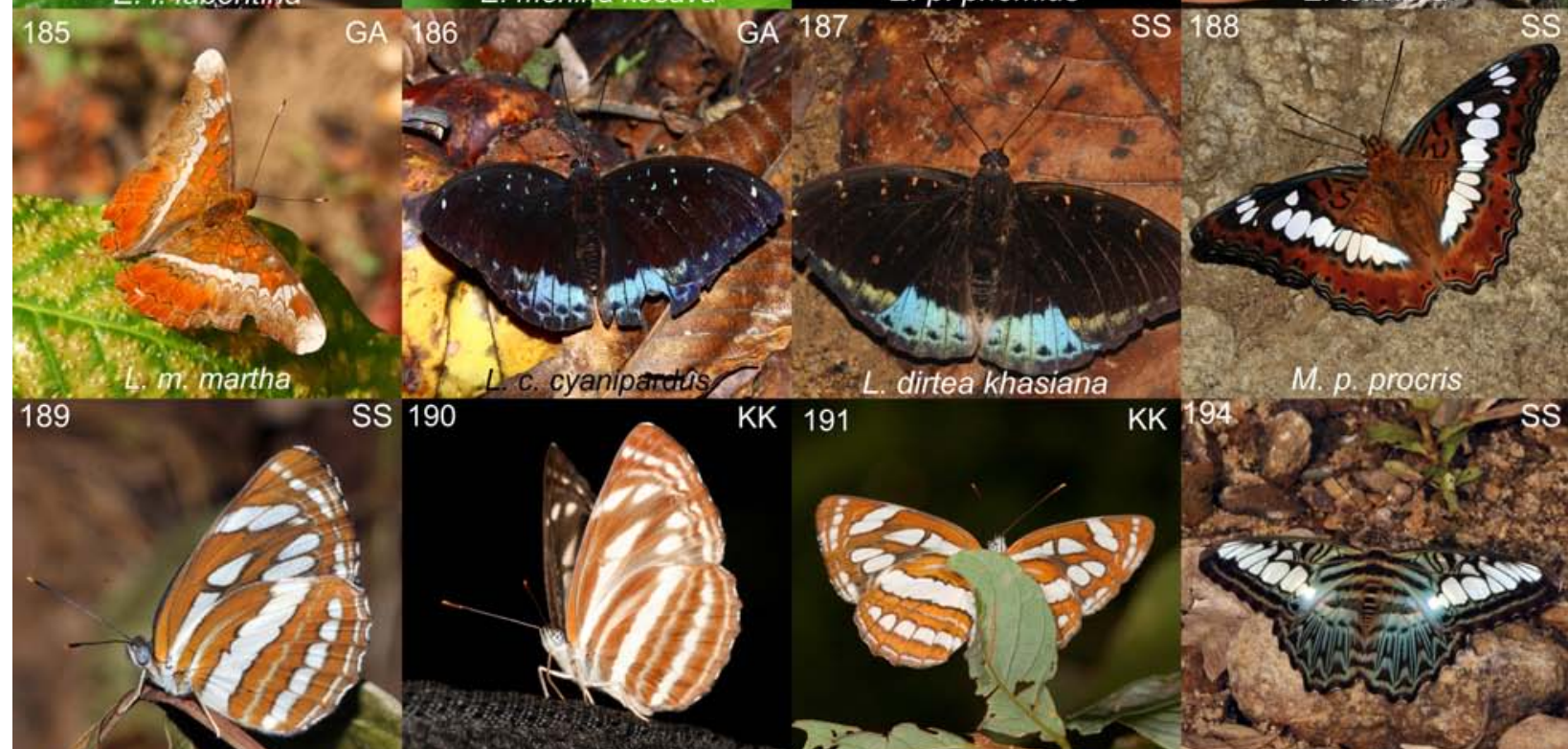

194 x 20 -

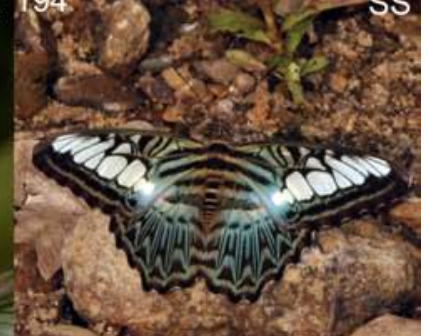

N. sappho astola - R. Sylvia gamionisios. 192

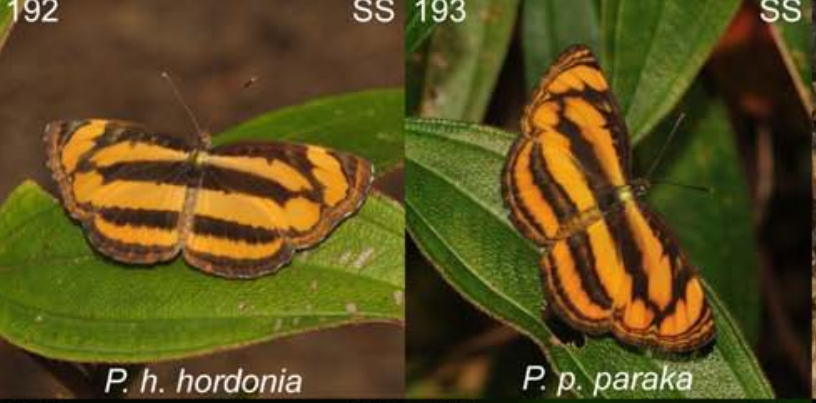

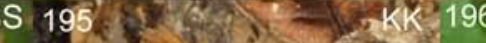

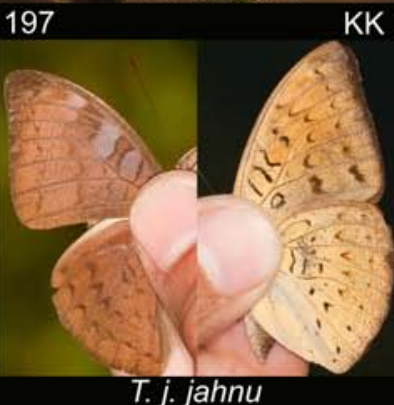

K 198

P. p. parak
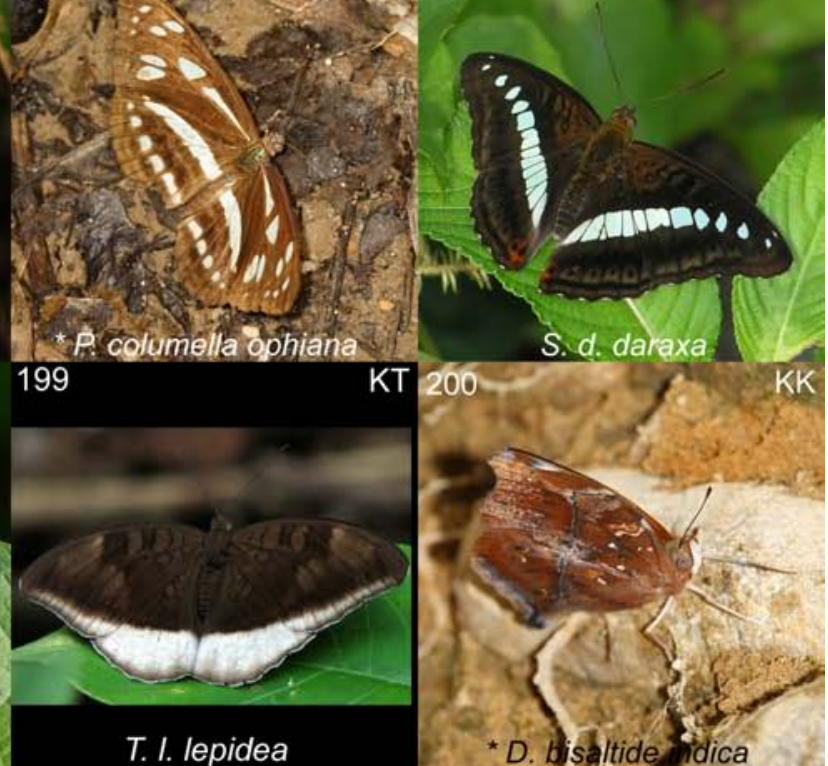
Image 13. Butterflies of the Garo Hills

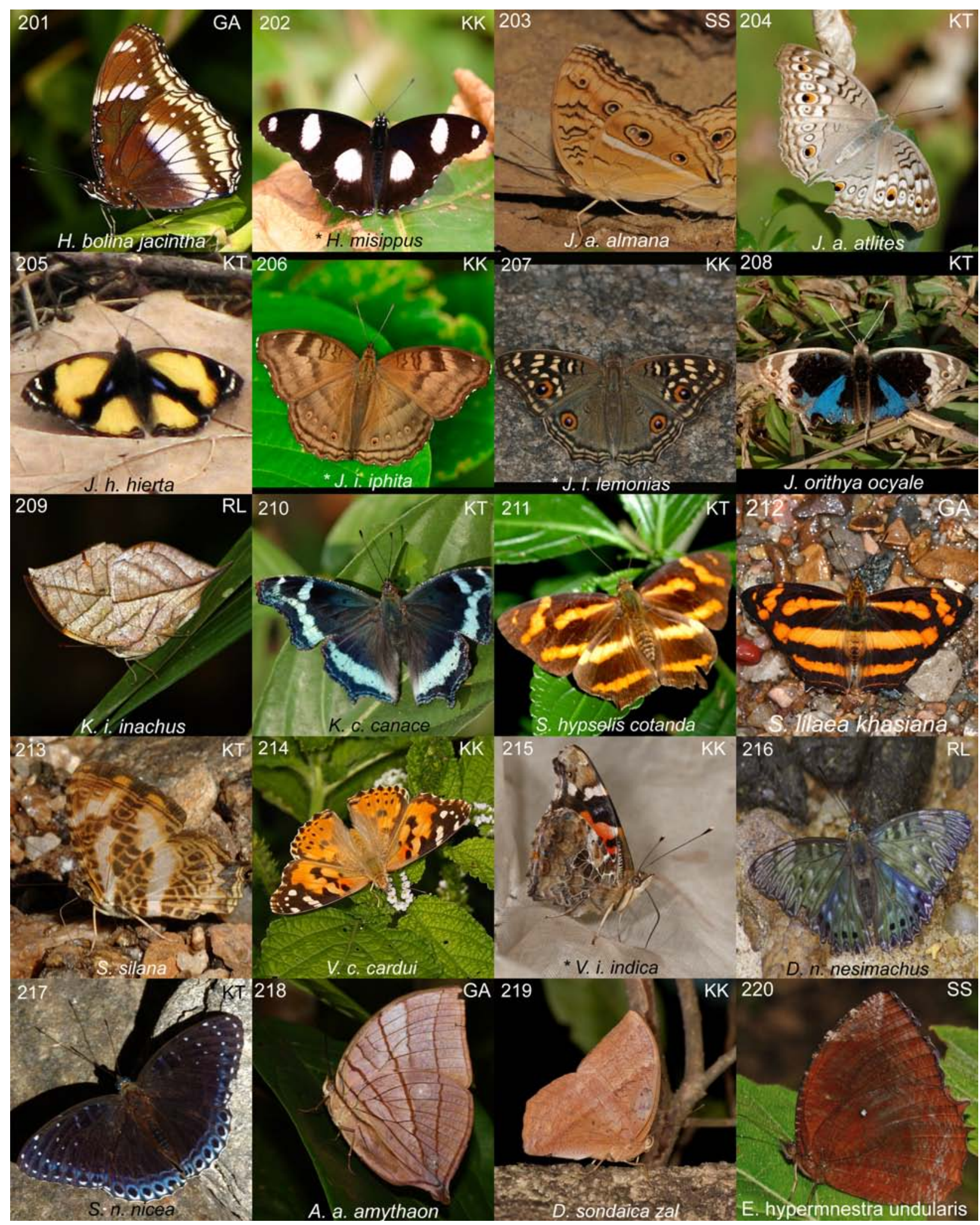




\section{Image 14. Butterflies of the Garo Hills}

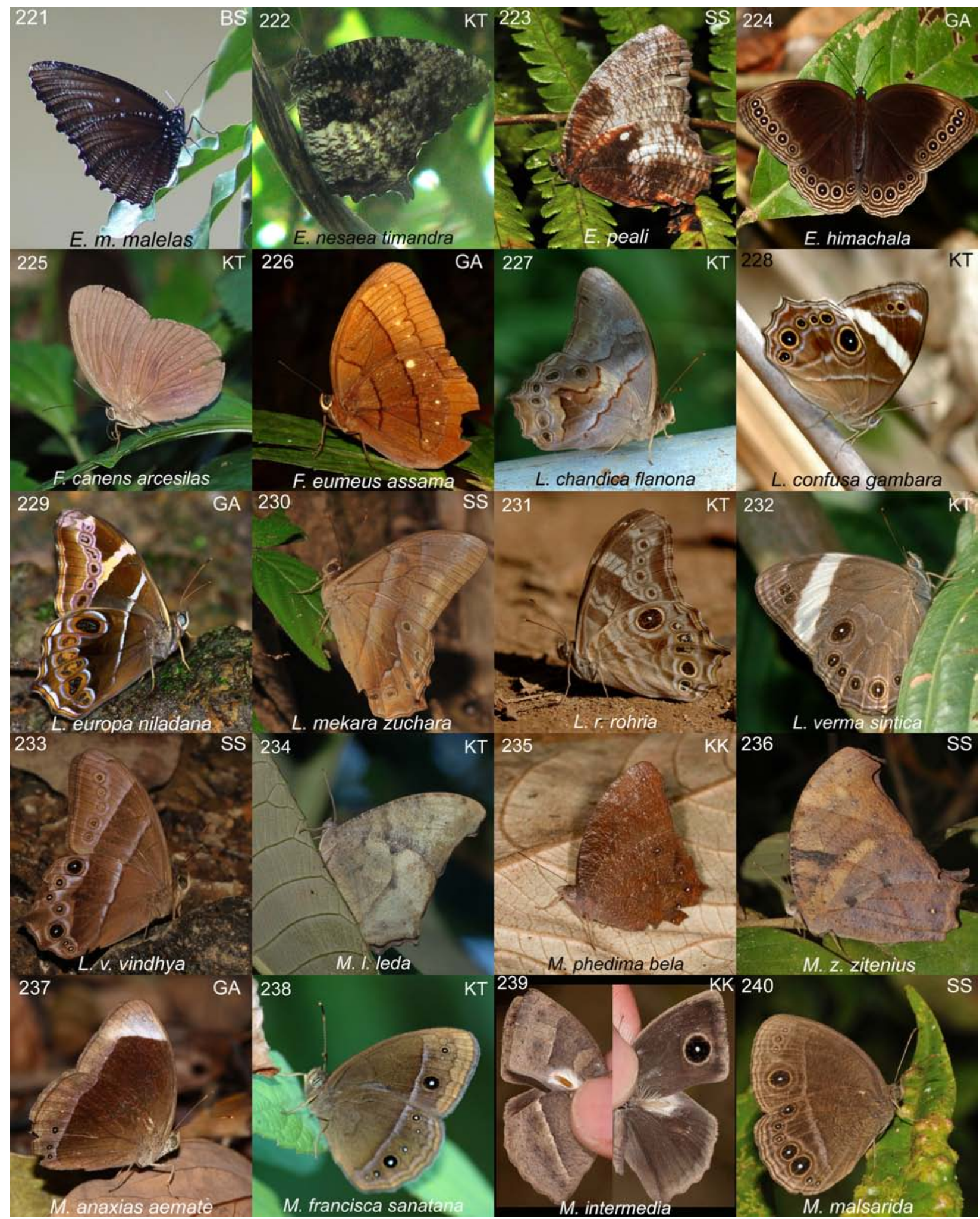


Image 15. Butterflies of the Garo Hills

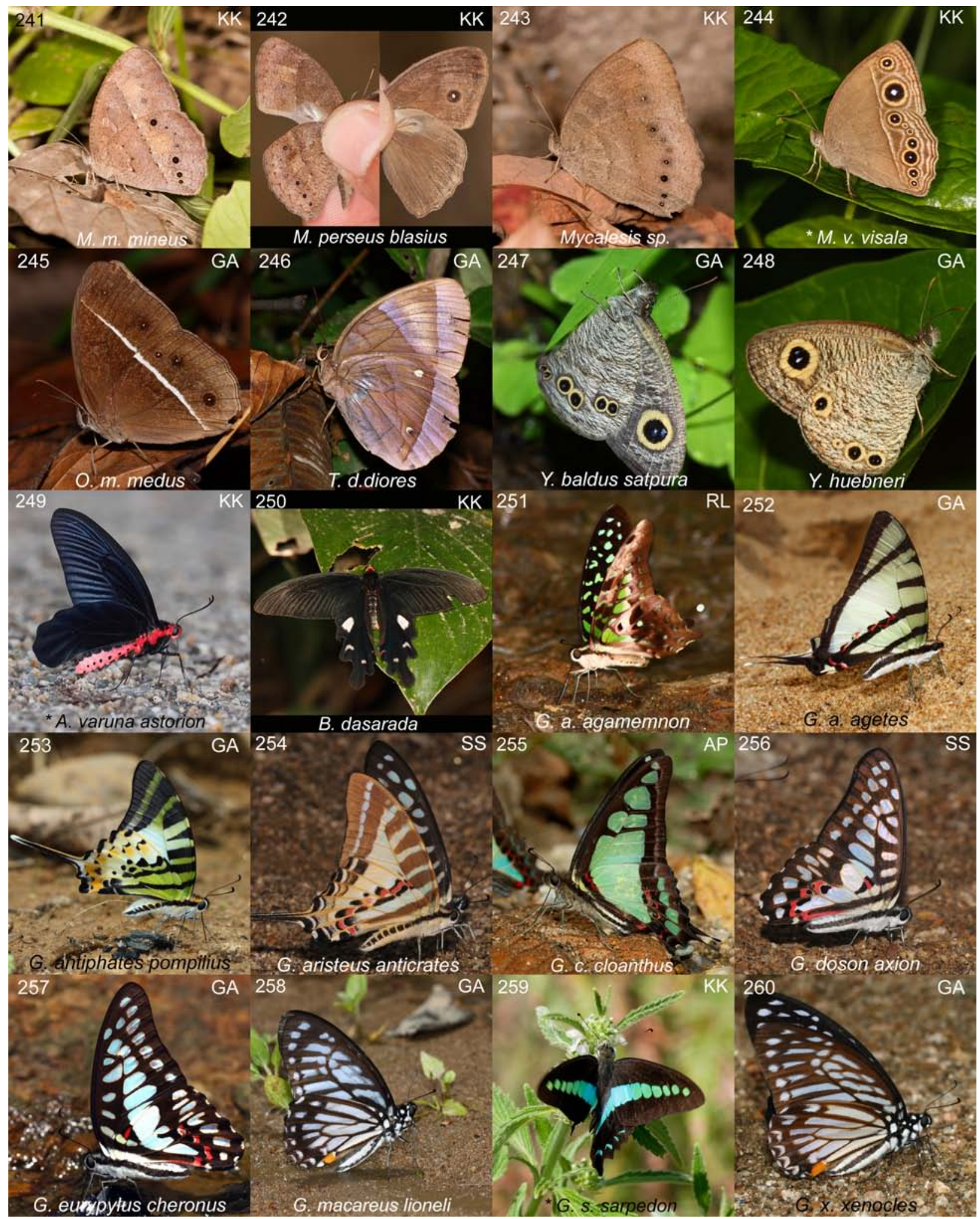


Image 16. Butterflies of the Garo Hills

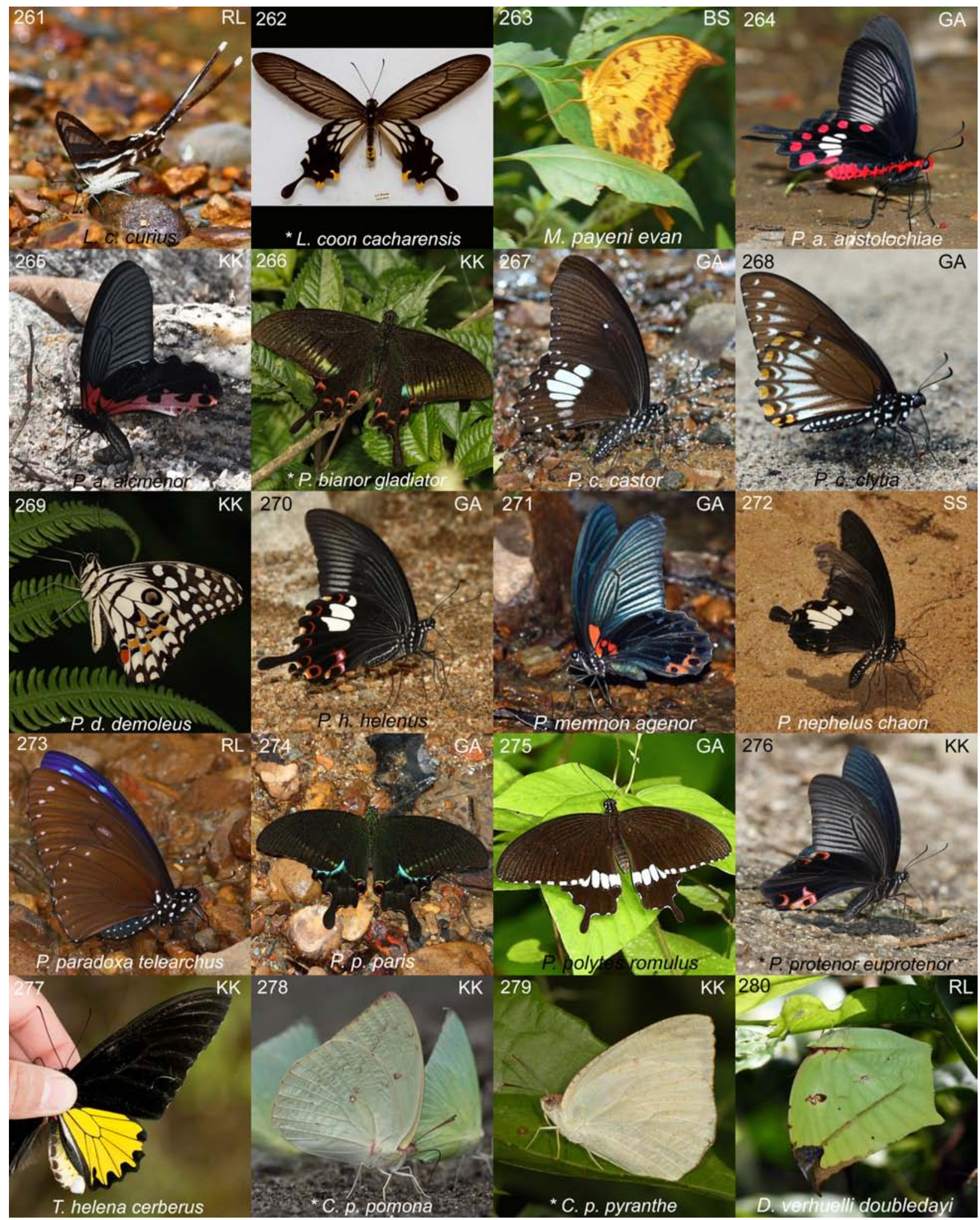




\section{Image 17. Butterflies of the Garo Hills}
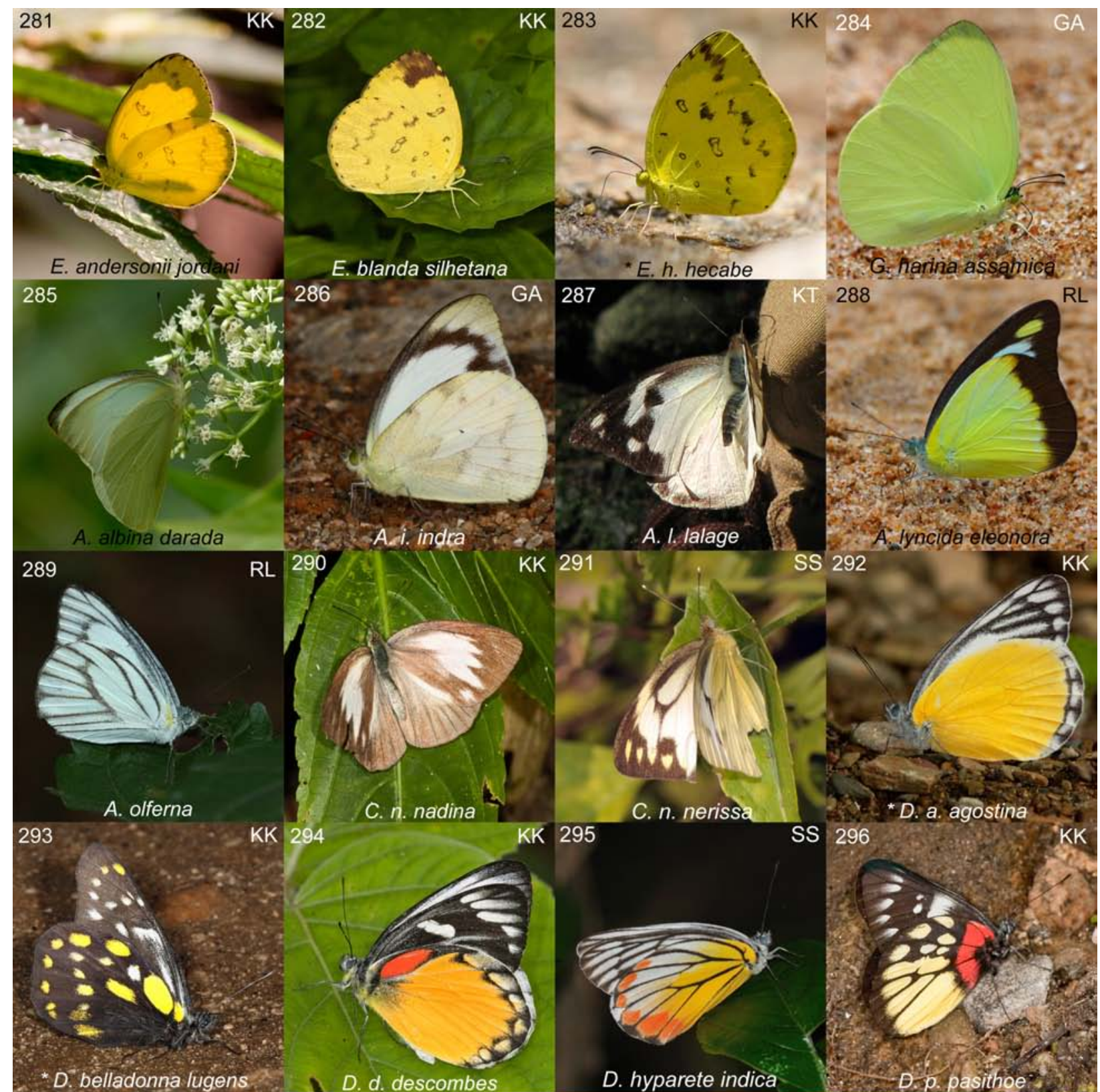

(2005
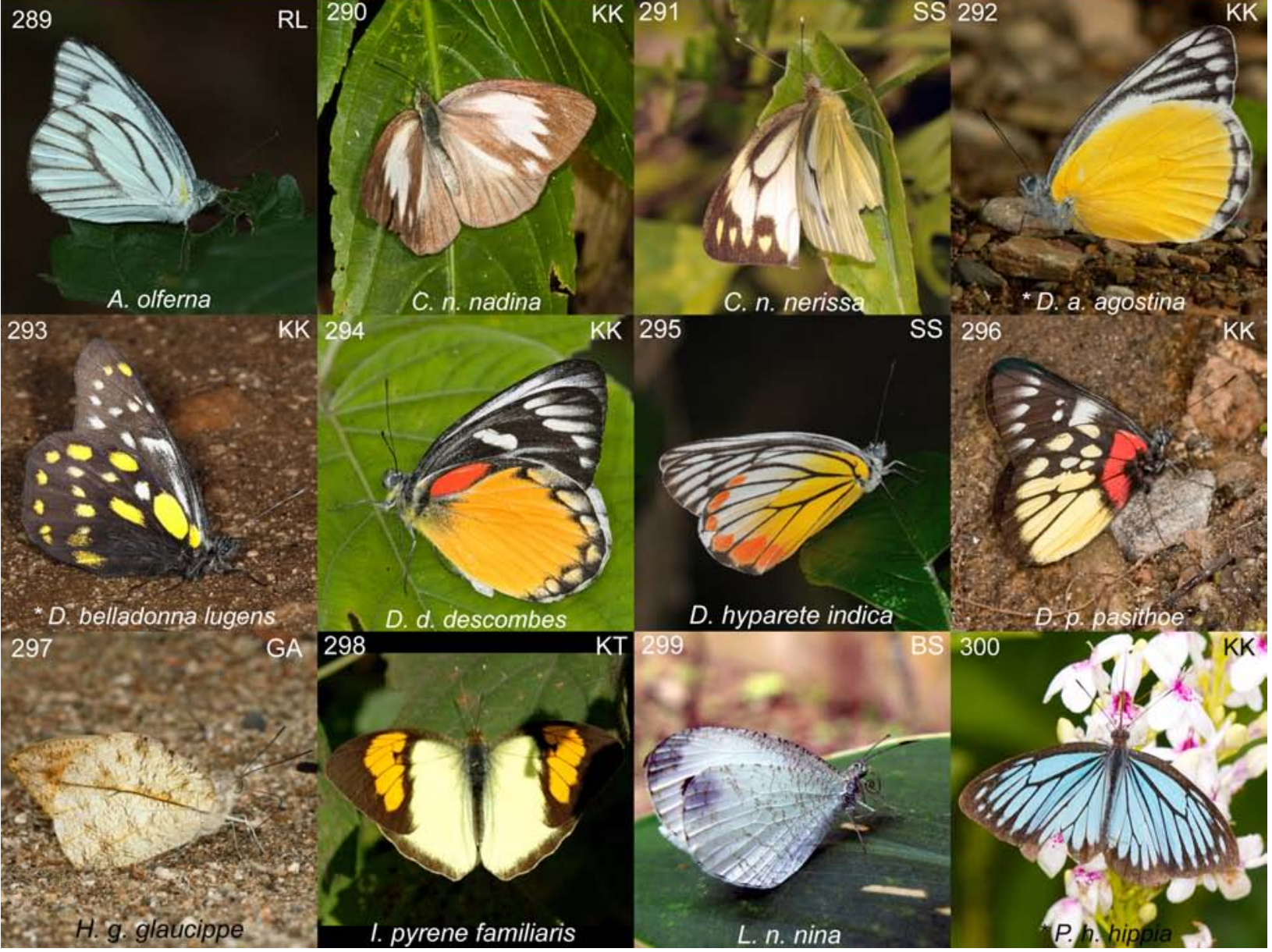
Image 18. Butterflies of the Garo Hills
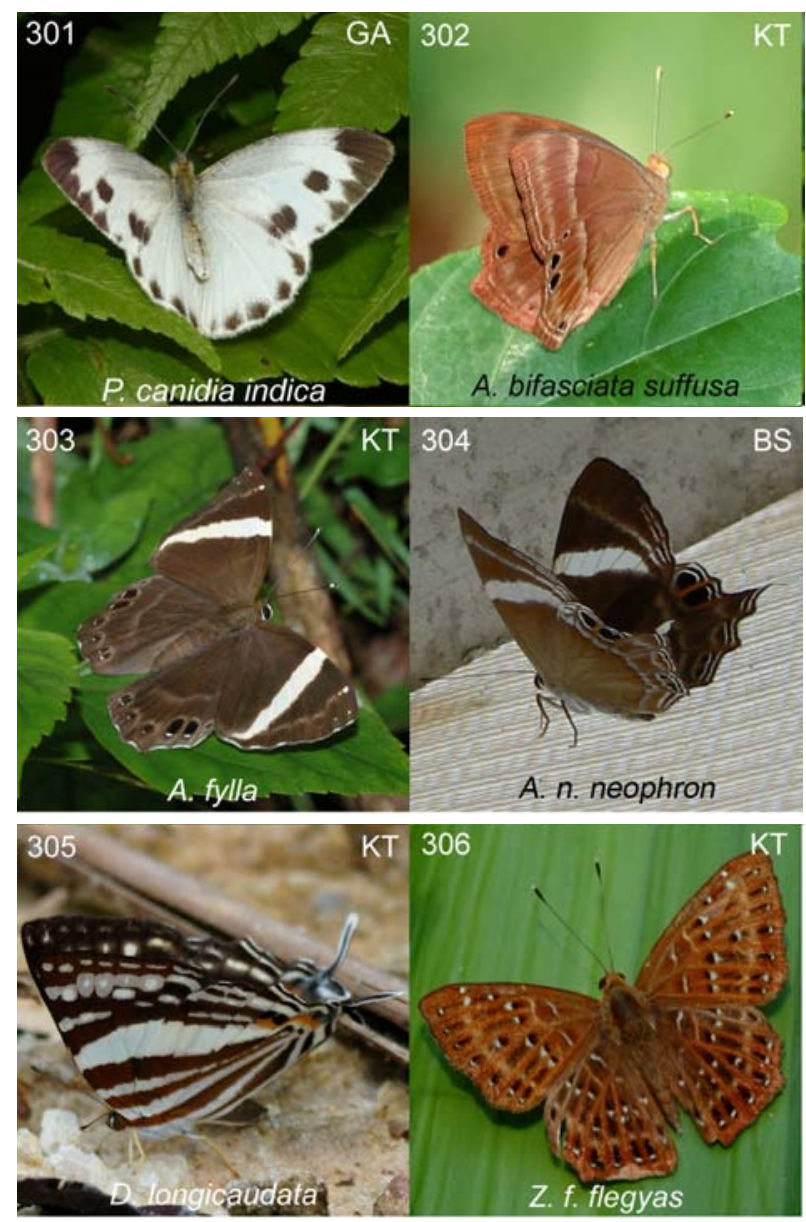

may be occasionally met with mud-puddling along shaded streams in evergreen and semi-evergreen forests in the Garo Hills. It has previously been reported from the Khasi Hills (Cantlie 1956).

5. Hasora badra badra Moore, 1857 - Oriental Common Awl: This is based on a single specimen seen at $0500 \mathrm{hr}$, just before dawn, in early May 2010 at Siju WS. It has previously been reported from the Khasi Hills (Cantlie 1956).

6. Hasora taminatus bhavara Fruhstorfer, 1911 - Himalayan White-banded Awl: Our single record was by KT from the Balpakram Plateau in November 2009. It has previously been reported from the Khasi Hills (Cantlie 1956).

\section{Family Hesperiidae, Subfamily Hesperiinae}

7. Aeromachus jhora creta Evans, 1949 - Khasi Jhora Scrub Hopper: Our record was based on two individuals that KK saw in May 2008, perched on small herbs beside an open stream outside Halwa
Atong Village. It has previously been reported from the Khasi Hills (Cantlie 1956).

8. Ancistroides nigrita diocles Moore, 1865 - Bengal Chocolate Demon: A fairly common species that can be seen along forest paths and streams, basking or settling on bird droppings from which it feeds using its remarkably long proboscis. It has previously been reported from the Khasi Hills (Cantlie 1956).

9. Baoris chapmani Evans, 1937 - Small Paintbrush Swift: Our record of Baoris chapmani was based on a single individual that $\mathrm{KK}$ photographed in the Baghmara RF in November 2009, basking on low vegetation along the Karwani stream. "Baoris penicillata" has been previously reported from the Khasi Hills (Cantlie 1956), but at that time two taxa had been lumped under "penicillata": (a) Baoris chapmani Evans, 1937, and (b) Baoris unicolor Moore, 1883 (Kunte in preparation). The exact identity of the Baoris penicillata specimens recorded from the Khasi Hills will need to be checked from museum specimens mentioned by Cantlie. Baoris penicillata Moore, 1881 (sensu stricto) is Endemic to Sri Lanka, but both chapmani and unicolor are supposed to occur in northeastern India and we report both from the Garo Hills here.

10. Baoris farri Moore, 1878 - Complete Paintbrush Swift: Common in the Garo Hills, frequenting openings along streams in mixed semi-evergreen and evergreen forests. We recorded 10 individuals, eight at Gongrot and two in the Baghmara RF, all in November 2009. This species has previously been reported from the Khasi Hills (Cantlie 1956). It is legally protected in India under Schedule IV of the Wildlife (Protection) Act (Anonymous 1997).

11. Baoris unicolor Moore, 1883 - Black Paintbrush Swift: Our record of Baoris unicolor was based on a single individual that KK photographed beside the Karwani stream in mixed semi-evergreen forest in the Baghmara RF in May 2008. See a note under Baoris chapmani about the taxonomy and previous records of Baoris from the Khasi Hills.

12. Caltoris sp. - Swift: Our record was based on a single specimen that KT photographed in November 2009 on the Balpakram Plateau. Species identifications in this group are based on subtle differences in spotting patterns on the wings and in male genitalia, which could not be investigated closely in our specimen.

13. Cupitha purreea Moore, 1877 - Wax Dart: 
Uncommon in the Garo Hills, occurs along forest paths and streams. Our records are based on only two individuals, one each in May 2008 (Simsang trail, Baghmara RF) and November 2009 (Taidang stream, near Gongrot). It has previously been reported from the Khasi Hills (Cantlie 1956).

14. Halpe spp. - Ace spp.: We have seen four individuals during both seasons at Gongrot, Baghmara RF and Siju WS. Several species of Halpe have been reported from the Khasi Hills (Cantlie 1956), and most of them are expected in the Garo Hills. However, species-level identification of Halpe is challenging. So far, we have not dissected male genitalia to determine the species.

15. Halpe zema zema Hewitson, 1877 - Sikkim Zema Banded Ace: This species cannot be distinguished from Halpe zola Evans, 1937 without dissecting male genitalia, something that we did not do. However, only Halpe zema has been reported from the Khasi Hills before (Cantlie 1956), so we are provisionally assigning the seven individuals that we saw mud-puddling in evergreen forest streams in the Baghmara-Gongrot-Siju landscape to this species. We hope to dissect a few males in the future to confirm this assignment.

16. Hyarotis adrastus praba Moore, 1865 - Bengal Tree Flitter: A single sighting at a forest stream in Panda in December 2009. This subspecies is legally protected in India under Schedule IV of the Wildlife (Protection) Act (Anonymous 1997).

17. Iambrix salsala salsala Moore, 1865 - Eastern Chestnut Bob: very common in the Garo Hills in open forests, along forest edges and around human habitations. Our record is based on 19 individuals recorded from both seasons, although most of the records were from Gongrot in November 2009. It has been reported from the Khasi Hills before (Cantlie 1956).

18. Iton semamora semamora Moore, 1865 - Bengal Common Wight: Our record was based on a single specimen photographed by KT on the Baghmara Plateau in November 2009. This species has apparently not been reported before from the Khasi-Garo Hills complex, so ours is the westernmost record of the species in Meghalaya.

19. Koruthaialos butleri de Nicéville, 1883 - Dark Velvet Bob: Our record was based on a single specimen that KK photographed in mixed deciduous forest in the Baghmara RF in May 2008. This species has been reported from the Khasi Hills before (Cantlie 1956), but it is not common.

20. Matapa aria Moore, 1865 - Common Branded Redeye: Most of our records were of specimens frequenting secondary growth in disturbed evergreen forest patches around Gongrot and nearby villages, from November 2009. This species is widely distributed in the Oriental Region and can sometimes be common in mixed deciduous forests and edges of evergreen forests in northeastern India. The species has been reported from the Khasi Hills before (Cantlie 1956).

21. Matapa cresta Evans, 1949 - Fringed Branded Redeye: We recorded this species from two specimens at Jidung stream and one at Me Cheng Chirang at Gongrot, and one at Siju WS, all in November 2009. It has been reported from the Khasi Hills before (Cantlie 1956).

22. Matapa sasivarna Moore, 1865 - Black-veined Branded Redeye: KT photographed a single specimen on Balpakram plateau in November 2009. The species has been reported from the Khasi Hills before (Cantlie 1956), but does not appear to be common.

23. Notocrypta curvifascia curvifascia Felder \& Felder, 1862 - Chinese Restricted Demon: We recorded several specimens in evergreen forest undergrowth at Gongrot in November 2009. It is common in the Khasi Hills (Cantlie 1956).

24. Notocrypta feisthamelii alysos Moore, 1865 - Himalayan Spotted Demon: Less common than the previous species, although they seem to share the same habitats and many of their habits. It is not rare in the Khasi Hills (Cantlie 1956).

25. Notocrypta paralysos asawa Fruhstorfer, 1911 - Indo-Chinese Common Banded Demon: SS recorded two individuals in Baghmara RF in December 2009. It has been reported to be not rare in the Khasi Hills (Cantlie 1956).

26. Oriens gola pseudolus Mabille, 1883 - Oriental Common Dartlet: Our record is based on a single specimen that KK photographed at Rongrok Stream, Gongrot, in November 2009. It has been reported from the Khasi Hills before (Cantlie 1956).

27. Oriens goloides Moore, 1881 - Smaller Dartlet: Not uncommon in the Garo Hills. Both species of Oriens commonly feed from flowers of small herbs and shrubs, and flit about at forest edges, on paths and 
in treefall gaps in evergreen and riparian forests. This species has been reported from the Khasi Hills before (Cantlie 1956).

28. Parnara sp. - Swift: Three Parnara have been reported from India: (1) Parnara ganga Evans, 1937, (2) Parnara guttatus mangala Moore, 1865, and (3) Parnara bada Moore, 1878. All of them have been reported from the Khasi Hills (Cantlie 1956) and are expected in the Garo Hills. However, species in this group can be determined only by dissecting male genitalia. We have not dissected genitalia from any of the specimens that we have encountered so far, but we hope to do so in the future. Of our 118 sightings, 101 were in May 2008 in agricultural fields around Samrakshan Trust's field office at Baghmara, 15 from jhum areas around Gongrot, and only two were from November 2009 at Gongrot.

29. Pelopidas agna agna Moore, 1865 - Bengal Obscure Branded Swift: This is based on a single specimen that KT photographed on Baghmara Plateau in March 2009. The species has been reported from the Khasi Hills before (Cantlie 1956).

30. Pelopidas assamensis de Nicéville, 1882 - Great Swift: Not uncommon in fallow jhum fields overgrown with shrubs, as well as in small openings inside dense forests. It has been reported from the Khasi Hills before (Cantlie 1956). This species is legally protected in India under Schedule IV of the Wildlife (Protection) Act (Anonymous 1997).

31. Pirdana major Evans, 1932 - Himalayan Green-striped Palmer: Our record from the Garo Hills was based on a single specimen seen by KK at Taidang stream near Gongrot in November 2009. It was a fresh female seen on a clouded afternoon, in one of the most shaded parts of an evergreen forest stream. It was perched at two meters above ground on a branch overhanging the stream, and was not active. The picture included in image 4 may be the first picture of a live specimen ever taken. This is a very rare species and, as far as we know, it has not been reported in literature from the Garo-Khasi Hills complex before. The Natural History Museum, London, has only one male taken in Sikkim, and eight males and two females taken in "Assam" (Evans 1949), some of which may have been taken in the Khasi Hills. This species is sometimes included as a subspecies of Pirdana hyela Hewitson 1867.

32. Pithauria stramineipennis stramineipennis
Wood-Mason \& de Nicéville, 1886 - Assam Light Straw Ace: Our records are based on two males mudpuddling at Siju WS and a third specimen at Karwani in May 2010. The species has been reported from the Khasi Hills before (Cantlie 1956).

33. Polytremis lubricans lubricans HerrichSchäffer, 1869 - Oriental Contiguous Swift: This species seems to be uncommon in the Garo Hills. Our record was based on a single sighting from Gongrot in May 2009. It has been reported from the Khasi Hills before (Cantlie 1956), and is frequently met with in open areas and secondary vegetation in northeastern India. This subspecies is legally protected in India under Schedule IV of the Wildlife (Protection) Act (Anonymous 1997).

34. Potanthus spp. - Dart spp.: Eleven Potanthus species have been reported from the Khasi Hills before: (1) rectifasciata Elwes \& Edwards, 1897 - Branded Dart, (2) trachala tytleri Evans, 1914 - Manipur Broad Bident Dart, (3) pallida Evans, 1932 - Pallid Dart, (4) pseudomaesa clio Evans, 1932 - Himalayan Common Dart, (5) juno Evans, 1932 - Myanmarese Dart, (6) sita Evans, 1932 - Sita Dart, (7) confucius dushta Fruhstorfer, 1911 - Indo-Chinese Dart, (8) lydia Evans, 1934 - Assam Dart, (9) ganda Fruhstorfer 1911 - Sumatran Dart, (10) nesta Evans, 1934 - Nesta Dart, and (11) palnia Evans, 1914 - Palni Dart (Cantlie 1956). Most of them are expected to be found in the Garo Hills. We have come across only two Potanthus in the Garo Hills so far and did not dissect their genitalia, so we do not know their specific identities.

35. Psolos fuligo subfasciatus Moore, 1878 - Indian Dusky Partwing: Uncommon but widespread in NE India and elsewhere in Indo-China, which we recorded based on two specimens seen by KT from the Balpakram Plateau and Nokrek NP.

36. Scobura isota Swinhoe, 1893 - Khasi Forest Bob: We saw two specimens feeding from a bird dropping inside evergreen forest near Gongrot, and one inside Baghmara RF. The species is not uncommon in northeastern India, and has been reported from the Khasi Hills before (Cantlie 1956).

37. Sebastonyma dolopia Hewitson, 1868 - Tufted Ace: Our three sightings were from Siju WS in May 2010. This is a rather uncommon species that has been reported from the Khasi Hills before (Cantlie 1956).

38. Spialia galba Fabricius, 1793 - Indian Grizzled Skipper: KT recorded a single specimen on 
the Balpakram Plateau in November 2009 and SS recorded another specimen near Halwa Atong in May 2010. The species is locally frequent in the Khasi Hills (Cantlie 1956).

39. Suada swerga swerga de Nicéville, 1883 - Indian Grass Bob: This was common in the Garo Hills in November 2009. KK recorded five fresh specimens, two of them mating, the rest basking among or near bamboo clumps along streams in semievergreen forests.

40. Telicota bambusae bambusae Moore, 1878 - Oriental Dark Palm-Dart: We usually saw males of this species mud-puddling or feeding from bird droppings in sunny patches along streams in evergreen forests.

41. Zographetus ogygia ogygia Hewitson, 1866 - Sumatran Purple-spotted Flitter: Our record was based on a single specimen that KK photographed in the Matcha Nokpante Community Reserve outside Baghmara in November 2009. The species has apparently not been reported from the Garo-Khasi Hills complex before (Cantlie 1956).

42. Zographetus satwa de Nicéville, 1884 - Purple and Gold Flitter: Our record was based on a single sighting from scrub forest near Botra village in Baghmara RF in December 2009. The species has been reported from the Khasi Hills before (Cantlie 1956), and is not uncommon in northeastern India.

\section{Family Hesperiidae, Subfamily Pyrginae}

43. Celaenorrhinus asmara consertus de Nicéville, 1890 - Khasi White-banded Flat: GA, RL and SS photographed a single specimen in Baghmara RF in May 2010. It has been reported from the Khasi Hills before (Cantlie 1956), but it is an uncommon species in northeastern India.

44. Gerosis bhagava bhagava Moore, 1865 - Bengal Yellow-breasted Flat: In Nov. 2009, KK saw two males early in the morning on a sparsely vegetated ridge in the Matcha Nokpante Community Reserve outside the Baghmara Town. The pair was engaged in a fierce contest over closely placed vantage points, which were approximately $3 \mathrm{~m}$ apart and $1 \mathrm{~m}$ above the ground on small shrubs. The pair would take off every few minutes, chasing each other at amazingly high speeds, minutes on end, going on for over half an hour. The contestants gave up and departed only when sunlight began to brighten up the surroundings.
The species has apparently not been reported from the Khasi Hills before (Cantlie 1956), although it is not uncommon in northeastern India.

45. Gerosis phisara phisara Moore, 1884 - Khasi Dusky Yellow-breast Flat: This species is common in the Garo Hills in evergreen forests, where several individuals were seen mud-puddling. It is also common in the Khasi Hills (Cantlie 1956).

46. Gerosis sinica narada Moore, 1884 - Sikkim White Yellow-breasted Flat: Our Garo Hills record is based on a single specimen seen mud-puddling at Taidang stream near Gongrot. It is common in the Khasi Hills (Cantlie 1956).

47. Odontoptilum angulata angulata Felder, 1862 - Oriental Chestnut Angle: The species was very common in the Garo Hills during the pre-monsoon season when we saw 23 specimens, but none during our November-December 2009 visits. Males were seen mud-puddling or chasing other butterflies from near their vantage points. The species has been reported from the Khasi Hills before (Cantlie 1956).

48. Pseudocoladenia dan fabia Evans, 1949 - Himalayan Fulvous Pied Flat: We found this to be common in both seasons in disturbed evergreen forests. It has been reported from the Khasi Hills before (Cantlie 1956).

49. Sarangesa dasahara dasahara Moore, 1865 - Bengal Common Small Flat: This species frequents forest paths, stream-sides and forest edges in the Garo Hills. It is common in the Khasi Hills (Cantlie 1956)

50. Tagiades gana athos Plötz, 1884 - Bengal Suffused Snow Flat: It is common inside evergreen forests in the Garo Hills, typically along forest paths and streams. It is also reportedly common in the Khasi Hills (Cantlie 1956).

51. Tagiades japetus ravi Moore, 1865 - Himalayan Common Snow Flat: This species is common in the Garo Hills, and shares its habitat and habits with the previous species. It is also common in the Khasi Hills (Cantlie 1956).

52. Tagiades litigiosa litigiosa Möschler, 1878 - Sylhet Water Snow Flat: We had a single record of this species from Balpakram Plateau in November 2009. It is common in the Khasi Hills (Cantlie 1956). 
Family Lycaenidae (Blues, Hairstreaks, Coppers, etc.)

\section{Subfamily Curetinae}

53. Curetis bulis bulis Westwood, 1851: Himalayan Bright Sunbeam: We have so far seen only one specimen of this species near Gongrot in May 2008. It is common in the Khasi Hills (Cantlie 1952), and elsewhere in northeastern India.

54. Curetis dentata dentata Moore, 1879 - Indian Toothed Sunbeam: Our Garo Hills record is based on six specimens that we photographed mud-puddling near Gongrot. The species is "not rare" in the Khasi Hills (Cantlie 1952).

\section{Family Lycaenidae, Subfamily Lycaeninae}

55. Heliophorus epicles latilimbata Eliot, 1963 - Sikkim Purple Sapphire: This species is common in the Garo Hills on forest paths and at forest edges. Both sexes were frequently encountered basking with their wings partially spread. It is also common in the Khasi Hills (Cantlie 1952), in the eastern Himalaya and elsewhere in northeastern India.

56. Heliophorus indicus Fruhstorfer, 1908 - Indian Sapphire: Our Garo Hills record is based on a single male that KT photographed at Nokrek NP in Nov. 2009. The species is distributed widely but is not common anywhere. This is in contrast with the Khasi Hills records, where it is reportedly common (Cantlie 1952). We suspect that this must be true for Heliophorus epicles latilimbata, not Heliophorus indicus (Cantlie listed Heliophorus epicles indicus as common, but did not mention the commoner Heliophorus epicles latilimbata).

\section{Family Lycaenidae, Subfamily Miletinae}

57. Allotinus drumila drumila Moore, 1865 - Himalayan Crenulate Mottle: Our sole record for the Garo Hills was from May 2010 near Gongrot. This species is cryptically patterned on the underside and has a habit of perching motionless for hours in exposed spots, and is therefore easily overlooked. Several specimens have been collected in the past from around Cherrapunjee in the Khasi Hills (Cantlie 1952). This species is legally protected in India under Schedule I of the Wildlife (Protection) Act (Anonymous 1997).

58. Miletus chinensis assamensis Doherty, 1891 - Assam Common Mottle: Two specimens of this widespread species were photographed in the
Baghmara RF in May 2010.

59. Spalgis epeus epeus Westwood, 1851 - Oriental Apefly: This tiny species is remarkable for being carnivorous in larval stages, feeding on mealy bugs rather than plants. Its wing coloration is dull and the adults are easily overlooked. We recorded two specimens flying together at Karwani stream in Baghmara RF in May 2010. It was reported to be "not rare but scarce" on the southern side of the Khasi Hills on border with Sylhet (Cantlie 1952).

\section{Family Lycaenidae, Subfamily Polyommatinae}

60. Acytolepis puspa gisca Fruhstorfer, 1910 - Himalayan Common Hedge Blue: We have recorded several individuals in the Garo Hills, usually mudpuddling along evergreen forest streams. This is a widely distributed and very common species in India and elsewhere in the Oriental Region, including in the Khasi Hills (Cantlie 1952).

61. Anthene emolus emolus Godart, 1823 - Bengal Common Ciliate Blue: This is abundant in the Garo Hills, where males were commonly seen either mudpuddling or basking along stream-sides, forest paths and around jhum areas. It is also common in the Khasi Hills (Cantlie 1952).

62. Anthene lycaenina lycambes Hewitson, 1878 - Shan Pointed Ciliate Blue: We have recorded four specimens during our surveys so far. The species is somewhat similar to Anthene emolus emolus, with which it shares many habits and its habitat. However, it is much less common compared to A.e. emolus, and is also reportedly uncommon in the Khasi Hills (Cantlie 1952). This species is legally protected in India under Schedule II of the Wildlife (Protection) Act (Anonymous 1997).

63. Caleta elna noliteia Fruhstorfer, 1918 - IndoChinese Elbowed Pierrot: This species is fairly common in the Garo Hills, and most of our records were of mud-puddling males. It is frequently seen in the Khasi Hills (Cantlie 1952).

64. Castalius rosimon rosimon Fabricius, 1775 - Continental Common Pierrot: This species was common around human habitations and in secondary, fairly open forests. It is common in the Khasi Hills (Cantlie 1952) and elsewhere in the Oriental Region.

65. Catochrysops panormus exiguus Distant, 1886 - Malay Silver Forget-me-not: This was uncommon in secondary forests, where males were sometimes 
encountered mud-puddling. It is common in the Khasi Hills (Cantlie 1952).

66. Catochrysops strabo strabo Fabricius, 1793 - Oriental Forget-me-not: This species was uncommon in deciduous forest patches and in secondary evergreen forests, and usually encountered mud-puddling. It is common in the Khasi Hills (Cantlie 1952).

67. Celastrina lavendularis limbata Moore, 1879 - Eastern Plain Hedge Blue: It was found to be common in the Garo Hills in both the seasons, and especially frequently encountered along evergreen forest streams near Gongrot, where males were usually seen mudpuddling. It has been reported from the Khasi Hills (Cantlie 1952).

68. Chilades lajus lajus Stoll, 1780 - Indian Lime Blue: It was common around human habitations in the Garo Hills. Several individuals were seen courting and laying eggs around the Samrakshan Trust office in Baghmara. It is also common in the Khasi Hills (Cantlie 1952).

69. Discolampa ethion ethion Westwood, 1851 - Oriental Banded Blue Pierrot: BS recorded a single specimen of this species at Taidang stream near Gongrot. This was known from upper and southern Assam but apparently had not been reported from the Khasi Hills proper (Cantlie 1952).

70. Euchrysops cnejus cnejus Fabricius, 1798 - Oriental Gram Blue: This species occurs in open, drier habitats, and our only two records were from around human habitations or otherwise degraded habitats. It is also uncommon in the Khasi Hills (Cantlie 1952). This species is legally protected in India under Schedule II of the Wildlife (Protection) Act (Anonymous 1997).

71. Jamides alecto eurysaces Fruhstorfer, 1915 - Himalayan Metallic Cerulean: This was common in the Garo Hills, especially during the pre-monsoon season in the evergreen forests around Gongrot. It is apparently not common in the Khasi Hills (Cantlie 1952).

72. Jamides bochus bochus Stoll, 1782 - Indian Dark Cerulean: We usually recorded this species in open areas at forest edges. It is common in the Khasi Hills (Cantlie 1952), as elsewhere in India and the Oriental Region.

73. Jamides celeno celeno Cramer, 1775 - Oriental Common Cerulean: This species was very common around human habitations, along forest streams and at forest edges. The dry and wet season forms are strikingly different, and each was common in the respective season in the Garo Hills. Males were often found mud-puddling, and both sexes were seen feeding from flowers of small shrubs. It is apparently not very common in the Khasi Hills (Cantlie 1952).

74. Jamides elpis pseudelpis Butler, 1879 - False Glistening Cerulean: Our only record for the Garo Hills was a single individual from Siju WS. It is "not rare" in the Khasi Hills (Cantlie 1952).

75. Jamides pura pura Moore, 1886 - Continental White Cerulean: This species cannot be distinguished from Jamides celeno without viewing the upperside of the forewings. In $J$. celeno the black border increases in width at the forewing tip and may be fairly broad, whereas it is threadlike and uniformly narrow in $J$. pura. In the Garo Hills, J. pura tended to occur in slightly moister habitats but otherwise it was identical to $J$. celeno in its habits. The species is generally uncommon in northeastern India, but KK encountered it frequently around Gongrot in November 2009. It is apparently not uncommon even in the Khasi Hills (Cantlie 1952).

76. Lampides boeticus Linnaeus, 1767 - Pea Blue: This species usually appears sporadically in all types of open habitats from human habitations to bare hilltops, and our Garo Hills record was based on a single sighting from the Nokrek NP. It is reportedly common in the Khasi Hills (Cantlie 1952).

77. Lestranicus transpectus Moore, 1879 - Whitebanded Hedge Blue: We have so far seen only a single individual of this uncommon but widely distributed species. It should turn up in more numbers, especially in Nokrek NP and elsewhere at mid-elevations. It has apparently not been recorded in the Khasi Hills before (Cantlie 1952).

78. Megisba malaya sikkima Moore, 1884 - Variable Malayan: We found this species to be very common along forest paths, edges and streams. Males were often seen mud-puddling and both sexes were seen feeding from flowers. It is also common in the Khasi Hills (Cantlie 1952).

79. Nacaduba beroe gythion Fruhstorfer, 1916 - Assam Opaque Six-Lineblue: This species is common in the Garo Hills, where males were frequently seen mud-puddling. It is also common in the Khasi Hills (Cantlie 1952).

80. Nacaduba hermus nabo Fruhstorfer, 1916 
- Assam Pale Four-Lineblue: This and the next species were much less common compared to N. beroe. All our sightings were of mud-puddling males. It is also rare in the Khasi Hills (Cantlie 1952).

81. Nacaduba kurava euplea Fruhstorfer, 1916 - Sikkim Transparent Six-Lineblue: This was not very common in the Garo Hills, and most of our records were of mud-puddling males. It is reportedly common in the Khasi Hills (Cantlie 1952).

82. Neopithecops zalmora zalmora Butler, 1870 - Myanmar Common Quaker: This species was common in May 2010 along forest streams, where males were usually seen mud-puddling, although there were also two records from December 2009. It is common in the Khasi Hills (Cantlie 1952).

83. Prosotas aluta coelestis Wood-Mason \& de Nicéville, 1886 - Assam Banded Lineblue: This species was fairly common in the Baghmara RF and near Gongrot, perhaps due to the lower elevation in the Garo Hills, which it seems to prefer. However, it is reportedly uncommon to rare in the Khasi Hills (Cantlie 1952). This subspecies is legally protected in India under Schedule II of the Wildlife (Protection) Act (Anonymous 1997).

84. Prosotas bhutea de Nicéville, 1883 - Bhutia Lineblue: This is an uncommon species, recorded from two males seen along Taidang stream near Gongrot. In appearance and habits this species was very similar to Prosotas nora.

85. Prosotas dubiosa indica Evans, 1925 - Indian Tailless Lineblue: This was very common in the Garo Hills, where we usually recorded males mud-puddling. It is also common in the Khasi Hills (Cantlie 1952).

86. Prosotas lutea sivoka Evans, 1910 - Teesta Brown Lineblue: This was common in the Garo Hills, especially during the post-monsoon when males were usually seen mud-puddling. It has also been reported from the Khasi Hills (Cantlie 1952).

87. Prosotas nora ardates Moore, 1874 - Indian Common Lineblue: This was common in the Garo Hills, especially during the post-monsoon. Males were sometimes seen mud-puddling, congregated in small groups. It is also very common in the Khasi Hills (Cantlie 1952).

88. Pseudozizeeria maha maha Kollar, 1844 - Himalayan Pale Grass Blue: A small but conspicuous butterfly that was usually encountered around human habitations, flying low over herbs and small shrubs. It is also common in the Khasi Hills (Cantlie 1952).

89. Talicada nyseus khasiana Swinhoe, 1893 - Khasi Red Pierrot: We only saw two individuals, although this is a highly localized species, which may sometimes be locally common. It is reportedly uncommon around Cherrapunjee and not recorded elsewhere in the Khasi Hills (Cantlie 1952), although it should be present throughout the Garo-Khasi Hills complex.

90. Tarucus ananda de Nicéville, 1883 - Dark Pierrot: Our only two Garo Hills records were of mudpuddling males in evergreen forests near Gongrot and in the Baghmara RF. This species is apparently common in the Khasi Hills (Cantlie 1952). It is legally protected in India under Schedule IV of the Wildlife (Protection) Act (Anonymous 1997).

91. Tarucus venosus Moore, 1882 - Veined Pierrot: Unlike the previous species, this is an inhabitant of slightly more open habitats. Our only record was by KT from the Balpakram Plateau.

92. Udara dilecta dilecta Moore, 1879 - Himalayan Pale Hedge Blue: Our only record was from Baghmara $\mathrm{RF}$, although this species is apparently common in the Khasi Hills (Cantlie 1952).

93. Zizeeria karsandra Moore, 1865 - Dark Grass Blue: We have recorded only one individual around Baghmara in May 2010, and we are surprised that we did not find this species to be common in the Garo Hills. It is widely distributed and common everywhere it occurs, usually in open areas and often around human habitations and cultivated fields. It seems that we have not sampled its habitat properly so far. However, this species is apparently uncommon even in the Khasi Hills (Cantlie 1952).

94. Zizina otis otis Fabricius, 1787 - Oriental Lesser Grass Blue: This species occurs in the same habitats as the previous species. Our two records were from the neighborhood of Halwa Atong. It is common in the Khasi Hills (Cantlie 1952).

95. Zizula hylax hylax Fabricius, 1775 - Indian Tiny Grass Blue: We recorded a single individual in dry scrub habitat near the Botra Village in December 2009. The species is common in the Khasi Hills (Cantlie 1952).

\section{Family Lycaenidae, Subfamily Theclinae}

96. Amblypodia anita dina Fruhstorfer, 1907 - Indian Purple Leaf Blue: Our single record was from 
Rani Miksuram Stream near Halwa Atong Village, by BS.

97. Ancema blanka minturna Fruhstorfer, 1912 - Blue-streaked Silver Royal: We came across three males of this species feeding from dead crabs (including on the ones set by us as baits) in Jidung Stream near Gongrot in November 2009. This subspecies is legally protected in India under Schedule II of the Wildlife (Protection) Act (Anonymous 1997).

98. Ancema ctesia ctesia Hewitson, 1865 - Himalayan Bi-spot Royal: SS had a single sighting of this distinctive species from Karwani in December 2009, which needs further confirmation since SS was unable to photograph the specimen. This species is rare in the Khasi Hills (Cantlie 1952) and elsewhere in northeastern India.

99. Arhopala abseus indicus Riley, 1923 - Indian Aberrant Oakblue: Our single record was based on an individual that was among the congregation of over a hundred Arhopala that KK and RL saw in deciduous forest along the Simsang trail in the Baghmara RF in May 2008 (see details under Arhopala centaurus pirithous). This species is rare throughout its range, but has been reported from the Khasi Hills before (Cantlie 1952).

100. Arhopala ammonides elira Corbet, 1941 - Assam Little Cerulean Oakblue: Our only record was from the evergreen forest at Karwani, Baghmara RF, on 30 April 2010. Not much is known about this species from India, so this may be a useful spot record.

101. Arhopala atrax Hewitson, 1862 - Indian Oakblue: Our two records were from Simsang trail in December 2009 and Karwani stream in May 2010, both in Baghmara RF.

102. Arhopala centaurus pirithous Moore, 1883 - Bengal Centaur Oakblue: KK and RL witnessed a remarkable congregation of Arhopala from 1100 to $1230 \mathrm{hr}$ on 2 May 2008 in a degraded deciduous forest patch along the Simsang trail in the Baghmara RF. The congregation was composed of over a hundred Arhopala centaurus pirithous, only 14 Arhopala fulla ignara, and a single Arhopala abseus indicus, all intermingled. All the specimens from this congregation that we captured for close inspection turned out to be males, except a female of Arhopala fulla ignara. The butterflies had congregated in a relatively dense patch of large shrubs and trees, no larger than approximately 20x20 m, which was more shaded than the surrounding deciduous forest. However, it was not particularly hot or dry that day, so we have no clue why butterflies had congregated there, especially when all the other butterfly species observed around that time in that area were actively mud-puddling, basking or engaged in other usual activities. Some males from the Arhopala congregation did bask with wings $3 / 4^{\text {th }}$ spread, but none were feeding. Most were resting on the upperor undersides of leaves, both in the open and in shade of the dense vegetation. They were wary, however, and difficult to catch. We hardly saw any Arhopala during the post-monsoon, so pre-monsoon seems to be the peak flight period for most Arhopala in this area. This species is reportedly "not rare" in the Khasi Hills (Cantlie 1952).

103. Arhopala fulla ignara Riley \& Godfrey, 1921 - Thai Spotless Oakblue: See above under Arhopala centaurus pirithous for majority of our sightings of this species. This subspecies is legally protected in India under Schedule II of the Wildlife (Protection) Act (Anonymous 1997).

104. Arhopala perimuta perimuta Moore, 1857 Sylhet Yellowdisc Tailless Oakblue: We sighted six individuals at Panda, Gongrot and Balpakram Plateau in November and December 2009, with no premonsoon sightings, implying a post-monsoon flight period. The species has previously been reported as "not rare" in the Khasi Hills (Cantlie 1952).

105. Bindahara phocides phocides Fabricius, 1793 - Thai Plane: Our two records were from Karwani in December 2009 and Bhawanipur in May 2010, both in Baghmara RF. The species is rare in northeastern India in general, and apparently known only from four specimens in the Khasi Hills (Cantlie 1952). This species is legally protected in India under Schedule II of the Wildlife (Protection) Act (Anonymous 1997).

106. Cheritra freja evansi Cowan, 1965 - Khasi Common Imperial: We have a few records from evergreen forests from Gongrot and Baghmara RF. It is reportedly common in the Khasi Hills (Cantlie 1952).

107. Chliaria othona othona Hewitson, 1865 - Oriental Orchid Tit: Our four records were of males mud-puddling along evergreen forest streams at Gongrot, Karwani Stream and Siju WS. It is "not rare but scarce" in the Khasi Hills (Cantlie 1952). This species is legally protected in India under Schedule I of the Wildlife (Protection) Act (Anonymous 1997). 
108. Creon cleobis cleobis Godart, 1824 - Bengal Broad-tail Royal: SS recorded a single individual by the roadside near Dabit Chirring in May 2010. The species is not rare in the Khasi Hills (Cantlie 1952).

109. Dacalana penicilligera de Nicéville, 1890 - Double-tufted Royal: SS photographed a single individual along the Karwani stream in May 2010.

110. Flos apidanus ahamus Doherty, 1891 - Assam Plain Plushblue: This record is based on just the right hindwing that $\mathrm{KK}$ found in a dry streambed at Siju WLS on 22 November 2009. This subspecies is legally protected in India under Schedule II of the Wildlife (Protection) Act (Anonymous 1997).

111. Horaga onyx onyx Moore, 1857 - Variable Common Onyx: We have so far seen two individuals in the Garo Hills, one just outside the Baghmara town in an orchard in May 2008, and one in November 2009 at Gongrot. It is rare and known apparently only from three specimens in the Khasi Hills (Cantlie 1952). This species is legally protected in India under Schedule II of the Wildlife (Protection) Act (Anonymous 1997).

112. Hypolycaena erylus himavantus Fruhstorfer, 1912 - Sikkim Common Tit: This was one of the most abundant lycaenids in our sampling. This was seen in both seasons but evidently much more abundant during the post-monsoon, especially near Gongrot and at Karwani Stream. This is also very common in the Khasi Hills (Cantlie 1952).

113. Loxura atymnus continentalis Fruhstorfer, 1912 - Continental Yamfly: We saw this commonly basking along forest paths and edges. It is "not rare" in the Khasi Hills (Cantlie 1952).

114. Lycaenid sp.: SS managed to take just one picture of this Thecline at Karwani Stream in December 2009 but we are clueless about what this species may be, or even to which genus it may belong. We have been unable to find a match in any of the books that we have referred to (Wynter-Blyth 1957; Pinratana 1981; d'Abrera 1986; Corbet et al. 1992; Io 2000), or to any of the specimens available in the Museum of Comparative Zoology at Harvard University. Please contact us if you have any comments or suggestions.

115. Rapala dieneces dieneces Hewitson, 1878 - Malay Scarlet Flash: We recorded one individual each at Me Cheng Chirang near Halwa Atong and Balpakram Plateau, both at forest edges. The species is rare in the Khasi Hills (Cantlie 1952).

116. Rapala manea schistacea Moore, 1879
- Bengal Slate Flash: We saw three individuals during the pre-monsoon at Baghmara RF and near Gongrot. It is "not rare" in the Khasi Hills (Cantlie 1952).

117. Rapala pheretima petosiris Hewitson, 1863 - Indian Copper Flash: This was fairly common in the Garo Hills, where several individuals were seen feeding from flowers of a shrub at the edge of the forest in Baghmara RF. It is "not rare" in the Khasi Hills (Cantlie 1952).

118. Rapala varuna orseis Hewitson, 1863 - Sumatran Indigo Flash: We recorded two individuals at Siju WS and Panda Chirring in December 2009. It is "not rare but scarce" in the Khasi Hills (Cantlie 1952). This subspecies is legally protected in India under Schedule II of the Wildlife (Protection) Act (Anonymous 1997).

119. Remelana jangala ravata Moore, 1865 - Northern Chocolate Royal: Our three records were of males mud-puddling in evergreen forest streams. The species has been reported from the Khasi Hills (Cantlie 1952).

120. Spindasis lohita himalayanus Moore, 1884 - Himalayan Long-banded Silverline: We saw three males mud-puddling along evergreen forest streams at Gongrot, and a female basking at the edge of the forest in Siju WS. It is "not rare" in the Khasi Hills (Cantlie 1952). This subspecies is legally protected in India under Schedule II of the Wildlife (Protection) Act (Anonymous 1997).

121. Spindasis syama peguanus Moore, 1884 - Pegu Club Silverline: We found it commonly in open spaces at forest edges and in open, dry streambeds, especially around Gongrot. It is "not rare but scarce" in the Khasi Hills (Cantlie 1952).

122. Surendra quercetorum quercetorum Moore, 1857 - Himalayan Common Acacia Blue: In our sampling in the Garo Hills, females were much more common than males, which also seems to be true of other populations of this species elsewhere in India. They were mostly seen in open forests and at forest edges or along forest paths where their larval host plants, straggling Acacia, were growing. The species is common in the Khasi Hills (Cantlie 1952).

123. Ticherra acte acte Moore, 1857 - Himalayan Blue Imperial: Our record of this uncommon species was based on a single specimen seen by SS at Taidang stream near Gongrot. It has been reported from the Khasi Hills (Cantlie 1952). 
124. Yasoda tripunctata tripunctata Hewitson, 1863 - Sylhet Branded Yamfly: We recorded two individuals, both during the pre-monsoon in evergreen forests at Gongrot and Karwani stream. This rare species was apparently known from the Khasi Hills only from two specimens (Cantlie 1952). This species is legally protected in India under Schedule II of the Wildlife (Protection) Act (Anonymous 1997).

125. Zeltus amasa amasa Hewitson, 1865 - Indian Fluffy Tit: This was one of the most common Lycaenids in our sampling. Dozens of individuals were seen mud-puddling in evergreen forests along Taidang and Jidung streams near Gongrot. It is reportedly "not rare" in the Khasi Hills (Cantlie 1952).

\section{Family Nymphalidae (Brush-footed Butterflies) Subfamily Apaturinae}

126. Apatura ambica ambica Kollar, 1844 - East Himalayan Purple Emperor: Both our records were of mud-puddling males at Taidang stream near Gongrot. It is common in the Khasi Hills (Parsons \& Cantlie 1948).

127. Euripus nyctelius nyctelius Doubleday, 1845 - Sylhet Courtesan: We saw seven males in both seasons, they were perched on vantage points at the tips of branches of shrubs and small trees, from where they chased other butterflies passing nearby. Females of this species are rarely encountered and we did not see any in the Garo Hills. The species is "not uncommon" in the Khasi Hills (Parsons \& Cantlie 1948).

128. Herona marathus marathus Doubleday, 1848 - Assam Pasha: This is a very rare species. Our only record was from Nokrek NP by KT. It has apparently not been reported from the Khasi Hills before.

129. Rohana parisatis parisatis Westwood, 1850 - Assam Black Prince: We saw three males mudpuddling in evergreen forest streams. It is common in the Khasi Hills (Parsons \& Cantlie 1948).

\section{Family Nymphalidae, Subfamily Biblidinae}

130. Ariadne ariadne pallidior Fruhstorfer, 1899 - Large Angled Castor: This species was common in secondary forest, at forest edges and around human habitations in the Garo Hills, especially during the premonsoon. It is "not rare" in the Khasi Hills (Parsons \& Cantlie 1948).

131. Ariadne merione tapestrina Moore, 1884 - Intricate Common Castor: This species was much less numerous compared to the last species but occurred in the same habitats. It has been reported from the Khasi Hills before (Parsons \& Cantlie 1948).

\section{Family Nymphalidae, Subfamily Charaxinae}

132. Charaxes arja arja Felder \& Felder, 1866 - Bengal Pallid Nawab: This species was recorded in both seasons but was more common during the postmonsoon. It flew in the same places as Charaxes athamas athamas: along forest paths, streams and edges. The two species were often seen on the same mud-puddling spots or on baits of rotting crabs around Gongrot. It is presumably common in the Khasi Hills (Parsons \& Cantlie 1948).

133. Charaxes athamas athamas Drury, 1770 - Oriental Common Nawab: This species was very commonly seen mud-puddling along forest streams at Taidang, Jidung and Rani Miksuram streams near Gongrot. It was also a major draw on baits of rotting crabs, where nearly a dozen individuals could be attracted at any time of the day. The species is also common in the Khasi Hills (Parsons \& Cantlie 1948).

134. Charaxes bernardus hierax Felder \& Felder, 1866 - Variable Tawny Rajah: This species was very common in forested areas of the Garo Hills. Males were sometimes seen mud-puddling, but most of the individuals that we recorded were drawn to baits of rotting crabs that we had set out to attract nymphalids. Males of this species are polymorphic in northeastern India, and the individuals that we caught for closer inspection turned out to belong to the following three forms: hierax, pleistoanax and hipponax. The species is reportedly "not rare" in the Khasi Hills (Parsons \& Cantlie 1948).

135. Charaxes eudamippus eudamippus Doubleday, 1843 - Himalayan Great Nawab: Our Garo Hills record is based on a single specimen that BS photographed feeding on a rotting crab at Taidang Stream near Gongrot in May 2008. This species may be more common than our sampling suggests so far; we have probably missed its peak flight season in this area, which may be March and April. It is apparently common in the Khasi Hills (Cantlie 1952), and has recently been reported further southwest in Similipal National Park (Nair 2011).

136. Charaxes kahruba Moore, 1895 - Variegated Rajah: We have so far recorded four specimens in the Garo Hills, most of them around Gongrot, and all of 
them mud-puddling or feeding on rotting crabs. The species is reportedly very rare in the Khasi Hills, and apparently only two specimens were collected by Parson \& Cantlie (1948).

137. Charaxes marmax marmax Westwood, 1847 - Sylhet Yellow Rajah: KK recorded three individuals at Taidang and Rani Miksuram streams near Gongrot and Halwa Atong in November 2009. This species is generally rare throughout its range, it is "scarce" in the Khasi Hills (Parsons \& Cantlie 1948), and is legally protected in India under Schedule II of the Wildlife (Protection) Act (Anonymous 1997).

138. Charaxes moori sandakanus Fruhstorfer, 1895 - Margined Malayan Nawab: We have two records of this very rare species, one by BS at Anakpatal, Rongai Stream, Hangsapal, in June 2008, and the other by GA and RL at Taidang stream near Gongrot in May 2010. These sightings are important because this subspecies is legally protected in India under Schedule I of the Wildlife (Protection) Act (Anonymous 1997).

139. Charaxes solon sulphureus Rothschild, 1900 - Sulphur Black Rajah: SS recorded two individuals in Siju WS in May 2010. It is reportedly very rare in the Khasi Hills (Parsons \& Cantlie 1948). This subspecies is legally protected in India under Schedule II of the Wildlife (Protection) Act (Anonymous 1997).

140. Prothoe franck regalis Butler, 1885 - Regal Blue Begum: KK saw two specimens of this species in Jidung and Taidang streams in November 2009. The first one, seen on 18 November, was in dense evergreen forest on the community land that belongs to Gongrot Village. The second one, seen on 19 November, flew from the national park side of Taidang Stream into the evergreen forest on the Gongrot community land across the stream, but it could not be photographed. Our records of this subspecies from the Garo Hills are important for two reasons: (a) this is a significant range extension, and (b) it is legally protected in India under Schedule I of the Wildlife (Protection) Act (Anonymous 1997). This subspecies has so far been reported only from Upper Assam (Sibsagar to Margherita), Manipur, and N. Myanmar (Wynter-Blyth 1957; Gupta \& Mondal 2005). To our knowledge, there are no previous records either from the Khasi Hills or elsewhere west of Manipur. So this is a significant range extension of this very rare and presumably highly restricted subspecies by nearly $300 \mathrm{~km}$, over several mountain ranges. This record nearly doubles its global longitudinal range.

\section{Family Nymphalidae, Subfamily Cyrestinae}

141. Chersonesia risa risa Doubleday, 1848 - Oriental Common Maplet: This species occurred in both seasons, and it was commonly seen mudpuddling. It is reportedly uncommon in the Khasi Hills (Parsons \& Cantlie 1948).

142. Cyrestis cocles cocles Fabricius, 1787 - Thai Marbled Map Butterfly: We found the species to be common in the Gongrot area, although we also saw many individuals in Baghmara RF and Siju WS. Sometimes three or four specimens were seen mudpuddling together along forest streams, occasionally in company of other butterflies, but usually on their own. This species is generally scarce all over northeastern India but it can be locally common. Parsons \& Cantlie (1948) obtained "many specimens ... from Cherrapunjee professional collectors", but they never saw it in the Khasi Hills. This species is legally protected in India under Schedule II of the Wildlife (Protection) Act (Anonymous 1997).

143. Cyrestis thyodamas thyodamas Boisduval, 1836 - Oriental Map Butterfly: This species was common in the Gongrot area, especially during the pre-monsoon, when it was often seen mud-puddling. It is common in the Khasi Hills (Parsons \& Cantlie 1948).

\section{Family Nymphalidae, Subfamily Danainae}

144. Danaus chrysippus chrysippus Linnaeus, 1758 - Oriental Plain Tiger: We usually came across this species during the pre-monsoon when males were seen feeding from Heliotropium flowers that bloomed in abundance in fallow paddyfields. They were often in company of other danaines in these Heliotropium patches. The species is common in the Khasi Hills (Parsons \& Cantlie 1948).

145. Danaus genutia genutia Cramer 1779 - Oriental Striped Tiger: Similar to Danaus chrysippus, males were commonly encountered feeding from Heliotropium flowers in fallow fields and other open areas. The species is also common in the Khasi Hills, although it was earlier reported as Danais plexippus (Parsons \& Cantlie 1948), the name that applies to the North American Monarch Butterfly, not to the Indian Danaus genutia.

146. Euploea algea deione Westwood, 1848 
- Bengal Long-branded Blue Crow: All our sightings were of males feeding from Heliotropium flowers in the Halwa Atong and Siju areas, or in an all-male, multi-species congregation of Euploea that was seen in a patch of open forest near Gongrot. Our records have so far been exclusively from the pre-monsoon. The species is common in the Khasi Hills (Parsons \& Cantlie 1948).

147. Euploea core core Cramer, 1780 - Indian Common Crow: We found this species to be common during the pre-monsoon in Heliotropium patches in fallow paddyfields of Halwa Atong, usually with males of other Euploea. It was also commonly seen in the all-male, multi-species Euploea congregation in a patch of open forest near Gongrot. It is common in the Khasi Hills, and was previously reported as Euploea core vermiculata Butler, 1866 (Parsons \& Cantlie 1948), which is a synonym of the nominotypical subspecies (Talbot 1947; Kunte in prep.).

148. Euploea doubledayi doubledayi Felder \& Felder, 1865 - Sylhet Striped Black Crow: Another common species in the Gongrot area, details of our sightings were the same as for Euploea core core. The species is reportedly uncommon to rare in the Khasi Hills (Parsons \& Cantlie 1948).

149. Euploea klugii klugii Moore, 1858 - Blue King Crow: Our only record for the Garo Hills was from a single male that KK caught, photographed and released in a fallow paddyfield near Halwa Atong in November 2009. It is reportedly rare in the Khasi Hills (Parsons \& Cantlie 1948).

150. Euploea midamus rogenhoferi Felder \& Felder, 1865 - Assam Blue-spotted Crow: Our record for the Garo Hills is based on a single male that KK caught, photographed and released in the all-male, multi-species Euploea congregation in a patch of open forest near Gongrot in May 2009. It was said to be common in the Khasi Hills but Parsons \& Cantlie (1948) could collect only one specimen, which they reported as rogenhoferi's synonym, splendens Butler, 1866. This subspecies is legally protected in India under Schedule II of the Wildlife (Protection) Act (Anonymous 1997).

151. Euploea mulciber mulciber Cramer, 1777 - Bengal Striped Blue Crow: This was the most abundant Euploea in the Garo Hills, as is the case elsewhere in northeastern India. It was common in the Heliotropium patches of Halwa Atong and in the multi-species Euploea congregation near Gongrot, mentioned under Euploea core core. Males were also commonly seen mud-puddling and patrolling the streams and paddyfields at Baghmara RF. KT recorded 50 specimens in Nokrek NP. The species is also very common in the Khasi Hills (Parsons \& Cantlie 1948). Surprisingly, this very common subspecies is legally protected in India under Schedule IV of the Wildlife (Protection) Act (Anonymous 1997).

152. Euploea radamanthus radamanthus Fabricius, 1793 - Oriental Magpie Crow: This was another common Euploea in the Garo Hills, details of our sightings for this were the same as for Euploea core core. It is also common in the Khasi Hills, although previously reported as Euploea diocletiana diocletiana (Parsons \& Cantlie 1948), which is a synonym of radamanthus commonly used in older literature on Indian butterflies (Kunte in prep.).

153. Euploea sylvester hopei Felder \& Felder, 1865 - Cachar Double-branded Blue Crow: The details of our sightings of this species were the same as for Euploea algea deione. It is reportedly common in the Khasi Hills, although the subspecies was listed under Euploea harrisi (Parsons \& Cantlie 1948), which itself is now recognized as a subspecies of Euploea sylvester Fabricius, 1793 (Kunte in prep.).

154. Parantica aglea melanoides Moore, 1883 - Himalayan Glassy Tiger: One of the commonest danaines in our sampling, it was especially numerous at Nokrek NP in November 2009. It is also common in the Khasi Hills (Parsons \& Cantlie 1948).

155. Parantica melaneus plataniston Fruhstorfer, 1910 - Himalayan Chocolate Tiger: This species was less common than the last species, but it was also commoner at Nokrek NP in November 2009. It is also common in the Khasi Hills (Parsons \& Cantlie 1948).

156. Parantica sita sita Kollar, 1844 - Kashmir Chestnut Tiger: KT found this species to be common at mid-elevations at Nokrek NP in November 2009, although SS also had a single, unconfirmed record from Taidang stream near Gongrot in May 2010. This species is common in the Khasi Hills above $1,000 \mathrm{~m}$, where it was previously reported by its synonym, Danais tytia (Parsons \& Cantlie 1948).

157. Tirumala limniace exoticus Gmélin, 1790 Oriental Blue Tiger: A few males of this species were seen feeding from Heliotropium flowers at Halwa Atong, and in forest openings. It is common in the 
Khasi Hills (Parsons \& Cantlie 1948).

158. Tirumala septentrionis septentrionis Butler, 1874 - Oriental Dark Blue Tiger: The details of sightings of this species were the same as the previous species. It is also common in the Khasi Hills (Parsons \& Cantlie 1948).

\section{Family Nymphalidae, Subfamily Heliconiinae}

159. Argynnis hyperbius hyperbius Linnaeus, 1763 - Chinese Tropical Fritillary: This species is common in the Khasi Hills (Parsons \& Cantlie 1948) but must be relatively uncommon at lower elevations of Baghmara and Gongrot. We have recorded only two specimens so far, one from Gongrot and one from Nokrek NP, both in November 2009.

160. Cethosia biblis tisamena Fruhstorfer, 1912 - Himalayan Red Lacewing: This species occurs at slightly higher elevations compared to the next species, and we saw it almost exclusively at Nokrek NP, where it was very common. It is also very common at higher elevations in the Khasi Hills (Parsons \& Cantlie 1948).

161. Cethosia cyane cyane Drury, 1770 - Bengal Leopard Lacewing: We had numerous sightings of this common species, mostly from Gongrot and Baghmara RF during the pre-monsoon. It is also common in the Khasi Hills (Parsons \& Cantlie 1948).

162. Cirrochroa aoris aoris Doubleday, 1847 - Himalayan Large Yeoman: A common species that was frequently seen mud-puddling along evergreen forest streams at Gongrot. It is common in the Khasi Hills (Parsons \& Cantlie 1948).

163. Cirrochroa tyche mithila Moore, 1872 - Bengal Common Yeoman: A very common species, which we recorded along evergreen forest streams and paths, mainly at Gongrot. Males were often seen mudpuddling and they were also attracted to our baits of rotting crabs. Interestingly, it is reportedly uncommon in the Khasi Hills (Parsons \& Cantlie 1948).

164. Phalanta alcippe alcippoides Moore, 1900 - Himalayan Small Leopard: We recorded a total of six individuals of this species from Jidung Stream near Gongrot and from Karwani Stream in Baghmara RF. It is generally an uncommon species in northeastern India, and has been reported as such in the Khasi Hills (Parsons \& Cantlie 1948).

165. Phalanta phalantha phalantha Drury, 1773 - Oriental Common Leopard: We usually recorded this common species in drier, more open forests compared to the previous species, and also in abandoned jhum fields and sometimes around forest villages. It is also common in the Khasi Hills (Parsons \& Cantlie 1948).

166. Vagrans egista sinha Kollar, 1844 - Himalayan Vagrant: This species commonly occurs at slightly higher elevations, so it is not surprising that we have recorded only a single specimen in Baghmara RF so far. It is "not rare" in the Khasi Hills (Parsons \& Cantlie 1948).

167. Vindula erota erota Fabricius, 1793 - Thai Cruiser: This species can be locally common in evergreen forests but we have so far seen only four specimens mud-puddling along Taidang and Jidung streams near Gongrot. It is common in the Khasi Hills (Parsons \& Cantlie 1948).

\section{Family Nymphalidae, Subfamily Limenitidinae}

168. Athyma asura asura Moore, 1857 - Himalayan Studded Sergeant: We recorded one or two specimens of this species, always mud-puddling, on most of our field trips, although it was never common. This species is uncommon to rare throughout northeastern India, and it is reportedly rare in the Khasi Hills (Parsons \& Cantlie 1948). It is legally protected in India under Schedule II of the Wildlife (Protection) Act (Anonymous 1997).

169. Athyma cama cama Moore, 1857 - Himalayan Orange Staff Sergeant: We have recorded only two specimens of this species in the Garo Hills. It is "not rare" in the Khasi Hills (Parsons \& Cantlie 1948).

170. Athyma inara inara Doubleday, 1850 - Himalayan Color Sergeant: This species can be seasonally common in evergreen forests of northeastern India. We found it to be common in the GongrotBaghmara area, especially during the pre-monsoon when both sexes were frequently encountered along evergreen forest streams. Like other Athyma, males were usually encountered either mud-puddling or feeding from scats and rotting crabs. It is also common in the Khasi Hills (Parsons \& Cantlie 1948).

171. Athyma kanwa phorkys Fruhstorfer, 1912 - Northern Dot-dash Sergeant: Our Garo Hills record was based on a single specimen that SS photographed at Karwani in December 2009. This is a rare species in northeastern India, and it has apparently been recorded from the Khasi Hills based on a single male collected in November from Dauki (Cantlie 1952). 
172. Athyma perius perius Linnaeus, 1758 - Oriental Common Sergeant: We usually recorded males of this common species on hill-tops or ridges, patrolling areas nearby their vantage points on large rocks. It is reportedly common in the Khasi Hills (Parsons \& Cantlie 1948).

173. Athyma pravara acutipennis Fruhstorfer, 1906 - Assam Unbroken Sergeant: We recorded three males mud-puddling along evergreen forest streams near Gongrot during the post-monsoon. It is uncommon in the Khasi Hills (Parsons \& Cantlie 1948). The subspecies is legally protected in India under Schedule II of the Wildlife (Protection) Act (Anonymous 1997).

174. Athyma ranga ranga Moore, 1857 - Himalayan Blackvein Sergeant: This species was not uncommon along evergreen forest streams near Gongrot. It is uncommon in the Khasi Hills (Parsons \& Cantlie 1948), and is legally protected in India under Schedule II of the Wildlife (Protection) Act (Anonymous 1997).

175. Athyma selenophora bahula Moore, 1858 - Sylhet Staff Sergeant: We recorded two individuals at Gongrot and Karwani Stream. It is common in the Khasi Hills (Parsons \& Cantlie 1948).

176. Athyma zeroca zeroca Moore, 1872 - Khasi Small Staff Sergeant: An uncommon species in northeastern India, of which we have so far recorded only a single specimen at Gongrot. It is reportedly common in the Khasi Hills (Parsons \& Cantlie 1948).

177. Bassarona teuta teuta Doubleday, 1848 - Sylhet Banded Marquis: Our Garo Hills record was based on a single specimen that KT photographed on the Balpakram Plateau in November 2009. It is recorded as "fairly frequent" in the Khasi Hills (Parsons $\&$ Cantlie 1948). The species is legally protected in India under Schedule II of the Wildlife (Protection) Act (Anonymous 1997).

178. Bhagadatta austenia austenia Moore, 1872 - Khasi Grey Commodore: Both our records were from evergreen forests along Taidang Stream near Gongrot during the pre-monsoon. It is uncommon in the Khasi Hills (Parsons \& Cantlie 1948). The species is legally protected in India under Schedule II of the Wildlife (Protection) Act (Anonymous 1997).

179. Euthalia aconthea garuda Moore, 1857 - Northern Baron: So far we have recorded only two individuals - both females - of this species in the Garo Hills. It is "rather uncommon" in the Khasi Hills
(Parsons \& Cantlie 1948). Interestingly, this rather widespread and locally common subspecies is legally protected in India under Schedule II of the Wildlife (Protection) Act (Anonymous 1997).

180. Euthalia anosia anosia Moore, 1857 - Assam Grey Baron: SS recorded a single specimen of this rare species at Taidang stream near Gongrot in December 2009. This appears to be only the second record of this species from the Garo-Khasi Hills complex: the only previous record was midway (100-150 km) between Guwahati and Shillong, at $600 \mathrm{~m}$, in April 1933 (Cantlie 1952).

181. Euthalia lubentina lubentina Cramer, 1777 Chinese Gaudy Baron: We had three sporadic records, all females, from the Gongrot-Baghmara areas in both seasons. It is "not rare" in the Khasi Hills (Parsons \& Cantlie 1948). This species is legally protected in India under Schedule IV of the Wildlife (Protection) Act (Anonymous 1997).

182. Euthalia monina kesava Moore, 1859 - Assam Powdered Baron: We recorded several individuals, mostly males, especially during the post-monsoon at Karwani Stream. It is uncommon in the Khasi Hills (Parsons \& Cantlie 1948).

183. Euthalia phemius phemius Doubleday, 1848 - Sylhet White-edged Blue Baron: We have so far recorded two females in the Garo Hills in November 2009, one each from Me Cheng Chirang near Gongrot and Balpakram Plateau. The species is fairly common in the Khasi Hills (Parsons \& Cantlie 1948).

184. Euthalia telchinia Ménétriés, 1857 - Blue Baron: KT recorded a single female from Balpakram Plateau in November 2009. It is "rather rare" in the Khasi Hills (Parsons \& Cantlie 1948). It is legally protected in India under Schedule I of the Wildlife (Protection) Act. (Anonymous 1997).

185. Lebadea martha martha Fabricius, 1787 - Thai Knight: This was very common in both seasons throughout the forested areas of the Balpakram-SijuBaghmara landscape. We frequently encountered males patrolling neighborhoods of their vantage points on tall herbs and shrubs along forest paths and streams. The species is common in the Khasi Hills (Parsons \& Cantlie 1948).

186. Lexias cyanipardus cyanipardus Butler, 1868 - Sylhet Great Archduke: We had a single sighting of this large and spectacularly colorful species from an evergreen forest fragment among abandoned jhum 
fields near Gongrot, and four from the Balpakram Plateau, all from November 2009. All the specimens were males. The species is common in the Khasi Hills (Parsons \& Cantlie 1948). It is legally protected in India under Schedule II of the Wildlife (Protection) Act (Anonymous 1997).

187. Lexias dirtea khasiana Swinhoe, 1893 - Khasi Dark Archduke: This species was slightly commoner than the previous species, and most of our sightings were from the pre-monsoon rather than the postmonsoon. It is common in the Khasi Hills (Parsons \& Cantlie 1948). This subspecies is legally protected in India under Schedule II of the Wildlife (Protection) Act (Anonymous 1997).

188. Moduza procris procris Cramer, 1777 - Oriental Commander: This species was fairly common along forest streams near Gongrot, especially during the post-monsoon. It is also common in the Khasi Hills (Parsons \& Cantlie 1948).

189. Neptis hylas varmona Moore, 1872 - Indian Common Sailer: We found this species to be especially common in November 2009 in abandoned jhum fields, on paths and in other disturbed areas around Gongrot. It is also common in the Khasi Hills (Parsons \& Cantlie 1948).

190. Neptis nata/soma group - Sailer spp.: We must have seen several species from this group but we did not dissect male genitalia, without which species identification was impossible.

191. Neptis sappho astola Moore, 1872 - Himalayan Common Glider: Our record is based on a single specimen that was entangled in a spider web near Gongrot Village. The species is common at higher elevation in the Khasi Hills (Parsons \& Cantlie 1948).

192. Pantoporia hordonia hordonia Stoll, 1790 - Oriental Common Lascar: This species was common especially during the post-monsoon along forest streams and paths near Gongrot. It is common in the Khasi Hills (Parsons \& Cantlie 1948).

193. Pantoporia paraka paraka Butler, 1877 - Oriental Perak Lascar: KK saw one specimen each in evergreen forests along Jidung Stream near Gongrot and Karwani stream in Baghmara RF, both in November 2009. SS saw the third specimen at Karwani Stream in December 2009. The distribution of this species was given in older books as "Assam" (Evans 1932; Wynter-Blyth 1957), which presumably meant the
Naga-Manipur Hills and Cachar. Previous spot records are from Sylhet and Cachar in northeastern Bangladesh and southern Assam (Cantlie 1952), 100-200 km east of Balpakram. There are no previous records from the Khasi Hills (Parsons \& Cantlie 1948; Cantlie 1952), and our sightings seem to be the first records of the species this far west in northeastern India.

194. Parthenos sylvia gambrisius Fabricius, 1787 - Bengal Clipper: This species was common along evergreen forest streams both at Gongrot and Baghmara RF, especially during the pre-monsoon. It is also common in the Khasi Hills (Parsons \& Cantlie 1948). This subspecies is legally protected in India under Schedule II of the Wildlife (Protection) Act (Anonymous 1997).

195. Phaedyma columella ophiana Moore, 1872 - Sikkim Short-banded Sailer: We have only two records from the Gongrot area. The species is also reportedly rare in the Khasi Hills (Parsons \& Cantlie 1948).

196. Sumalia daraxa daraxa Doubleday, 1848 - Sylhet Green Commodore: This species is very common in the Khasi Hills (Parsons \& Cantlie 1948), and should be found in the Nokrek area.

197. Tanaeciajahnujahnu Moore, 1857 -Darjeeling Plain Earl: Our record is based on two males that KK saw at Me Cheng Chirang near Halwa Atong Village in November 2009. The species is "rather rare" in the Khasi Hills (Parsons \& Cantlie 1948).

198. Tanaecia julii appiades Ménétriés, 1857 - Changeable Common Earl: This species is generally common in northeastern India, and is reportedly common in the Khasi Hills (Parsons \& Cantlie 1948), so we are surprised that we have so far seen only one male in the Garo Hills.

199. Tanaecia lepidea lepidea Butler, 1868 - Himalayan Grey Count: We found this species to be very common in the Garo Hills in both seasons. Males, which made up the majority of our sightings, were usually seen along paths in evergreen forests. The species is also common in the Khasi Hills (Parsons \& Cantlie 1948). This species is legally protected in India under Schedule II of the Wildlife (Protection) Act (Anonymous 1997).

\section{Family Nymphalidae, Subfamily Nymphalinae}

200. Doleschallia bisaltide indica Moore, 1899 - Himalayan Autumn Leaf: This record is based on a 
single sighting in Baghmara RF in May 2010. The species is uncommon in the Khasi Hills (Parsons \& Cantlie 1948).

201. Hypolimnas bolina jacintha Drury, 1773 - Oriental Great Eggfly: Both sexes of this femalelimited Batesian mimic were very common in the Garo Hills, especially in open forests during the premonsoon in Gongrot and Baghmara areas. It is also common in the Khasi Hills (Parsons \& Cantlie 1948).

202. Hypolimnas misippus Linnaeus, 1764 - Danaid Eggfly: We recorded several individuals during the pre-monsoon, and this species will prove to be as common in the Garo and Khasi hills as elsewhere in the Oriental and African Regions. Interestingly, no specimens of this species seem to have been reported from the Garo-Khasi Hills before (Parsons \& Cantlie 1948). Surprisingly, this common and widespread species is legally protected in India under Schedule I of the Wildlife (Protection) Act (Anonymous 1997).

203. Junonia almana almana Linnaeus, 1758 - Oriental Peacock Pansy: We found this species to be extremely abundant in fallow paddyfields and along edges of evergreen forests. In fact, it was the most numerous species in our quantitative sampling (Fig. 1). This was especially true during the pre-monsoon, although it was present in both seasons. It is also common in the Khasi Hills (Parsons \& Cantlie 1948).

204. Junonia atlites atlites Linnaeus, 1763 - Oriental Grey Pansy: This was also a common Junonia in our sampling and we found it co-occurring with the previous species especially in fallow paddyfields.

205. Junonia hierta hierta Fabricius, 1798 - Oriental Yellow Pansy: This species was less numerous than the previous two Junonia, but still very common in and around agricultural fields and other highly disturbed areas. It is common in the Khasi Hills (Parsons \& Cantlie 1948).

206. Junonia iphita iphita Cramer, 1779 - Oriental Chocolate Pansy: This species was very common in all kinds of forested areas, especially along forest edges and in natural openings, but also around human habitations. It is common in the Khasi Hills (Parsons \& Cantlie 1948).

207. Junonia lemonias lemonias Linnaeus, 1758 - Chinese Lemon Pansy: This was the second-most numerous Junonia in our sampling. We found it to be common along major roads and around human habitations, including in agricultural fields. It is very common in the Khasi Hills (Parsons \& Cantlie 1948).

208. Junonia orithya ocyale Hübner, 1816 - Dark Blue Pansy: Our record is based on a single specimen that KT photographed on the Balpakram Plateau in November 2009. It is very common in the Khasi Hills (Parsons \& Cantlie 1948).

209. Kallima inachus inachus Boisduval, 1836 - Himalayan Orange Oakleaf: We found this species to be common along forest streams and inside evergreen forests, especially near Gongrot during the pre-monsoon. It is also common in the Khasi Hills (Parsons \& Cantlie 1948).

210. Kaniska canace canace Linnaeus, 1763 - Chinese Blue Admiral: KT recorded three individuals from Balpakram Plateau in November 2009. The species is common in the Khasi Hills (Parsons \& Cantlie 1948).

211. Symbrenthia hypselis cotanda Moore, 1874 - Himalayan Spotted Jester: We have a single record from Nokrek NP. It is uncommon in the Khasi Hills (Parsons \& Cantlie 1948).

212. Symbrenthia lilaea khasiana Moore, 1874 - Khasi Common Jester: We commonly encountered this species along evergreen forest streams near Gongrot, either mud-puddling or basking in occasional sunny spots along paths under the canopy. It is also common in the Khasi Hills (Parsons \& Cantlie 1948).

213. Symbrenthia silana de Nicéville, 1885 - Scarce Jester: KT recorded a single specimen of this rare species mud-puddling in Nokrek NP. This sighting has previously been reported in literature (Kunte 2010). Since then, we have come across another old record of this species from this area, a specimen collected $60 \mathrm{~km}$ away from Shillong, halfway between Guwahati and Shillong, in April 1933 (Cantlie 1952). This species is legally protected in India under Schedule I of the Wildlife (Protection) Act (Anonymous 1997).

214. Vanessa cardui cardui Linnaeus, 1758 - Painted Lady: This almost globally distributed species occurs seasonally in many parts of India, and it can be locally common in the right season. We have so far recorded only a single specimen feeding from Heliotropium flowers in a fallow paddyfield in Halwa Atong. The species is reportedly very common in the Khasi Hills (Parsons \& Cantlie 1948).

215. Vanessa indica indica Herbst, 1794 - Himalayan Red Admiral: BS recorded several specimens from Rongreng Stream, Hangsapal. It is 
common in the Khasi Hills (Parsons \& Cantlie 1948).

\section{Family Nymphalidae, Subfamily Pseudergolinae}

216. Dichorragia nesimachus nesimachus Doyère, 1840 - Himalayan Constable: Our record is based on a single, slightly worn specimen that KK and RL photographed on the sandy bed of Karwani Stream in Baghmara RF in May 2008. It was feeding from a small carnivore scat along with Charaxes bernardus hierax on a rainy day. It is reportedly uncommon in the Khasi Hills (Parsons \& Cantlie 1948).

217. Stibochiona nicea nicea Gray, 1846 - Himalayan Popinjay: We recorded 14 specimens of this species in both seasons, although majority of sightings (10 individuals) were from November 2009 from evergreen forest streams near Gongrot and bamboo patches in mixed semi-evergreen forests around Halwa Atong. The species is also common in the Khasi Hills (Parsons \& Cantlie 1948).

\section{Family Nymphalidae, Subfamily Satyrinae}

218. Amathuxidia amythaon amythaon Doubleday, 1847 - Sylhet Koh-i-Noor: This species is generally rare at its northwestern range margin in northeastern India. Our Garo Hills record is based on four specimens. The first one was a piece of right forewing that KK discovered in a dry streambed in evergreen forest at Siju WS in November 2009. The remaining three specimens were seen by SS, RL and GA at Karwani in Baghmara RF in May 2010. Only four specimens seem to have been reported from the Khasi Hills before (Parsons \& Cantlie 1948; Cantlie 1952). This species is legally protected in India under Schedule II of the Wildlife (Protection) Act (Anonymous 1997).

219. Discophora sondaica zal Westwood, 1851 - Indian Common Duffer: This is a fairly common species despite its crepuscular habits. We recorded five specimens, all males, in both seasons, and mostly inside evergreen forests at Gongrot. It has been reported from the Khasi Hills before but seldom seen there (Parsons \& Cantlie 1948).

220. Elymnias hypermnestra undularis Drury, 1773 - Wavy Common Palmfly: A common species, males of which were especially numerous along Karwani Stream in Baghmara RF in May 2008. It is also common in the Khasi Hills (Parsons \& Cantlie 1948). Females of this sex-limited Batesian mimic are superb mimics of Danaus genutia in flight.
221. Elymnias malelas malelas Hewitson, 1863 - Bengal Spotted Palmfly: Our record is based on a single specimen that BS photographed at Karwani, Baghmara RF. The species is common in the Khasi Hills (Parsons \& Cantlie 1948).

222. Elymnias nesaea timandra Wallace, 1869 - Sylhet Tiger Palmfly: KT recorded one individual on Balpakram Plateau in November 2009, and SS recorded one from Siju WS in December 2009 and another outside Samrakshan's office in Baghmara town in May 2010. The species is not rare in the Khasi Hills (Parsons \& Cantlie 1948).

223. Elymnias peali Wood-Mason, 1883 - Brahmaputra Palmfly: This is a slight range extension of the species, and the first record from the Garo Hills. It has previously been reported from upper Assam (Wynter-Blyth 1957), and from Namdapha NP in eastern Arunachal Pradesh (Kunte et al. 2011). The nearest record to the Garo Hills was that of D. Sanders, who took a specimen approximately $60 \mathrm{~km}$ from Shillong on Guwahati Road in April 1933 (Cantlie 1952). That was approximately $100-150 \mathrm{~km}$ from the present locality in Balpakram NP. As far as we know, the previous westernmost record north of the Brahmaputra River was that of KK, who recorded a male in February 2001 from near Seijusa in Pakke NP in western Arunachal Pradesh (unpub.). Our following records from the Garo Hills delineate the westernmost range of this species south of the Brahmaputra River. KK photographed a male from Me Cheng Chirang near Halwa Atong on 20 November 2009. SS recorded one individual from Panda Chirring in December 2009 and another at Karwani Stream in May 2010. The species is generally considered very rare throughout its range, so these three sightings and the range extensions are particularly important. The species is endemic to lowland evergreen forests in and near the Brahmaputra River basin, and it is legally protected in India under Schedule I of the Wildlife (Protection) Act (Anonymous 1997).

224. Ethope himachala Moore, 1857 - Dusky Diadem: This was the third-commonest satyrine and among the most numerous species in our sampling. We saw a total of 61 individuals, nearly $2 / 3^{\text {rd }}$ of them in November 2009 from Taidang, Jidung and Me Cheng Chirang in the Gongrot-Halwa Atong landscape and from the Karwani Stream in the Baghmara RF. Most of the individuals, of both sexes, were seen basking and 
chasing each other among somewhat dense vegetation along evergreen forest streams. The species is "not rare" in the Khasi Hills (Parsons \& Cantlie 1948).

225. Faunis canens arcesilas Stichel, 1933 - Thai Common Faun: A fairly common species that we encountered in dense undergrowth along paths and small streams under closed canopy of evergreen forests near Gongrot. It is "not rare" in the Khasi Hills (Parsons \& Cantlie 1948).

226. Faunis eumeus assama Westwood, 1858 - Assam Large Faun: We recorded this species based on two specimens that GA, RL and SS saw in Taidang Stream near Gongrot in May 2010. It is "not rare" in the Khasi Hills (Parsons \& Cantlie 1948).

227. Lethe chandica flanona Fruhstorfer, 1911 - Assam Angled Red Forester: We had two records of this species: a male from Rani Miksuram Stream near Halwa Atong in November 2009, and another male from Nokrek NP in November 2009. It is "scarce" in the Khasi Hills (Parsons \& Cantlie 1948).

228. Lethe confusa gambara Fruhstorfer, 1911 - Assam Banded Treebrown: We found this species to be common, although all our sightings were from November 2009 from the Gongrot-Halwa Atong area, and from Siju WS. It is also common in the Khasi Hills (Parsons \& Cantlie 1948).

229. Lethe europa niladana Fruhstorfer, 1911 - Himalayan Bamboo Treebrown: Not uncommon, and our sightings were from both seasons and from multiple localities. It is reportedly uncommon in the Khasi Hills (Parsons \& Cantlie 1948).

230. Lethe mekara zuchara Fruhstorfer, 1911 - Assam Common Red Forester: A common species that we usually found mud-puddling or visiting animal droppings along various evergreen forest streams near Gongrot and Halwa Atong villages and in Baghmara RF. It was recorded in both seasons . It is "not rare" in the Khasi Hills (Parsons \& Cantlie 1948).

231. Lethe rohria rohria Fabricius, 1787 - Himalayan Common Treebrown: Our record is based on a single sighting by KT at Nokrek NP in November 2009. The species is common in the Khasi Hills (Parsons \& Cantlie 1948).

232. Lethe verma sintica Fruhstorfer, 1911 - East Himalayan Straight-banded Treebrown: We recorded two specimens from Gongrot in May 2010, and one specimen from Nokrek NP in November 2009.

233. Lethe vindhya vindhya Felder, 1859 - Assam
Black Forester: Our record of this uncommon species is based on two sightings: one by SS at Panda Chirring in December 2009, and the other by BS near Gongrot. The species is uncommon in the Khasi Hills (Cantlie 1952).

234. Melanitis leda leda Linnaeus, 1758 - Oriental Common Evening Brown: This was the secondcommonest species in our sampling, with a total of 157 individuals (Fig. 1). Specimens of both sexes were commonly encountered in both seasons in evergreen and semi-evergreen forest undergrowth in all localities surveyed. The wet season form was more prevalent in May while the dry season form was more prevalent in November. It is also very common in the Khasi Hills (Parsons \& Cantlie 1948).

235. Melanitis phedima bela Moore, 1857 - Bengal Dark Evening Brown: This was overall a common species, but much less common compared to the last species, with which it shared its habitat. It is uncommon in the Khasi Hills (Parsons \& Cantlie 1948).

236. Melanitis zitenius zitenius Herbst, 1796 - Himalayan Great Evening Brown: This was the least common Melanitis in our sampling. We had three sightings, one near Gongrot and two from Siju WS (one in each season). The species is not rare in the Khasi Hills (Parsons \& Cantlie 1948). It is legally protected in India under Schedule II of the Wildlife (Protection) Act (Anonymous 1997).

237. Mycalesis anaxias aemate Fruhstorfer, 1911 - Indo-Chinese White-bar Bushbrown: This was commonly seen in dense evergreen forests at Gongrot, Baghmara RF and Siju WS. It was recorded in both seasons. It is uncommon in the Khasi Hills (Parsons \& Cantlie 1948). This subspecies is legally protected in India under Schedule II of the Wildlife (Protection) Act (Anonymous 1997).

238. Mycalesis francisca sanatana Moore, 1857 - Himalayan Lilacine Bushbrown: KT photographed a single specimen at Nokrek NP in November 2009. This species is uncommon at mid-elevations in the Khasi Hills (Parsons \& Cantlie 1948).

239. Mycalesis intermedia Moore, 1892 - Intermediate Bushbrown: We had three records of this species based on two individuals seen at Taidang/ Jidung streams near Gongrot and one individual seen at Siju WS, all in November 2009. This species has previously been listed from the Khasi Hills by its synonym, Mycalesis khasia Evans, 1912, but without 
any details (Cantlie 1952). Calysisme intermedia Moore, 1892 is the oldest available name for this taxon (Talbot 1947; Larsen 2004; Kunte in prep.).

240. Mycalesis malsarida Butler, 1868 - Plain Bushbrown: KK saw seven specimens in Nov. 2009 in evergreen forests along the Taidang and Jidung streams near Gongrot and at Karwani Stream in Baghmara RF. It is reportedly "not rare" and "seems scarce" from the Khasi Hills (Parsons \& Cantlie 1948). This species is protected in India under Schedule II of the Wildlife (Protection) Act (Anonymous 1997).

241. Mycalesis mineus mineus Linnaeus, 1758 - Chinese Dark-branded Bushbrown: This is usually common in most forested regions of India, but we have so far recorded only three individuals from disturbed forests around Gongrot. It is very common in the Khasi Hills (Parsons \& Cantlie 1948).

242. Mycalesis perseus blasius Fabricius, 1798 - Himalayan Common Bushbrown: This was uncommon in evergreen, semi-evergreen and moist deciduous forests at Me Cheng Chirang near Halwa Atong, in Baghmara RF and Siju WS. The species was initially overlooked and therefore not reported from the Khasi Hills (Parsons \& Cantlie 1948), but a few specimens were subsequently collected (Cantlie 1952).

243. Mycalesis spp. - Bushbrowns: Several Mycalesis females with distinct phenotypes were seen at all the localities in both seasons but were not collected, and therefore cannot be identified by any means now.

244. Mycalesis visala visala Moore, 1857 - Indian Long-branded Bushbrown: We have so far found a single specimen of this species in November 2009 near Gongrot. This is surprising because the species is usually common, and is often among the most abundant Mycalesis in places such as Manas and Namdapha national parks in northeastern India. This was reported as "probably uncommon" in the Khasi Hills based on only a few specimens collected (Parsons \& Cantlie 1948).

245. Orsotriaena medus medus Fabricius, 1775 - Oriental Medus Brown: This was common in open and/or disturbed forest areas such as around large streams and at forest edges. Both the wet and dry season forms, which differ greatly, were seen in both seasons. It is also very common in the Khasi Hills (Parsons \& Cantlie 1948).
246. Thaumantis diores diores Doubleday, 1845 - Assam Jungleglory: This was fairly common, but most of our records (five out of six) were from November 2009, so the post-monsoon seems to be its peak flight period in the Garo Hills. It is also common in the Khasi Hills (Parsons \& Cantlie 1948).

247. Ypthima baldus satpura Evans, 1923 - Satpuda Common Five-ring: This was among the most abundant species in the Garo Hills, where we recorded it in both seasons at all the localities surveyed. It was especially common in disturbed habitats such as forest edges, agricultural fields, orchards, and around human habitations. It is also very common in the Khasi Hills (Parsons \& Cantlie 1948). There should be some Ypthima affectata Elwes \& Edwards, 1893, the Eastern Five-ring, among our presumed Ypthima baldus satpura. However, we did not collect and dissect any Ypthima, so we are not sure about all the other species of Ypthima that we may have encountered. Cantlie had found Ypthima affectata to be "A speciality [sic] of the Khasi Hills, discoverable among one's supposed baldus." However, Ypthima baldus satpura usually outnumbers any other Ypthima in this area, just as in forested regions elsewhere in India.

248. Ypthima huebneri Kirby, 1871 - Common Four-ring: Another common Ypthima species, which was found in more or less the same habitats as the previous species but was comparatively less frequently seen. It is also common in the Khasi Hills (Parsons \& Cantlie 1948).

\section{Family Papilionidae (Swallowtail Butterflies) Subfamily Papilioninae}

249. Atrophaneura varuna astorion Westwood, 1842 - Sylhet Common Batwing: This was common in both seasons along wide, open forest streams, where males were encountered mud-puddling. Females were usually seen in denser forests and along evergreen forest paths, where their larval host plant was found. It has previously been recorded as common in the Khasi Hills (Parsons \& Cantlie 1948).

250. Byasa sp. - Windmill sp.: KT recorded a single individual of Byasa, which could not be photographed and hence we have not been able to identify it to species level. The image included in image 15 for Byasa is merely for illustration purposes, it does not represent a specimen seen in the Garo Hills. This image was taken in Ngengpui WS in Mizoram, and is of Byasa 
dasarada dasarada Moore, 1857, the East Himalayan Great Windmill.

251. Graphium agamemnon agamemnon Linnaeus, 1758 - Oriental Tailed Jay: This was fairly common in evergreen forests, and was recorded in both seasons. It is uncommon in the Khasi Hills (Parsons \& Cantlie 1948).

252. Graphium agetes agetes Westwood, 1843 - Assam Four-bar Swordtail: Our five records were from May 2010 from Karwani, Bhawanipur and Panda Chirring in the Baghmara RF. It is "not rare" at Dauki near the Khasi Hills (Parsons \& Cantlie 1948).

253. Graphium antiphates pompilius Fabricius, 1787 - Indo-Chinese Five-bar Swordtail: This species was commonly seen mud-puddling along evergreen forest streams in all the localities surveyed around Baghmara. Most of the sightings were from May 2010, but two were from November 2009. It is also common in the Khasi Hills (Parsons \& Cantlie 1948).

254. Graphium aristeus anticrates Doubleday, 1846 - Assam Chain Swordtail: SS recorded one specimen from Siju WS in May 2010, mud-puddling in the company of other Graphium and Papilio. The Khasi Hills records were based on two specimens taken in April (Parsons \& Cantlie 1948). This subspecies is legally protected in India under Schedule II of the Wildlife (Protection) Act (Anonymous 1997).

255. Graphium cloanthus cloanthus Westwood, 1841 - Himalayan Glassy Bluebottle: Our single record was from Balpakram Plateau in November 2009. The picture included in image 15 is not from the Garo Hills, it is actually from the Namdapha NP, used here as a reference for this species. The specimen seen on Balpakram Plateau could not be photographed, so this record needs further confirmation. The species is common at higher elevations in the Khasi Hills (Parsons \& Cantlie 1948).

256. Graphium doson axion Felder \& Felder, 1864 - Himalayan Common Jay: A very common species in evergreen and semi-evergreen forests, whose main flight period in the Garo Hills seems to be the pre-monsoon, to which all our sightings have been restricted so far. It is also common in the Khasi Hills (Parsons \& Cantlie 1948).

257. Graphium eurypylus cheronus Fruhstorfer, 1903 - Indo-Chinese Great Jay: This was much less common than the previous species, and was seen mostly in denser evergreen forests. It is uncommon in the Khasi Hills (Parsons \& Cantlie 1948).

258. Graphium macareus lioneli Fruhstorfer, 1902 - Khasi Lesser Zebra: Our record is based on a single specimen that SS photographed at Panda Chirring in May 2010. Males of this species are reportedly common in the Khasi Hills (Parsons \& Cantlie 1948).

259. Graphium sarpedon sarpedon Linnaeus, 1758 - Oriental Common Bluebottle: This was a very common species, especially during the pre-monsoon. Males were usually encountered mud-puddling along evergreen forest streams near Gongrot and Baghmara RF.

260. Graphium xenocles xenocles Doubleday, 1842 - Sylhet Great Zebra: This is very similar to Graphium macareus lioneli, with which it shared the habitat but was encountered more frequently in the Garo Hills. It is uncommon in the Khasi Hills (Parsons \& Cantlie 1948).

261. Lamproptera curius curius Fabricius, 1787 - Thai White Dragontail: This was a common species, whose males were usually seen mud-puddling along streams in evergreen forests in the Gongrot area. It is also common in the Khasi Hills (Parsons \& Cantlie 1948).

262. Losaria coon cacharensis Butler, 1885 - Cachar Common Clubtail: This is generally a very rare species in northeastern India but reportedly common in the Garo Hills and in Cachar, SE Assam (Parsons \& Cantlie 1948). Our Garo Hills record was based on a single specimen seen by KK on 2 May 2008 at Rani Miksuram Stream near Halwa Atong. It could not be photographed, however, so we have used, as a reference, picture in image 16 of an old specimen from the Khasi Hills.

263. Meandrusa payeni evan Doubleday, 1845 - Sikkim Yellow Gorgon: Our Garo Hills record is based on a single female photographed by BS at Halwa Ambeng near Gongrot in September 2008. The species is apparently common in the Khasi Hills (Parsons \& Cantlie 1948).

264. Pachliopta aristolochiae aristolochiae Fabricius, 1775 - Indian Common Rose: This species was, along with Papilio polytes romulus, the most abundant papilionid in our sampling. We recorded 69 individuals in both seasons, although the species was much commoner during the summer. All our sightings were from rural areas and all types of open forest throughout the Garo Hills. 
265. Papilio alcmenor alcmenor Felder \& Felder, 1864 - Khasi Redbreast: Our record is based on KT's single sighting from Balpakram Plateau in November 2009. The species is reportedly very common in the Khasi Hills (Parsons \& Cantlie 1948).

266. Papilio bianor gladiator Fruhstorfer, 1902 - Indo-Chinese Common Peacock: Our record is based on a single sighting from Dabit, Baghmara RF, in May 2010. Another subspecies, Papilio bianor ganesa Doubleday, 1842, has previously been reported from the Khasi Hills as common (Parsons \& Cantlie 1948). However, ganesa occurs north of the Brahmaputra River, and gladiator is the correct subspecific name for the populations south of the Brahmaputra River.

267. Papilio castor castor Westwood, 1842 - Khasi Common Raven: This was a common species, whose males and females were seen along evergreen forest streams near Gongrot, and in Baghmara RF and Siju WS. However, all our sightings have so far been only from the pre-monsoon, which seems to be its peak flight period in the Garo Hills. The species is reportedly uncommon in the Khasi Hills, especially the female (Parsons \& Cantlie 1948).

268. Papilio clytia clytia Linnaeus, 1758 - Oriental Common Mime: This species was common along evergreen forest streams. This is a Batesian mimic with two forms in northeastern India: form dissimils mimics Tirumala, and form clytia mimics brown-andwhite Euploea. We recorded both the forms in the Garo Hills, although form dissimilis was more frequently seen. The species has previously been reported to be common in the Khasi Hills and at Dauki (Parsons \& Cantlie 1948).

269. Papilio demoleus demoleus Linnaeus, 1758 - Northern Lime Butterfly: We encountered this species commonly around human habitations, in orchards, and in open forests. It is very common in the Khasi Hills (Parsons \& Cantlie 1948).

270. Papilio helenus helenus Linnaeus, 1758 - Oriental Red Helen: This was a very common species, whose males were often seen mud-puddling with other Papilio along evergreen forest streams. It is also common in the Khasi Hills (Parsons \& Cantlie 1948).

271. Papilio memnon agenor Linnaeus, 1758 - Continental Great Mormon: We recorded 29 individuals, most of them male, during both the seasons. All the males that we saw belonged to the form agenor, and all the females belonged to the form alcanor, which mimics Pachliopta aristolochiae. The species has previously been reported as common in the Garo and Khasi Hills (Parsons \& Cantlie 1948).

272. Papilio nephelus chaon Westwood, 1845 - Assam Yellow Helen: In the Garo Hills, habits and habitat of this species were similar to Papilio helenus helenus, and it was equally abundant. It is also common in the Khasi Hills (Parsons \& Cantlie 1948).

273. Papilio paradoxa telearchus Hewitson, 1852 - Khasi Great Blue Mime: Our record is based on a single individual that SS photographed near Karwani in Baghmara RF in May 2010. The species is reportedly rare in the Khasi Hills (Parsons \& Cantlie 1948). This subspecies is legally protected in India under Schedule II of the Wildlife (Protection) Act (Anonymous 1997).

274. Papilio paris paris Linnaeus, 1758 - Chinese Paris Peacock: We recorded 12 individuals, one each at Baghmara RF and Balpakram NP during the summer, and 10 at Nokrek NP during post-monsoon. Males were seen mud-puddling and patrolling along forest streams and ridges. The species is widely distributed in the Oriental Region, common throughout the Himalaya and northeastern India, and has been reported from the Khasi Hills before (Parsons \& Cantlie 1948).

275. Papilio polytes romulus Cramer, 1775 - Indian Common Mormon: We recorded 69 individuals in both seasons, making this one of the commonest species in our sampling (Fig. 1), although it was much more abundant during the summer. All our records were from rural/agricultural landscapes as well as inside all forest types at all the sampled localities. The female form stichius (also known as the form polytes outside taxonomic literature) is an excellent mimic of Pachliopta aristolochiae, and all the females that we came across in the Garo Hills belonged to this form. We have not yet come across the non-mimetic female form cyrus or the Pachliopta hector-mimicking female form romulus in the Garo Hills. The species is also very common in the Khasi Hills (Parsons \& Cantlie 1948)

276. Papilio protenor euprotenor Fruhstorfer, 1908 - East Himalayan Spangle: KK and RL recorded only two male specimens in May 2008.

277. Troides helena cerberus Felder \& Felder, 1865 - Khasi Common Birdwing: We recorded 13 specimens of both sexes from Baghmara RF and Balpakram NP 
in both seasons. These were usually seen flying in the canopy along forest streams and edges. The species is reportedly common in the Khasi Hills (Parsons \& Cantlie 1948).

\section{Family Pieridae (Whites And Yellows) Subfamily Coliadinae}

278. Catopsilia pomona pomona Fabricius, 1775 - Oriental Common Emigrant: This was very common near human habitations and in deciduous forest patches, and most of our sightings were from the pre-monsoon. It is also common in the Khasi Hills (Parsons \& Cantlie 1948).

279. Catopsilia pyranthe pyranthe Linnaeus, 1758 - Oriental Mottled Emigrant: SS recorded this from Karwani in December 2009. It is reportedly common in the Khasi Hills (Parsons \& Cantlie 1948).

280. Dercas verhuelli doubledayi Moore, 1905 - Indo-Chinese Tailed Sulphur: This was common during the pre-monsoon, and all our sightings were from Taidang-Jidung streams near Gongrot. The species is reportedly rare in the Khasi Hills (Parsons \& Cantlie 1948).

281. Eurema andersonii jordani Corbet \& Pendlebury, 1932 - Sikkim One-spot Grass Yellow: This species seems generally uncommon, but KK saw a large group (15 individuals) mud-puddling in Taidang Stream, and then two at Siju WS, all in November 2009. The species was not reported from the Khasi Hills (Parsons \& Cantlie 1948).

282. Eurema blanda silhetana Wallace, 1867 - Sylhet Three-spot Grass Yellow: This was very common along evergreen forest streams and paths near Gongrot and in Baghmara RF, especially during the post-monsoon. Males were often seen mud-puddling in small groups. The species is also common in the Khasi Hills (Parsons \& Cantlie 1948).

283. Eurema hecabe hecabe Linnaeus, 1758 - Oriental Common Grass Yellow: This species was also very common, usually in the same habitats as the previous species, although it also occurred in more open forests and around human habitations. It was more common during the pre-monsoon. The species is also common in the Khasi Hills (Parsons \& Cantlie 1948).

284. Gandaca harina assamica Moore, 1906 - Assam Tree Yellow: This was common along evergreen forest streams near Gongrot, where males were usually encountered mud-puddling. It is also common in the Khasi Hills (Parsons \& Cantlie 1948).

\section{Family Pieridae, Subfamily Pierinae:}

285. Appias albina darada Felder \& Felder, 1865 - Indian Common Albatross: Our record is based on four males seen mud-puddling and feeding from flowers along evergreen forest streams and forest edges near Gongrot and on the Balpakram Plateau. It is reportedly rare in the Khasi Hills (Parsons \& Cantlie 1948). This subspecies is legally protected in India under Schedule II of the Wildlife (Protection) Act (Anonymous 1997).

286. Appias indra indra Moore, 1857 - Himalayan Plain Puffin: We found this species to be common in mud-puddling assemblages in Taidang and Jidung streams near Gongrot. All our sightings have only been from the pre-monsoon so far, which seems to be its peak flight period at this elevation in the Garo Hills. It is uncommon in the Khasi Hills (Parsons \& Cantlie 1948).

287. Appias lalage lalage Doubleday, 1842 - Himalayan Spot Puffin: This species prefers midelevation evergreen forests throughout its range (Kunte et al. 2008), and our only sighting was from mid-elevations at Nokrek NP. It is common in the mid-elevation forests in the Khasi Hills (Parsons \& Cantlie 1948).

288. Appias lyncida eleonora Boisduval, 1836 - Indo-Chinese Chocolate Albatross: This species was abundant in both seasons, frequenting deciduous forest patches and large openings in evergreen and semievergreen forests in all the localities surveyed by us. It is common in the Khasi Hills, although previously reported by its synonym, Appias lyncida hippoides Moore, 1881 (Parsons \& Cantlie 1948).

289. Appias olferna Swinhoe, 1890 - Eastern Striped Albatross: Our record is based on four individuals seen in Baghmara RF in May 2010. It is uncommon in the Khasi Hills (Parsons \& Cantlie 1948). The taxon was previously treated as a subspecies of Appias libythea Fabricius, 1775, the Western Striped Albatross.

290. Cepora nadina nadina Lucas, 1852 - Khasi Lesser Gull: This was common in the Gongrot and Baghmara RF areas, especially in evergreen forests during the pre-monsoon. It is also common in the Khasi Hills (Parsons \& Cantlie 1948).

291. Cepora nerissa nerissa Fabricius, 1775 - 
Chinese Common Gull: This species occurred in much more open and drier habitats than the previous species, and we have only two sightings from the pre-monsoon, one each from Baghmara and Gongrot areas. It is common in the Khasi Hills (Parsons \& Cantlie 1948).

292. Delias agostina agostina Hewitson, 1852 - Sikkim Yellow Jezebel: SS recorded a single individual at Baghmara in December 2009. The species is uncommon in the Khasi Hills (Parsons \& Cantlie 1948).

293. Delias belladonna lugens Jordan, 1925 - Lushai Hill Jezebel: SS saw two specimens in flight, one in December 2009 at Simsang trail and the other near Gongrot in May 2010. However, these could not be photographed, and our sightings need further confirmation. It is uncommon in the Khasi Hills (Parsons \& Cantlie 1948).

294. Delias descombesi descombesi Boisduval, 1836 - Vietnamese Red-spot Jezebel: This species was common during the post-monsoon, when we saw five specimens at the edges of abandoned agricultural fields overgrown with flowering herbs and shrubs along the Rongrok Stream near Halwa Atong. Then several more were seen in Nokrek NP and on Balpakram Plateau. The species is common in the Khasi Hills (Parsons \& Cantlie 1948).

295. Delias hyparete indica Wallace, 1867 - Himalayan Painted Jezebel: We had few, sporadic sightings of this uncommon species at Gongrot and in Baghmara RF in November and December 2009. It is also uncommon in the Khasi Hills (Parsons \& Cantlie 1948).

296. Delias pasithoe pasithoe Linnaeus, 1767 - Chinese Red-base Jezebel: This species was common, especially during the post-monsoon, in nearly all the localities surveyed by us. Most of the sightings were of mud-puddling males in evergreen forests. It is also common in the Khasi Hills (Parsons \& Cantlie 1948).

297. Hebomoia glaucippe glaucippe Linnaeus, 1758 - Oriental Great Orange-tip: This species was common in mud-puddling assemblages in open forests, especially during the pre-monsoon. It is common in the Khasi Hills (Parsons \& Cantlie 1948).

298. Ixias pyrene familiaris Butler, 1874 - East Himalayan Yellow Orange-tip: This was common especially in open forests and along the edges of evergreen forests near Gongrot. It is common in the Khasi Hills (Parsons \& Cantlie 1948).
299. Leptosia nina nina Fabricius, 1793 - Oriental Psyche: We had relatively few sightings near human habitations and in orchards of this otherwise common and widely distributed species. It is very common in the Khasi Hills (Parsons \& Cantlie 1948).

300. Pareronia hippia Fabricius, 1787 - Indian Wanderer: SS had a single record of this species from Karwani in December 2009. This species has not been recorded from the Khasi Hills before (Parsons \& Cantlie 1948), and our sighting needs confirmation since this specimen could not be photographed. Another closely related species, Pareronia avatar Moore, 1857, the Pale Wanderer, has previously been reported from the Garo Hills, but not from the Khasi Hills (Parsons \& Cantlie 1948; Cantlie 1952).

301. Pieris canidia indica Evans, 1926 - Indian Cabbage White: SS recorded a single individual near Gongrot in May 2010. The species is common in the Khasi Hills (Parsons \& Cantlie 1948).

\section{Family Riodinidae (Metalmarks) Subfamily Nemeobiinae}

302. Abisara bifasciata suffusa Moore, 1882 - Indian Double-banded Judy: KT recorded a single individual from Balpakram Plateau in November 2009. This species does not seem to have been recorded from the Khasi Hills before (Parsons \& Cantlie 1948).

303. Abisara fylla Westwood, 1851 - Dark Judy: This was common in Nokrek NP. It is also common in the Khasi Hills (Parsons \& Cantlie 1948).

304. Abisara neophron neophron Hewitson, 1861 - Khasi Tailed Judy: This is included based on two individuals, one recorded by BS in the Gongrot area and another from Nokrek NP by KT. The species is reportedly uncommon in the Khasi Hills (Parsons \& Cantlie 1948).

305. Dodona longicaudata de Nicéville, 1881 - Long-tailed Punch: KT photographed a single individual on Balpakram Plateau in November 2009. This is the westernmost record for this species, perhaps as much as $200 \mathrm{~km}$ west of its previously known range in the Khasi Hills. This species is generally very rare throughout its range, and reportedly so in the Khasi Hills from where it is known only from three or four specimens (Parsons \& Cantlie 1948; Cantlie 1952). Dodona longicaudata has traditionally been listed as a subspecies of Dodona deodata Hewitson, 1876. However longicaudata has recently been reinstated 
as a distinct species based on the following diagnosis: "Both sexes have a narrower white band on both wings, reaching $3 \mathrm{~mm}$ on the male forewing and more infusion of the white areas with darker scaling. ... Male genitalia differs from $D$. deodata in the longer tegument, wider valvae, and a longer and squared saccus." (Callaghan 2009). Both deodata and longicaudata were given as subspecies of Dodona henrici, a Chinese species, by Evans (Evans 1932).

306. Zemeros flegyas flegyas Cramer, 1780 - Himalayan Punchinello: This species was very common in evergreen forests along Taidang and Jidung streams near Gongrot, and near Halwa Atong. Most of our sightings were from November 2009. A female was seen laying eggs on Maesa sp. (Myrsinaceae), and many caterpillars were subsequently discovered on this plant. The species is also common in the Khasi Hills (Parsons \& Cantlie 1948).

\section{DISCUSSION}

We presented above the first annotated checklist of butterflies of the Garo Hills, and a preliminary analysis of butterfly diversity of this neglected but notably biodiverse mountain range. Our observations show that the Garo Hills support a substantial number of butterfly species that are rare, endemic and/or legally protected in India under Schedule I and II of the Wildlife (Protection) Act, 1972 (Anonymous 1997). We have also reported several important range extensions, including those of Prothoe franck regalis and Elymnias peali, both of which are very rare and protected under Schedule I of the Wildlife (Protection) Act, 1972. These findings underscore the importance of the Garo Hills as an area of tremendous national and global conservation significance, especially with reference to butterflies. Moreover, the total number of butterfly species and the number of legally protected species recorded by us in the Garo Hills are likely to increase significantly as more systematic and long-term surveys are undertaken in the future. We hope that our preliminary data and analyses will form a baseline for butterfly population ecological and biodiversity studies in this region. The numbers reported here may also be useful in comparative studies in several decades as the Garo Hills landscape changes in face of ongoing human population expansion and associated growth of agriculture, mining and other developmental activities.

The known butterfly fauna of the Garo Hills compares with the butterfly fauna of neighboring Bangladesh (Larsen 2004). During his three years in Bangladesh, Larsen personally collected 236 species and noted 75 other confirmed records from published literature, reporting a total of 311 species for Bangladesh. He further listed 116 additional species as "certain to occur" in the country, with a minimum total of 427 butterfly species, and a "Likely total fauna 500-550" species for Bangladesh. We recorded 298 species from the Garo Hills during only 49 days of field work from four visits spread across two seasons. We suspect that the total butterfly fauna of the Garo Hills will be 600 to 650 species, broadly overlapping with but somewhat exceeding the Bangladesh butterfly fauna. This is because: (a) we have recorded many species not recorded by Larsen (2004), (b) some of the widespread and common species that Larsen reported for Bangladesh but we have not yet recorded in the Garo Hills are expected to be present there, and (c) our species accumulation curve was still climbing steeply at the end of our preliminary surveys. Moreover, many mid-elevation butterfly species are expected to be in the Nokrek NP in the Western Garo Hills District, but they will not occur in Bangladesh for lack of suitable altitudinal range. It is our aim to further explore Nokrek and other relatively neglected areas in the near future to document butterfly fauna in this region. When this is done, we expect the butterfly fauna of the Garo Hills to compare fairly closely with the butterfly fauna of the neighboring Khasi Hills, from which 600-650 species have so far been reported (Swinhoe 1893, 1896; Parsons \& Cantlie 1948; Evans 1949; Cantlie 1952, 1956). For this reason, we have compared in the annotated checklist above the occurrence and status of butterflies from the Khasi Hills with our observations in the Garo Hills. However, some high-elevation butterflies that occur in the Khasi Hills are unlikely to be present in the Garo Hills, whose average and maximum elevations are considerably lower than that of the Khasi Hills.

We recognize several limitations of our preliminary surveys. First, we have surveyed butterflies for a total of less than 50 days, which is not sufficient for such a biodiverse region. Second, our sampling was not uniform for all species groups and genera. 
Some genera were exceptionally well represented, e.g., Papilio, Graphium, Athyma, Charaxes and Euploea (Fig. 4), which are usually very large and conspicuously patterned and therefore easy to locate and identify. On the other hand, genera such as Neptis, Ypthima, Potanthus, Pelopidas and Caltoris were poorly represented. These genera are very well represented in the northeastern Indian butterfly fauna but their members are either inconspicuously patterned or very difficult to identify to sub/species level without dissecting male genitalia. We hope to obtain collecting permits and record species richness in these genera within the Garo Hills in the near future. Lastly, our sampling was not uniform across observers or seasons. Some of us quantified all our sightings whereas others collected only presence-absence data or recorded the number of butterflies only approximately. We hope to use a uniform sampling strategy for all future surveys in the Garo Hills and elsewhere in India. We sampled mostly during the pre-monsoon and postmonsoon months but not at all during the winter or spring, and therefore may have missed species that are more frequent in those seasons. For future studies it may also be advisable to use crab and other baits in a standardized manner by placing bait traps at regular intervals and running the bait traps across seasons and in different parts of the Garo Hills for similar durations. However, we recommend the "fixed time interval" sampling strategy used by KK and SS over conventional belt transects or Pollard walks because our counts seem to capture more species and cover butterfly habitats much better than the conventional methods. We will present a comparative analysis of various field methods to gather quantitative data in a separate paper when we have data from coming field seasons.

Of Meghalaya's three major hill ranges, the Khasi and Jaintia hills have faced ecological, environmental and biodiversity disaster in the past 150 years due to extensive logging, coal mining and agriculture. As a result, majority of forests and biodiversity in the Khasi and Jaintia hills has vanished. Thus, the Garo Hills remain the last stronghold of biodiversity in Meghalaya, and host the largest forested area in all of the Indian part of the Indo-Myanmar Biodiversity Hotspot. Unfortunately, jhum cultivation, monoculture plantations, rampant illegal coal mining, proposed uranium mining, hunting and poaching leading to habitat destruction and faunal depletion now threaten the flora and fauna of the Garo Hills on a large scale. Therefore, it is important to undertake intensive, long-term butterfly surveys in the Garo Hills in order to record the butterfly fauna while forests still exist in large tracts. Our work may contribute to butterfly faunal discovery, and it may also prove useful in longterm ecological and conservation studies. Moreover, we hope that the findings reported above will help forest officials and other state and national agencies concerned with forest and wildlife conservation in designing and implementing informed conservation action plans for the Garo Hills. Based on our observations, we suggest that it is very important to protect riparian forests and some large tracts of evergreen forests, where most of the butterfly species occur. This will ensure longterm persistence of majority of the butterfly fauna of the Garo Hills. Protection of smaller forest fragments spread across the landscape may also act as habitat islands that may facilitate movement of butterflies and other small-bodied organisms between larger forest patches in national parks and reserved forests. Such smaller forest fragments are not necessarily under the jurisdiction of the forest department, local communities often own such forest patches in the Garo Hills landscape. Forests owned by various akings in the Garo Hills, including the Matcha Nokpante Community Reserve, are good examples of such community-owned forest patches that are important butterfly habitats where we have seen several Schedule I and II species. A major challenge for the near future is to encourage the local tribal communities to continue their traditional protection of these forest patches in face of outside pressure from illegal coal miners. The Meghalaya Forest Department and Samrakshan Trust are involved in participatory management of these and other forests surrounding the Balpakram NP. We hope that our information will help them with this effort and help generate forest-based revenue for the Garo communities with initiates such as butterfly tourism. Such a bottom-up approach to forest and wildlife conservation may be especially necessary in Meghalaya where vast forested areas are owned not only by the forest department, but also by the Garo tribal communities. It is important to note that nearly half of the evergreen and semi-evergreen forests in the Balpakram-Baghmara landscape are on community land owned by various akings, and their combined 
importance for long-term butterfly conservation must be considerable, indeed.

\section{REFERENCES}

Anonymous (1997). The Wildlife (Protection) Act, 1972 (as amended up to 1993) with rules uptil 1995. Natraj Publishers, Dehra Dun.

Butler, A.G. (1879). On a collection of Lepidoptera from Cachar. Transactions of the Entomological Society of London 1879: 1-8.

Callaghan, C.J. (2009). The riodinid butterflies of Vietnam (Lepidoptera). Journal of the Lepidopterists' Society 63: 61-82.

Cantlie, K. (1952). More butterflies of the Khasi and Jaintia Hills, Assam. Journal of the Bombay Natural History Society 51: 42-60.

Cantlie, K. (1956). Hesperiidae of Khasi and Jaintea Hills. Journal of the Bombay Natural History Society 54: 212 215.

Champion, H.G. \& S.K. Seth (1968). A Revised Survey of the Forest Types of India. Manager of Publications, Delhi, 404pp.

Corbet, A.S., H.M. Pendlebury \& J.N. Eliot (1992). The Butterflies of the Malay Peninsula. 4th Revised Ed. Malayan Nature Society, Kuala Lumpur, 595pp.

d'Abrera, B. (1986). Butterflies of the Oriental Region. Part III: Lycaenidae and Riodinidae. Hill House, Melbourne, 536-672pp.

Evans, W.H. (1932). The Identification of Indian butterflies2nd Edition. Bombay Natural History Society, Mumbai, 454pp.

Evans, W.H. (1949). A Catalogue of the Hesperiidae from Europe, Asia and Australia in the British Museum (Natural History). British Museum (Natural History), London, $502 \mathrm{pp}$.

Forest Survey of India (2009). India State of Forest Report 2009. Ministry of Environment and Forests, Government of India, Dehradun, 199pp.

Gupta, I.J. \& D.K. Mondal (2005). Red Data Book (Part-2) - Butterflies of India. Zoological Survey of India, Kolkata, $535 \mathrm{pp}$.

Io, C. (ed.) (2000). Monograph of Chinese Butterflies (Revised Edition). Henan Scientific and Technological Publishing House, $845 \mathrm{pp}$.

Kunte, K. (2009). Occurrence of Elymnias obnubila Marshall and de Nicéville, 1883 (Lepidoptera: Nymphalidae: Satyrinae) in southern Mizoram: Range extension of the species and an addition to the Indian butterfly fauna. Journal of Threatened Taxa 1(11): 567-568.

Kunte, K. (2010). Rediscovery of the federally protected Scarce Jester Butterfly Symbrenthia silana de Nicéville, 1885 (Nymphalidae: Nymphalinae) from the Eastern Himalaya and Garo Hills, northeastern India. Journal of Threatened
Taxa 2(5): 858-866.

Kunte, K. (in prep.). A Systematic and Biogeographic Catalogue of Indian Butterflies: Butterfly Diversity and Endemism in India's biodiversity hotspots. Indian Foundation for Butterflies, Bengaluru.

Kunte, K., E. Kunhikrishnan, M. Balakrishnan \& C. Susanth. (2008). Status and distribution of Appias lalage butterfly (Lepidoptera: Pieridae) in the Western Ghats, south-western India. Journal of the Bombay Natural History Society 105: 354-357.

Kunte, K., S. Kalesh \& U. Kodandaramaiah (eds.). (2011). Butterflies of India. v. 1.03. Indian Foundation for Butterflies, Bengaluru. $<$ http://ifoundbutterflies.org/>, Accessed 2011.

Larsen, T.B. (2004). Butterflies of Bangladesh - An Annotated Checklist. IUCN Bangladesh Country Office, Dhaka, $147 \mathrm{pp}$.

Moore,F.(1890-1892). Lepidoptera Indica. Vol. I. Rhopalocera. Family Nymphalidae. Sub-families Euploeinae and Satyrinae. Reeve \& Co, London, 317pp.

Moore, F. (1893-1896). Lepidoptera Indica. Vol. II. Rhopalocera. Family Nymphalidae. Sub-families Satyrinae (continued), Elymniinae, Amathusiinae, Nymphalinae (group Charaxina). Reeve \& Co, London, 274pp.

Moore, F. (1896-1899). Lepidoptera Indica. Vol. III. Rhopalocera. Family Nymphalidae. Sub-families Nymphalinae (continued), groups Potamina, Euthaliina, Limenitina. Reeve \& Co, London, 254pp.

Moore, F. (1899-1900). Lepidoptera Indica. Vol. IV. Rhopalocera. Family Nymphalidae. Sub-families Nymphalinae (continued), groups Limenitina, Nymphalina, and Argynnina. Reeve \& Co, London, 260pp.

Moore,F.(1901-1903).Lepidoptera Indica. Vol. V.Rhopalocera. Family Nymphalidae. Sub-family Nymphalinae (continued), groups Melitaeina and Eurytelina. Sub-families Acraeinae, Pseudergolinae, Calinaginae, and Libytheinae. Family Riodinidae. Sub-family Nemeobiinae. Family Papilionidae. Sub-families Parnassiinae, Thaidinae, Leptocircinae, and Papilioninae. Reeve \& Co, London, 248pp.

Moore, F. (1903-1905). Lepidoptera Indica. Vol. VI. Rhopalocera. Family Papilionidae. Sub-family Papilioninae (continued). Family Pieridae. Sub-family Pierinae. Reeve \& Co, London, 240pp.

Nair, M. (2011). Three new butterfly records for peninsular India: Dusky Yellow-breasted Flat Gerosis phisara (Moore), Common Gem Poritia hewitsoni Moore and Great Nawab Polyura eudamippus (Doubleday) from Similipal hills, Odisha, India. Journal of Threatened Taxa 3(3): 16241628.

Parsons, R.E. \& K. Cantlie (1948). The butterflies of the Khasia and Jaintia hills, Assam. Journal of the Bombay Natural History Society 47: 498-522.

Pinratana, A. (1981). Butterflies in Thailand. Vol. 4: Lycaenidae. Brothers of St. Gabriel in Thailand, Bangkok, $215 \mathrm{pp}$.

Radhakrishnan, C., J.R.B. Alfred \& M.R. Rynth (1989). Butterflies of Shillong and Its Environs. Science \& 
Technology Cell, Govt of Meghalaya, Shillong, 70pp.

Swinhoe, C. (1893). A list of the Lepidoptera of the Khasia Hills. Part I. Transactions of the Entomological Society of London 1893(41): 267-330.

Swinhoe, C. (1896). New species of Lepidoptera from Khasia Hills. Annals and Magazine of Natural History, including Zoology, Botany, and Geology (6)17: $357-363$.

Swinhoe, C. (1905-1910). Lepidoptera Indica. Vol. VII. Rhopalocera. Family Pieridae [printed in error: "Family Papilionidae"]. Sub-family Pierinae (continued). Family Lycaenidae. Sub-families Gerydinae, Lycaenopsinae and Everinae. Reeve \& Co, London, 286pp.

Swinhoe, C. (1910-1911). Lepidoptera Indica. Vol. VIII. Rhopalocera. Family Lycaenidae. Sub-families Lycaeninae, Plebeinae, Lampidinae, Chrysophaninae, Poritiinae, Amblypodiinae, Curetinae, Liphyrinae, Ruralinae. Reeve \& Co, London, 293pp.

Swinhoe,C.(1911-1912).Lepidoptera Indica. Vol.IX.Rhopalocera. FamilyLycaenidae (continued). Sub-families Horaginae, Deudorixinae, Hypolycaeninae, Zesiusinae, Aphnaeinae, Biduandinae, Cheritrinae, Loxurinae. Family Hesperiidae. Subfamilies Ismeneinae, Achalarinae. Reeve \& Co, London, 278pp.

Swinhoe, C. (1912-1913). Lepidoptera Indica. Vol. X. Rhopalocera. Family Hesperiidae (concluded). Sub-families Celaenorrhinae, Hesperiinae, Pamphilinae, Astictopterinae, Suastinae, Erionotinae, Matapinae, Notocryptinae, Plastingiinae, Erynninae. Reeve \& Co, London, 364pp.

Talbot, G. (1947). The Fauna of British India, including Ceylon and Burma: Butterflies, Vol. 2. Taylor and Francis, London, 506pp.

Tytler, H.C. (1911). Notes on butterflies from the Naga Hills. Part I. Journal of the Bombay Natural History Society 21: 48-65.

Tytler, H.C. (1912). Notes on butterflies from the Naga Hills. Part II. Journal of the Bombay Natural History Society 21: 588-606.

Tytler, H.C. (1914). Notes on some new and interesting butterflies from Manipur and the Naga Hills. Part I. Journal of the Bombay Natural History Society 23: 216-229.

Tytler, H.C. (1915a). Notes on some new and interesting butterflies from Manipur and the Naga Hills. Part II. Journal of the Bombay Natural History Society 23: 502-515.

Tytler, H.C. (1915b). Notes on some new and interesting butterflies from Manipur and the Naga Hills. Part III. Journal of the Bombay Natural History Society 24: $119-155$.

Tytler, H.C. (1926a). Notes on some new and interesting butterflies from India and Burma. Part I. Journal of the Bombay Natural History Society 31: 248-260.

Tytler, H.C. (1926b). Notes on some new and interesting butterflies from India and Burma. Part II. Journal of the Bombay Natural History Society 31: 579-590.

Wynter-Blyth, M.A. (1957). Butterflies of the Indian Region. Bombay Natural History Society, Mumbai, 523pp.
Author Details: Krushnamegh Kunte, PhD, is a Reader and Ramanujan Fellow at the National Center for Biological Sciences, Bengaluru, India. $\mathrm{He}$ is interested in studying the organization and evolution of biodiversity especially in the Indian Region, with a special focus on the ecology, evolution and genetics of morphological diversification and speciation in butterflies. Lab website: http://biodiversitylab.org.

SANJAY SONDHI is a Dehradun-based naturalist with an interest in nature conservation and environment protection. He is mainly interested in birds, butterflies and herpetofauna, and is the author of several books on these animals. His work includes faunal surveys, conservation education, protected areas management plans, and man-animal conflicts, and his special interests are writing and photography.

BENSEN M. SANGma is a native Garo who worked with Samrakshan Trust for many years to map the human-dominated forest mosaics in the Garo Hills and to survey elephant populations in this landscape. Now he oversees the nature- and tribal people-oriented eco-tourism initiatives in the Gongrot area with Samrakshan Trust.

ROHAN LOVALEKAR is a Chiplun(Maharashtra)based naturalist working in the banking sector. He spends his spare time surveying and monitoring butterflies in the northern Western Ghats. He has especially been studying the species richness and seasonal occurrences, and swarming and migratory behaviors of various butterflies. He also volunteers for the local nature-related NGO, Sahyadri Nisarga Mitra.

KEDAR TOKEKAR is a Pune(Maharashtra)-based naturalist who has traveled extensively in the Western and Eastern Himalaya to document species diversity of these biodiversity hotspots. Gaurav Agaverar is a Project Trainee at the National Center for Biological Sciences, Bengaluru, India. His special interests include genetics, butterflies, natural history, and photography.

Author Contributions: $\mathrm{KK}$ initiated and organized this research, performed background searches, confirmed all the identifications and wrote the paper with contributions from all other authors; KK, SS and KT collated field data and prepared figures and tables. GA prepared color plates. All authors contributed substantial species records.

Acknowledgements: We thank Kamal Medhi, Yaranajit Deka, Anirban Datta Roy and Arpan Sharma of Samrakshan Trust, and Firoz Ahmed of Aaranyak, for logistical support on our field trips. Thanks are also due to Pawan Kumar Agrahari (Divisional Forest Officer, Balpakram NP Division, Meghalaya Forests and Environment Department) for his help with these surveys. Deepa Agashe, Saloni Bhatia, Bana and Sumitra Brahma, Yaranajit Deka, Shailesh Deshpande, Kamal Medhi and John Shira assisted in the field, Amol Patwardhan provided photographs of Graphium cloanthus and Sumalia daraxa, Rhucha Vatturkar (NCBS) assisted in preparing the final manuscript, and Ginseng Sangma (Samrakshan Trust) translated the abstract in the Garo language. Photograph of Losaria coon was taken in the Museum of Comparative Zoology, Harvard University, USA, and is copyright of President and Fellows of Harvard College. This work was financially supported by the Indian Foundation for Butterflies, Titli Trust, and Samrakshan Trust. This is Publication No. 3 of the Indian Foundation for Butterflies (http:// ifoundbutterflies.org/bibliography/publicationsof-the-indian-foundation-for-butterflies). 